Marukami, J.

Osaka J. Math.

26 (1989), 1-55

\title{
THE PARALLEL VERSION OF POLYNOMIAL INVARIANTS OF LINKS
}

Dedicated to Professor Nagayoshi Iwahori on his sixtieth birthday

\author{
JUN MURAKAMI
}

(Received April 10, 1987)

\section{Introduction}

Let $\mathcal{L}$ be the set of link isotopy types and $X: \mathcal{L} \rightarrow \boldsymbol{C}$ a $\boldsymbol{C}$-valued invariant of links. For any positive integer $r$, one can define the $r$-parallel version $X^{(r)}$ : $\mathcal{L} \rightarrow \boldsymbol{C}$ of $X$ by putting

$$
X^{(r)}(K)=X\left(K^{(r)}\right), \quad K \in \mathcal{L},
$$

where $K^{(r)}$ is the $r$-parallel link of $K$ obtained by applying the operation $\phi^{(r)}$ (see Figure 1) to each crossing point of $K$.

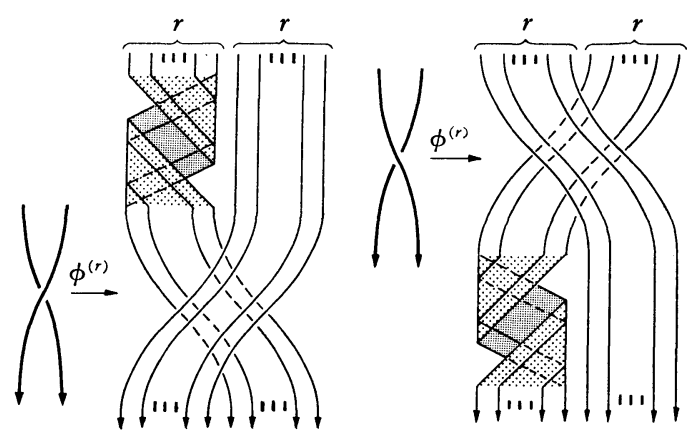

Figure 1

Recently, Morton-Short [24] and Yamada [34] independently noticed the existence of a pair of knots which is indistinguishable by the Jones polynomial [14], but is distinguishable by its 2-parallel version. Moreover there exist mutant knots distinguishable by the 3-parallel version of the two-variable Jones polynomial $P^{(3)}$ ([25] and Section 6.2 of this paper). Thus it seems to be worth while studying the $r$-parallel version of a link invariant from a general

* This research was supported in part by Grant-in-Aid for Scientific Research, The Ministry of Education, Science and Culture. 
view-point.

Throughout this paper we assume that all the invariants of links are of 'trace' type. A link invariant $X$ is called of trace type iff $X$ satisfies the following conditions: (i) Let $b^{\wedge}$ be the closure of an $n$-braid $b$. Then $X\left(b^{\wedge}\right)$ can be written as a linear combination of characters of representations of the braid group $B_{n}$. (ii) The characters in (i) satisfy some compatibility conditions with respect to the natural inclusion $B_{n} \rightarrow B_{n+1}(n=1,2, \cdots)$ (see Definition 1.1.4). For example, the (two-variable) Jones polynomial [7], [14], [29] and the Kauffman polynomial [17], [22] fulfill this assumption. (See also [2], [10], [15], [26].) The purpose of this paper is to give an efficient method to calculate the $r$-parallel version $X^{(r)}$ of the invariant $X$. A direct calculation of $X^{(r)}(K)$ is, in principle, possible, but the degrees of the characters of the representations involved soon become enormous even for relatively small $r$. We show that the characters can be reduced to a sum of those of much smaller degrees, and we have only to deal with representation matrices of much smaller sizes. See Table 1.

Table 1 The maximal degree of characters needed to get the $r$-parallel version of the Jones polynomial of the closures of $n$-braids.

\begin{tabular}{|c|c|c|c|c|c|c|c|c|c|c|}
\hline \multicolumn{2}{|c|}{$\begin{array}{l}\text { index } \\
\text { braid }\end{array}$} & 1 & 2 & 3 & 4 & 5 & 6 & 7 & 8 & 9 \\
\hline \multirow{2}{*}{3} & $\begin{array}{l}\text { direct } \\
\text { method }\end{array}$ & 2 & 9 & 48 & 297 & 2002 & 13260 & 90440 & 653752 & 4601610 \\
\hline & $\begin{array}{c}\text { our } \\
\text { method }\end{array}$ & 2 & 3 & 4 & 5 & 6 & 7 & 8 & 9 & 10 \\
\hline \multirow{2}{*}{4} & $\begin{array}{l}\text { direct } \\
\text { method }\end{array}$ & 3 & 28 & 297 & 3640 & 48450 & 653752 & 7020405 & 124062000 & 1739969550 \\
\hline & $\begin{array}{c}\text { our } \\
\text { method }\end{array}$ & 3 & 6 & 10 & 14 & 18 & 22 & 27 & 32 & 38 \\
\hline
\end{tabular}

One of the main results of this paper is the following, which is an immediate consequence of Thoerems 1.4.9, 1.5.1, 4.4.1, Corollary 4.2.3 and (4.1.10).

Theorem. Let $V$ be the one-variable Jones polynomial, $r$ a positive integer and $b$ a 3-braid whose closure is a knot. Then we have the following.

$$
V^{(r)}\left(b^{\wedge}\right)=\sum_{j=0}^{[r / 2]} c_{r, j}\left(\sum_{i=0}^{[3(r-j) / 2]} a_{3(r-j), i}(V) \operatorname{Trace}\left(\pi_{3(r-j), i}(b)\right),\right.
$$

where

$$
\begin{gathered}
c_{r, j}=\frac{r-2 j+1}{r+1}\left(\begin{array}{c}
r+1 \\
j
\end{array}\right), \\
a_{k, i}(V)=(-1)^{k+1} \frac{t^{k / 2+1 / 2-i}-t^{-k / 2-1 / 2+i}}{t-t^{-1}},
\end{gathered}
$$

and $\pi_{3 r, i}$ 's, are representations of $B_{3}$ given in Theorem 4.4.1.

The degree of the representation $\pi_{3 r, i}$ is equal to $i+1$ if $0 \leq i \leq r$ and 
$3 r-2 i+1$ if otherwise. Applications of the above theorem are given in (4.4.4)(4.4.10).

In the last section of this paper, we shall give a necessary condition for the existence of mutant knots distinguishable by the $r$-parallel version $X^{(r)}$ of the link invariant $X$ (Theorem 6.2.4). We construct mutant knots $K_{1}, K_{2}, K_{3}, K_{4}$ for which the 3 -parallel version $P^{(3)}\left(K_{i}\right)(1 \leq i \leq 4)$ of the two-variable Jones polynomial are all distinct (Section 6.2).

We shall give a formula for an invariant of cable links. Let $X$ be a link invariant of trace type. Then we shall construct several link invariants $X^{(r, 1)}$, $X^{(r, 2)}, \cdots$ by 'decomposing' the $r$-parallel version $X^{(r)}$ of $X$ (Theorems 1.5 .1 and 2.2.1). Let $K$ be a knot, $L$ a link in the solid torus and $K_{L}$ the satellite link [4] coming from $K$ and $L$. Let $X_{L}$ be a knot invariant denfied by $X_{L}(K)=$ $X\left(K_{L}\right)$. Then $X_{L}$ can be written as a linear combinations of invariants $X^{(r, 1)}$, $X^{(r, 2)}, \cdots$ if $L$ is a 'cable' (Definition 1.6.3), or if $X$ is the Jones polynomial or the Kauffman polynomial (Theorem 1.6.4, 2.2.1, 4.3.2, 5.3.1).

We prove, in Sections 1-2, fundamental formulas for $X^{(r)}$ and show some related results of general nature. In Sections 3-5 we apply the results of Sections 1-2 to the $r$-parallel version of the (two-avriable) Jones polynomial and the Kauffman polynomial, and investigate them more closely. It is discussed in Section 6 how $X^{(r)}$ works at mutant knots.

As a conclusion of this paper, the $r$-parallel version of link invariants seems to be quite promising in attacking the classification problem of link types.

Acknowledgement. I would like to thank S. Yamada for the useful discussions including the results of [34], which is the motivation of this study. I would like to express my appreciation to N. Iwahori, who suggested that the matrix entries given in Theorem 3.3.1 might be expressed by Gauss' polynomial, and to T. Kanenobu, who informed me about pairs of closed 3-braids with the same Kauffman polynomial. I am profoundly indebted to A. Gyoja, who gave me a lot of useful information about the Iwahori algebras (or Hecke algebras), for example, the result of [10]. I am also indebted to T. Kobayashi, who introduced me to the theory of the (two-variable) Jones polynomial. I am very grateful to N. Kawanaka, H. Nagao, K. Nagatomo, M. Nakaoka and M. Ochiai for their useful advice and kind encouragement. Last of all, I must thank muSIMP/muMATH (Symbolic Mathematics Package), TURBO Pascal for MSDOS on the personal computer PC-9801 (NEC) and AOS/VS Pascal on Eclipse MV/2000DC (Deta General), which are used for the calculations in the proof of Proposition 4.4.10 and in Example 6.2.7.

1. The parallel version of link invariants, I (the case of knots). We shall discuss in this and next sections general properties of a 'parallel ver- 
sion' of a link invariant of 'trace' type. In this section we first state and prove the results in the case of knots, and, in Section 2, generalize them to the case of links.

1.1. Link invariants of trace type. Let $B_{n}$ be Artin's braid group on $n$-strings with standard generators $\sigma_{1}, \sigma_{2}, \cdots, \sigma_{n-1}$ as in Figure 2,

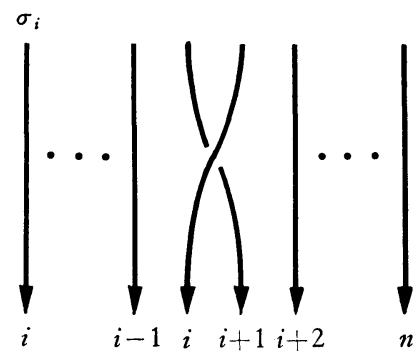

Figure 2

i.e.

$$
\begin{array}{r}
B_{n}=\left\langle\sigma_{1}, \sigma_{2}, \cdots, \sigma_{n-1}\right| \sigma_{i} \sigma_{i+1} \sigma_{i} \sigma_{i+1} \sigma_{i} \sigma_{i+1}(1 \leq i \leq n-2), \\
\left.\sigma_{i} \sigma_{j}=\sigma_{j} \sigma_{i}(1 \leq i<j-1 \leq n-2)\right\rangle .
\end{array}
$$

Let $\xi_{n}^{(r)}: B_{n} \rightarrow B_{n+r}$ be the group homomorphism defined by $\xi_{n}^{(r)}\left(\sigma_{i}\right)=\sigma_{i}$ for $n, r \in \boldsymbol{N}=\{1,2, \cdots\}$ and $1 \leq i \leq n-1$. We regard $B_{n}$ as a subgroup of $B_{n+}$, with respect to the inclusion $\xi_{n}^{(r)}$. Let $B=\left\{(b, n) \mid b \in B_{n}\right.$ for $\left.n=1,2, \cdots\right\}$. A closure of a braid $(b, n)$, written $(b, n)^{\wedge}$ or simply $b^{\wedge}$, is the link formed by joining the $n$ points at the top of $(b, n)$ to those at the bottom without further crossings. Two links $K_{1}, K_{2}$ are called equivalent if $K_{1}$ is ambient isotopic to $K_{2}$ in $\boldsymbol{R}^{3}$ ([3], Chapt. 1, B).

Theorem 1.1.1 (Alexander [1] p. 42). Every link is equivalent to the closure of a braid.

Definition 1.1.2 (Markov class). Let $\sim$ be the equivalence relation on $B$ generated by the following:

(i) $\left(b b^{\prime}, n\right) \sim\left(b^{\prime} b, n\right)$ for $b, b^{\prime} \in B_{n}$,

(ii) $(b, n) \sim\left(\sigma b_{u}^{ \pm 1}, n+1\right)$ for $b \in B_{n}$.

The equivalence classes of $B$ by the above relation are called Markov classes.

Theorem 1.1.3 (Markov [1] p. 51). The closures of two braids $b_{1}, b_{2}$ are equivalent if and only if $b_{1}$ and $b_{2}$ are contained in the same Markov class.

Definition 1.1 .4 (link invariant). A mapping $X$ from $B$ to a set $S$ is called a link invariant if $X$ is constant on each Markov class of $B$. 
Let $\boldsymbol{C}$ be the field of complex numbers and $B_{n}^{\wedge}$ the set of equivalence classes of finite dimensional irreducible representations of $B_{n}$ over $\boldsymbol{C}$. Let $f$ be a $\boldsymbol{C}$-valued function on $B$. We assume that $f$ can be written as a finite linear combination of characters of the braid group, i.e. for $b \in B_{n}$,

$$
f(b, n)=\sum_{\mu \in B_{\hat{n}}} a_{\mu}(f) \chi_{\mu}(b),
$$

where, for each $\mu \in B_{n}^{\wedge}, \chi_{\mu}$ is the corresponding character and $a_{\mu}(f)$ is an element of $\boldsymbol{C}$ independent of $b$. Let $\left(\rho_{\mu}, V_{\mu}\right)$ be the representation of $B_{n}$ corresponding to $\mu \in B_{n}^{\widehat{n}}$, i.e. $V_{\mu}$ is the representation space and $\rho_{\mu}$ is the group homomorphism of $B_{n}$ to $G L\left(V_{\mu}\right)$. Then $\rho_{\mu}$ is extended uniquely to a $C$-algebra homomorphism of the group ring $\boldsymbol{C} B_{n}$ to $\operatorname{End}\left(V_{\mu}\right)$ and so its character $\chi_{\mu}$ is extended to a linear function on $\boldsymbol{C} B_{n}$, which are also denoted by $\rho_{\mu}$ and $\chi_{\mu}$ respectively. Hence the function $f$ is extended to a function on $\boldsymbol{C} B=\{(b, n) \mid b \in$ $C B_{n}$ for $n \in N$ \} by (1.1.5), which is also denoted by $f$.

Definition 1.1.6 (associated algebra). Let $A_{n}(f)^{\wedge}=\left\{\mu \in B_{n}^{\wedge} \mid a_{\mu}(f) \neq 0\right\}$ for $n \in \boldsymbol{N}$ and $A_{n}(f)=\underset{\mu_{\in} A_{n}(f) \wedge}{\rho_{\mu}}\left(\boldsymbol{C} B_{n}\right)$. We call $A_{n}(f)(n=1,2, \cdots)$ the associated algebra of $f$. Let $p_{n}: \boldsymbol{C} B_{n} \rightarrow A_{n}(f)$ be the algebra homomorphism defined by $p_{n}=\underset{\mu \in A_{n}(f) \wedge}{\bigoplus_{\mu}} \rho_{\mu}$.

Definition 1.1.7 (trace type). A $\boldsymbol{C}$-valued function $f$ on $B$ is called of trace type if $f$ satisfies the following conditions:

(i) The function $f$ can be written as a linear combination of characters of $B_{n}$ as in (1.1.5) for euch $n \in \boldsymbol{N}$.

(ii) Let $A_{1}(f), A_{2}(f), \cdots$ be the associated algebras of $f$. Then there are algebra homomorphisms $\zeta_{n}: A_{n}(f) \rightarrow A_{n+1}(f)$ for which ${p_{n+1}}^{\circ} \xi_{n}^{(1)}=$ $\xi_{n} \circ p_{n}$.

Remark 1.1.8. Both the one (two)-variable Jones polynomial and the Kauffman polynomial are of trace type for the generic values of their parameter(s).

To specify the notations, we review the definitions of the polynomial invariants of links mentioned in Remark 1.1.8. We also recall the definitions of the regular isotopy invariants of unoriented link diagrams called the bracket polynomial and the $L$-polynomial [16], [17] which will be needed in Sections 4 and 5.

Definition 1.1.9 (writhe). The writhe of a link diagram $K$ is the sum of the signatures of all crossing points of $K$ (Figure 3 ) and is denoted by $w(K)$.

Definition 1.1.10. Let $t$ be a non-zero complex number. The bracket polynomial $\langle\cdot\rangle=\langle\cdot\rangle(t)$ with values in $\boldsymbol{C}$ is uniquely defined for regular isotopy classes of the unoriented link diagrams by the following formulas: 


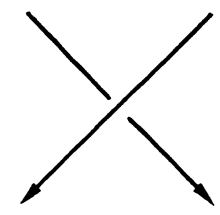

positive crossing signature $=1$

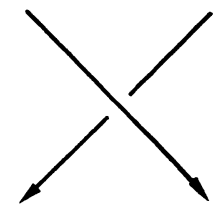

negative crossing signature $=-1$

Figure 3

$$
\left\langle K_{+}\right\rangle=t^{-1 / 4}\left\langle K_{0}\right\rangle+t^{1 / 4}\left\langle K_{\infty}\right\rangle, \quad\langle O\rangle=1 \text { for the unknot } O,
$$

where the $K_{*}$ are identical except within a ball where they are as in Figure 4.

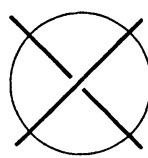

$K_{+}$

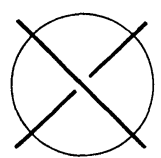

K.

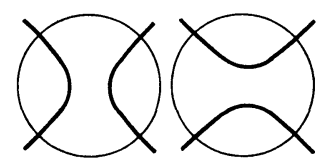

$K_{\text {r }}$

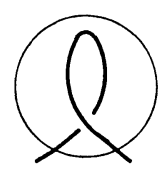

$K_{l+}$

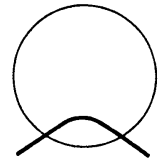

$K_{\text {n }}$

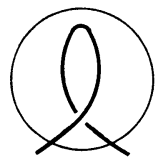

$K_{l-}$

Figure 4

Let $a$ and $x$ be non-zero complex numbers. The $D$-polynomial $D(\cdot)=$ $D(\cdot)(a, x)$ with values in $\boldsymbol{C}$ is uniquely defined for regular isotopy classes of the unoriented link diagrams by the following formulas:

$$
\begin{aligned}
& D\left(K_{+}\right)-D\left(K_{-}\right)=x\left(D\left(K_{0}\right)-D\left(K_{\infty}\right)\right), \\
& D\left(K_{l^{+}}\right)=a D\left(K_{n}\right), \quad D\left(K_{l^{-}}\right)=a^{-1} D\left(K_{n}\right), \quad D(O)=1,
\end{aligned}
$$

where the $K_{*}$ are identical except within a ball where they are as in Figure 4 .

In the following, we define isotopy invariants of oriented links. Let $t$ be a non-zero complex number. The one-variable Jones polynomial $V(\cdot)=V(\cdot)(t)$ with values in $\boldsymbol{C}$ is defined by

$$
t^{-1} V\left(K_{+}\right)-t V\left(K_{-}\right)=\left(t^{1 / 2}-t^{-1 / 2}\right) V\left(K_{0}\right), \quad V(O)=1,
$$

where the $K_{*}$ are identical except within a ball where they are as in Figure 5 . To specify the parameter $t$, we also denote $V(K)$ by $V(K)(t)$ for a link $K$. Let $K$ be a link diagram and $|K|$ its unoriented diagram. Then it is known [16] that

$$
V(K)(t)=\left(-t^{3 / 4}\right)^{w(K)}\langle|K|\rangle(t) .
$$




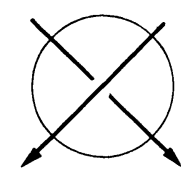

$K_{+}$

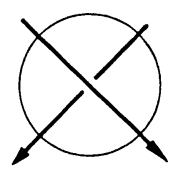

K.

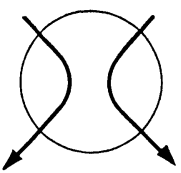

$K_{0}$

Figure 5

Let $l$ and $m$ be non-zreo complex numbers. The two-variable Jones polynomial $P(\cdot)=P(\cdot)(l, m)$ with values in $C$ is defined by

$$
l^{-1} P\left(K_{+}\right)+l P\left(K_{-}\right)+m P\left(K_{0}\right)=0, \quad P(O)=1,
$$

where we use the notation in (1.1.11).

Let $a$ and $x$ be non-zero complex numbers and $K$ be a link diagram. The Kauffman polynomial $F(\cdot)=F(\cdot)(a, x)$ with values in $C$ is defined by $F(K)=a^{-w(K)} D(|K|)$.

1.2. Parallel versions. Fix a positive integer $r$. Let $\phi_{n}^{(r)}: B_{n} \rightarrow B_{r n}$ be the group homomorphism defined by Figure 1, or, equivalently, by

$$
\begin{gathered}
\phi_{n}^{(r)}\left(\sigma_{i}\right)=\sigma(r i-r+1, r i-1)^{-r} \sigma(r i, r i+r-1) \sigma(r i-1, r i+r-2) \cdots \\
\sigma(r i-r+1, r i) \quad(1 \leq i \leq n-1),
\end{gathered}
$$

where $\sigma(i, j)=\sigma_{i} \sigma_{i+1} \cdots \sigma_{j}$. Let $\phi^{(r)}: B \rightarrow B$ be the map defined by $\phi^{(r)}((b, n))=$ $\left(\phi_{n}^{(r)}(b), r n\right)$.

Theorem 1.2.2. Let $\left(b_{1}, n_{1}\right)$ and $\left(b_{2}, n_{2}\right)$ be two braids. Then the links $\left(\phi_{n_{1}}^{(r)}\left(b_{1}\right), r n_{1}\right)^{\wedge}$ and $\left(\phi_{n_{2}}^{(r)}\left(b_{2}\right), r n_{2}\right)^{\wedge}$ are equivalent if $\left(b_{1}, n_{1}\right)^{\wedge}$ and $\left(b_{2}, n_{2}\right)^{\wedge}$ are equivalent.

The following lemma and Theorem 1.1.3 yield the above theorem.

Lemma 1.2.3. Let $\sim$ denote the Markov equivalence relation (Definition 1.1.2). Then we have the following :

(a) For $b_{1}, b_{2} \in B_{n},\left(\phi_{n}^{(r)}\left(b_{1} b_{2}\right), r n\right) \sim\left(\phi_{n}^{(r)}\left(b_{2} b_{1}\right), r n\right)$,

(b) For $b \in B_{n},\left(\phi_{n}^{(r)}(b), r n\right) \sim\left(\phi_{n+1}^{(r)}\left(b \sigma_{n}^{ \pm 1}\right), r n+r\right)$.

Proof. The part (a) holds since $\phi_{n}^{(r)}$ is a group homomorphism. The following Lemma 1.2.4 shows the part (b).

Lemma 1.2.4. For $b \in B_{n}(n \geq r)$,

$$
\begin{aligned}
& (b, n) \sim\left(b \sigma_{n}^{(r)}, n+r\right), \\
& (b, n) \sim\left(b\left(\sigma_{n}^{(r)}\right)^{-1}, n+r\right) .
\end{aligned}
$$

where $\sigma_{n}^{(r)}=\sigma(n-r+1, n-1)^{-r} \sigma(n, n+r-1) \sigma(n-1, n+r-2) \cdots \sigma(n-r+1, r i)$. 
Proof. We first prove (1.2.5) by induction on $r$. For $r=1,(1.2 .5)$ is identical to Definition 1.1.2 (ii). Suppose that (1.2.5) is true for $r=k-1$. By Definition 1.1.2 (i) and (ii) we have

$$
\begin{aligned}
& \left(b \sigma_{n}^{(k)}, n+k\right) \\
& \sim\left(\sigma(n-1, n+k-2) \cdots \sigma(n-k+1, n) b \sigma(n-k+1, n-1)^{-k}\right. \\
& \left.\quad \times \sigma(n, n+k-2) \sigma_{n+k-1}, n+k\right) \\
& \sim\left(\sigma(n-1, n+k-2) \cdots \sigma(n-k+1, n) b \sigma(n-k+1, n-1)^{-k}\right. \\
& \quad \times \sigma(n, n+k-2), n+k-1) \\
& \sim\left(b \sigma(n-k+1, n-1)^{-k} \sigma(n, n+k-2) \sigma(n-1, n+k-2) \cdots\right. \\
& \quad \sigma(n-k+1, n), n+k-1) .
\end{aligned}
$$

By using the relation of the braid group, we have $\sigma_{k}^{-1} \sigma(i, j)^{-1}=\sigma(i, j)^{-1} \sigma_{k+1}^{-1}$, and so we get

$$
\begin{aligned}
& \sigma(n-k+1, n-1)^{-k+1} \\
& \quad=\sigma(n-k+2, n-1)^{-1} \sigma_{n-k+1}^{-1} \sigma(n-k+1, n-1)^{-k+2} \\
& \quad=\sigma(n-k+2, n-1)^{-1} \sigma(n-k+1, n-1)^{-k+2} \sigma_{n-1}^{-1} \\
& \quad=\sigma(n-k+2, n-1)^{-2} \sigma_{n-k+1}^{-1} \sigma(n-k+1, n-1)^{-k+3} \sigma_{n-1}^{-1} \\
& \quad=\sigma(n-k+2, n-1)^{-2} \sigma(n-k+1, n-1)^{-k+3} \sigma_{n-2}^{-1} \sigma_{u-1}^{-1} \\
& \quad=\cdots=\sigma(n-k+2, n-1)^{-k+1} \sigma_{n-k+1}^{-1} \sigma_{u-k+2}^{-1} \cdots \sigma_{n-1}^{-1} .
\end{aligned}
$$

By using the relation of the braid group, we have $\sigma(i, j) \sigma(i-1, j)=$ $\sigma(i-1, j) \sigma(i-1, j-1)=\sigma_{i-1} \sigma(i, j) \sigma(i-1, j-1)$. Hence we get

(1.2.9) $\sigma(n, n+k-2) \sigma(n-1, n+k-2) \sigma(n-2, n+k-3) \cdots \sigma(n-k+1, n)$

$$
\begin{aligned}
= & \sigma_{n-1} \sigma(n, n+k-2) \sigma(n-1, n+k-3) \sigma(n-2, n+k-3) \cdots \\
& \sigma(n-k+1, n) \\
= & \sigma_{n-1} \sigma_{n-2} \sigma(n, n+k-2) \cdots \sigma(n-2, n+k-4) \sigma(n-3, n+k-4) \cdots \\
& \sigma(n-k+1, n) \\
= & \cdots=\sigma_{n-1} \sigma_{n-2} \cdots \sigma_{n-k+1} \sigma(n, n+k-2) \cdots \sigma(n-k+1, n-1) .
\end{aligned}
$$

By substituting (1.2.8) and (1.2.9) into (1.2.7), we obtain

$$
\begin{aligned}
\left(b \sigma_{n}^{(r)}, n+k\right) & \\
\sim & \left(b \sigma(n-k+1, n-1)^{-k} \sigma(n, n+k-2) \sigma(n-1, n+k-2) \cdots\right. \\
& \sigma(n-k+1, n), n+k-1) \\
= & \left(b \sigma(n-k+1, n-1)^{-1} \sigma(n-k+2, n-1)^{-k+1} \sigma_{n-k+1}^{-1} \sigma_{n-k+2}^{-1} \cdots\right. \\
& \sigma_{n-1}^{-1} \sigma_{n-1} \sigma_{n-2} \cdots \sigma_{n-k+1} \sigma(n, n+k-2) \cdots \\
& \sigma(n-k+2, n-1) \sigma(n-k+1, n-2), n+k-1)
\end{aligned}
$$




$$
\begin{aligned}
= & \left(b \sigma(n-k+1, n-1)^{-1} \sigma(n-k+2, n-1)^{-k+1} \sigma(n, n+k-2) \cdots\right. \\
& \sigma(n-k+2, n-1) \sigma(n-k+1, n-1), n+k-1) \\
= & \left(b \sigma(n-k+1, n-1)^{-1} \sigma_{n}^{(k-1)} \sigma(n-k+1, n-1), n+k-1\right) .
\end{aligned}
$$

Moreover, Lemma 1.2.3 (a) and the induction hypothesis imply that

$$
\left(b \sigma(n-k+1, n-1)^{-1} \sigma_{n}^{(k-1)} \sigma(n-k+1, n-1), n+k-1\right) \sim(b, n) .
$$

Hence (1.2.5) is proved. An analogous argument yields (1.2.6).

Definition 1.2.10 ( $r$-parallel version). Let $K$ be a link and $(b, n) \in B$ the braid whose closure is equivalent to $K$. The link $\left(\phi_{n}^{(r)}(b), n\right)^{\wedge}$ is called the $r$ parallel version of $K$ and denoted by $K^{(r)}$. The $r$-parallel version $f^{(r)}$ of a function $f$ on $B$ is defined by $f^{(r)}(b, n)=f\left(\phi_{n}^{(r)}(b), r n\right)$.

The $r$-parallel version of a link is well-defined by Theorem 1.2.2. Hence the parallel version $X^{(r)}$ of a link invariant $X$ is again a link invariant.

1.3. Wreath products. We review representation theory of wreath products. The references are [19] and [6]. Throughout this paper, a semigroup has a unit, a semigroup homomorphism preserves the unit and a linear representation of a group or a semigroup sends the unit to the identity transformation. Let $\Omega=\{1,2, \cdots, n\}$ and $H$ a semigroup with a semigroup homomorphism $\theta: H \rightarrow S_{n}$, where $S_{n}$ denotes the symmetric group of degree $n$ actnig on $\Omega$ naturally. For $h \in H$, let $\langle\theta(h)\rangle$ denote the subgroup of $S_{n}$ generated by $\theta(h)$. Fix a group $G$ and an element $h_{0}$ of $H$.

$$
H\left(h_{0}\right)=\left\{h \in H \mid \text { for every }\left\langle\theta\left(h_{0}\right)\right\rangle \text {-orbit } O \text { of } \Omega, \theta(h) \cdot O=O\right\},
$$

which is a subsemigroup of $H$. The semigroup $H\left(h_{0}\right)$ acts on $G^{n}$ by the following. For $h \in H\left(h_{0}\right)$ and $g=\left(g_{1}, g_{2}, \cdots, g_{n}\right) \in G^{n},{ }^{h} g=\left(g_{\theta(h)^{-1}(1)}, \cdots, g_{\theta(h)^{-1}(n)}\right)$.

Definition 1.3.2. The set $G^{n} \times H\left(h_{0}\right)$ together with the composition law $(g, h)\left(g^{\prime}, h^{\prime}\right)=\left(g\left({ }^{h} g^{\prime}\right), h h^{\prime}\right)$ is a semigroup called the wreath product $G^{n} \backslash H\left(h_{0}\right)$ of $G$ with $H\left(h_{0}\right)$.

For a group $G$ let $G^{\wedge}$ denote the set of equivalence classes of finite dimensional irreducible representations of $G$ over $\boldsymbol{C}$, and for $\nu \in G^{\wedge},\left(\rho_{\nu}, V_{\nu}\right)$ denotes the corresponding representation. For $\nu=\left(\nu_{1}, \cdots, \nu_{n}\right)\left(\nu_{j} \in G^{\wedge}\right)$, let $\rho_{\nu}=\rho_{\nu_{1}} \otimes \cdots \otimes \rho_{\nu_{n}}$, which is an element of $\left(G^{n}\right)^{\wedge}$. In the following, we assume that $\nu_{i}=\nu_{j}$ if $\left\langle\theta\left(h_{0}\right)\right\rangle \cdot i=\left\langle\theta\left(h_{0}\right)\right\rangle \cdot j$. We define an action of the semi-group $G^{n} \rtimes H\left(h_{0}\right)$ on $V_{\nu}$ by the following: For $(g, h) \in G^{n} \rtimes H\left(h_{0}\right)$ and $\left(v_{1}, \cdots, v_{n}\right)$ $\in V_{\nu_{1}} \otimes \cdots \otimes V_{\nu_{n}}$

$$
(g, h)\left(v_{1}, \cdots, v_{n}\right)=\rho_{\nu}(g)\left(v_{\theta(h)^{-1}(1)}, \cdots, v_{\theta(h)^{-1}(n)}\right) .
$$


We denote by $\rho_{\nu} \sim$ the representation of the semigroup $G^{n} \rtimes H\left(h_{0}\right)$ given by the above action on $V_{\nu}$. If $\rho_{\nu}(g)=\left(\rho_{\nu_{1}}\left(g_{1}\right)_{\alpha_{1} \beta_{1}} \cdots \rho_{\nu_{n}}\left(g_{n}\right)_{\alpha_{n} \beta_{n}}\right)\left(1 \leq \alpha_{1}, \beta_{1} \leq \operatorname{dim} V_{\nu_{1}}, \cdots, 1\right.$ $\left.\leq \alpha_{n}, \beta_{n} \leq \operatorname{dim} V_{v_{n}}\right)$ is a matrix representing $g=\left(g_{1}, \cdots, g_{n}\right) \in G^{n}$, then we get for $h \in H\left(h_{0}\right)$ :

$$
\rho_{\nu} \sim(g, h)=\left(\rho_{\nu_{1}}(g)_{1 \alpha_{1} \beta_{\theta(h)^{-1}(1)}} \cdots \rho_{\nu_{n}}\left(g_{n}\right)_{\left.\alpha_{n} \beta_{\theta(n)}\right)^{-1}(h)}\right) .
$$

Let $\pi$ be a representation of the semigroup $H\left(h_{0}\right)$. By composing the canonical projection $G^{n} \searrow H\left(h_{0}\right) \rightarrow H\left(h_{0}\right), \pi$ is naturally extended to a representation of the semigroup $G^{n} \searrow H\left(h_{0}\right)$, which will also be denoted by $\pi$.

Proposition 1.3.4. Let $(\rho, V)$ be a finite dimensional linear representation of the semigroup $G^{n} \mathrm{X} H\left(h_{0}\right)$ such that the restriction $\left.\rho\right|_{G^{n}}$ is a completely reducible group representation. We assume that the irreducible components of $\left.\rho\right|_{G^{n}}$ are all equivalent to $\rho_{\nu}$. Then there is a representation $\pi$ of the semigroup $H\left(h_{0}\right)$ such that $\rho$ is equiralent to $\rho_{\nu} \sim \otimes \pi$.

This is a version of a standard result found, e.g., in [6], Theorem 5.1.7. Although $G^{n}$ may not be a finite group or $H\left(h_{0}\right)$ may not be a group, an argument analogous to the proof of [loc. cit.] works because of our assumptions, i.e. the finite dimensionality of $V$ and the complete reducibility of $\left.\rho\right|_{G^{n}}$.

1.4. The characters associated with the parallel version. Fix a $\boldsymbol{C}$-valued function $f$ on $B$ of trace type and $\boldsymbol{r}$ a positive integer. The $r$-parallel version $f^{(r)}$ of $f$ is also of trace type since we have the following from (1.1.5).

$$
f^{(r)}\left((b, n)^{\wedge}\right)=\sum_{\mu \in A_{r n}^{(f)}} a_{\mu}(f) \chi_{\mu}\left(\phi_{n}^{(r)}(b)\right) .
$$

We decompose the characters $\chi_{\mu} \circ \phi_{n}^{(r)}\left(\mu \in A_{r n}(f)^{\wedge}\right)$ into sums of characters of $B_{n}$ of smaller degrees. We need some preparations. Let $\iota_{j}: C B_{r} \rightarrow C B_{r n}(1 \leq j \leq n)$ be the homomorphism defined by

$$
\iota_{j}\left(\sigma_{i}\right)=\sigma_{i+r j-r} \quad(1 \leq i \leq r-1)
$$

and $\iota: C B_{r}^{\otimes n} \rightarrow \boldsymbol{C} B_{r n}$ the homomorphism defined by $\iota\left(b_{1} \otimes \cdots \otimes b_{n}\right)=\iota_{1}\left(b_{1}\right) \cdots \iota_{n}\left(b_{n}\right)$ for $b_{j} \in C B_{r}$, where $C B_{r}^{\otimes n}$ denotes the $n$-fold tensor product $C B_{r} \otimes \cdots \otimes C B_{r}$ of $\boldsymbol{C} B_{r}$. We regard $\boldsymbol{C} B_{r}^{\otimes n}$ as a subalgebra of $\boldsymbol{C} B_{r n}$ by the inclusion $\iota$. Let $\theta$ be the group homomorphism from $B_{n}$ to the symmetric group $S_{n}$ of degree $n$ defined by $\theta\left(\rho_{i}\right)=(i i+1)$ (the transposition of $i$ and $\left.i+1\right)$. We define an action of $B_{n}$ on $\Omega=\{1, \cdots, n\}$ by $b(i)=\theta(b)(i)$ for $i \in \Omega$. Then we have the following.

Lemma 1.4.3. For $b \in B_{n}$ and $b^{\prime} \in B_{r}$,

$$
\phi_{n}^{(r)}(b) \iota_{k}\left(b^{\prime}\right)=\iota_{b(k)}\left(b^{\prime}\right) \phi_{n}^{(r)}(b) \quad(1 \leq k \leq n) .
$$

Proof. The following formulas imply the statement of the lemma. 
(1.4.4. a) $\quad \phi_{n}^{(r)}\left(\sigma_{i}\right) \iota_{k}\left(\sigma_{j}\right)=\iota_{k}\left(\sigma_{j}\right) \phi_{n}^{(r)}\left(\sigma_{i}\right) \quad(1 \leq i \leq n-1,1 \leq j \leq r-1, k \neq i, i+1)$,

(1.4.4.b) $\phi_{n}^{(r)}\left(\sigma_{i}\right) \iota_{i+1}\left(\sigma_{j}\right)=\iota_{i}\left(\sigma_{j}\right) \phi_{n}^{(r)}\left(\sigma_{i}\right)$

$(1 \leq i \leq n-1,1 \leq j \leq r-1)$,

(1.4.4. c) $\phi_{n}^{(r)}\left(\sigma_{i}\right) \iota_{i}\left(\sigma_{j}\right)=\iota_{i+1}\left(\sigma_{j}\right) \phi_{n}^{(r)}\left(\sigma_{i}\right)$

$(1 \leq i \leq n-1,1 \leq j \leq r-1)$.

The formula (1.4.4a) is a consequence of (1.2.1) and the relations $\sigma_{i} \sigma_{j}=\sigma_{j} \sigma_{i}$ $(|i-j| \geq 2)$. We prove (1.4.4b). Let $\sigma^{\prime}(i, j)=\sigma_{i} \sigma_{i-1} \cdots \sigma_{j}(i \geq j)$. Then

$$
\begin{gathered}
\phi_{n}^{(r)}\left(\sigma_{i}\right)=\sigma(r i-r+1, r i-1)^{-r} \sigma^{\prime}(r i, r i-r+1) \sigma^{\prime}(r i+1, r i-r+2) \cdots \\
\sigma^{\prime}(r i+r-1, r i) .
\end{gathered}
$$

Since the relations of the braid group imply $\sigma^{\prime}(i, j) \sigma_{k}=\sigma_{k-1} \sigma^{\prime}(i, j)(i>k \geq j)$, we have

$$
\begin{aligned}
\phi_{n}^{(r)}\left(\sigma_{i}\right) \iota_{i+1}\left(\sigma_{j}\right) & \\
= & \sigma(r i-r+1, r i-1)^{-r} \sigma^{\prime}(r i, r i-r+1) \sigma^{\prime}(r i+1, r i-r+2) \cdots \\
& \sigma^{\prime}(r i+r-1, r i) \sigma_{r i+j} \\
= & \sigma(r i-r+1, r i-1)^{-r} \sigma_{r i-r+j} \sigma^{\prime}(r i, r i-r+1) \sigma^{\prime}(r i+1, r i-r+2) \cdots \\
& \sigma^{\prime}(r i+r-1, r i) .
\end{aligned}
$$

We also know ([1], p. 28, Corollary 1.8.4) that the element $\sigma(r i-r+1, r i-1)^{-r}$ is contained in the center of the subgroup $\left\langle\sigma_{r i-r+1}, \sigma_{r i-r+2}, \cdots, \sigma_{r i-1}\right\rangle$ of $B_{r n}$. Hence we have

$$
\phi_{n}^{(r)}\left(\sigma_{i}\right) \iota_{i+1}\left(\sigma_{j}\right)=\sigma_{r i-r+j} \phi_{n}^{(r)}\left(\sigma_{i}\right)=\iota_{i}\left(\sigma_{j}\right) \phi_{n}^{(r)}\left(\sigma_{i}\right) .
$$

This proves (1.4.4.b). The formula (1.4.4.c) is proved analogously by using $\phi_{n}^{(r)}\left(\sigma_{i}\right)=\sigma(r i-r+1, r i-1)^{-r} \sigma(r i, r i+r-1) \sigma(r i-1, r i+r-2) \cdots \sigma(r i-r+1, r i)$ and the relations $\sigma(i, j) \sigma_{k}=\sigma_{k+1} \sigma(i, j)(i \leq k<j)$.

Lemma 1.4.3 implies that the subgroup $\iota\left(B_{r}^{n}\right) \phi_{n}^{(r)}\left(B_{n}\right)$ of $B_{r n}$ is isomorphic to the wreath product $B_{r}^{n} \rtimes B_{n}$ with respect to $\theta_{n}: B \rightarrow S_{n}$.

Definition 1.4.5 (isotypic subspace). Let $A$ be a semisimple algebra over $\boldsymbol{C}, U$ an $A$-module and $\rho$ an irreducible representation of $A$. An $A$-submodule $W$ of $U$ is called the $\rho$-isotypic subspace if $W$ is the maximal subspace on which the action of $A$ is isomorphic to $\rho \oplus \cdots \oplus \rho$ ( $n$-times) for some $n=0,1,2, \cdots$.

For $\nu(1), \cdots, \nu(n) \in A_{r}(f)^{\wedge}$ and $\mu \in A_{r n}(f)^{\wedge}$, let $V_{\mu, \nu(1), \cdots, \nu(n)}$ be the $\rho_{\nu(1)} \otimes \cdots$ $\otimes \rho_{\nu(n)}$-isotypic subspace of $V_{\mu}$ as a $B_{r}^{n}$-module and $Y=V_{\nu(1)} \otimes \cdots \otimes V_{\nu(n)}$. Then $V_{\mu, \nu(1), \cdots, \nu(n)} \cong Y \oplus \cdots \oplus Y \cong Y \otimes C^{d(\mu, \nu(1), \cdots, \nu(n))}$, where $d(\mu, \nu(1), \cdots, \nu(n))$ denote

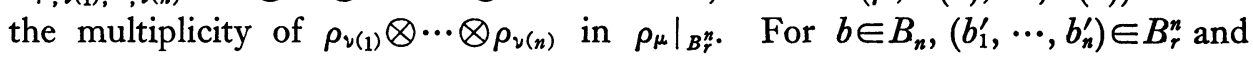
$v_{1} \otimes \cdots \otimes v_{n} \otimes w \in V_{\nu(1)} \otimes \cdots \otimes V_{\nu(n)} \otimes C^{d(\mu \nu(1), \cdots, \nu(n))}=V_{\mu, \nu(1), \cdots, \nu(n)}$, we have 


$$
\begin{aligned}
& \rho_{\mu}\left(\iota\left(b_{1}^{\prime} \otimes \cdots \otimes b_{n}^{\prime}\right) \phi_{n}^{(r)}(b)\right)\left(v_{1} \otimes \cdots \otimes v_{n} \otimes w\right) \\
& \quad=\rho_{\mu}\left(\phi_{n}^{(r)}(b) \iota\left(b_{b(1)}^{\prime} \otimes \cdots \otimes b_{b(n)}^{\prime}\right)\right)\left(v_{1} \otimes \cdots \otimes v_{n} \otimes w\right) \\
& \quad=\rho_{\mu}\left(\phi_{n}^{(r)}(b)\right)\left(\rho_{\nu(1)}\left(b_{b(1)}^{\prime}\right) v_{1} \otimes \cdots \otimes \rho_{\nu(n)}\left(b_{b(n)}^{\prime}\right) v_{\nu} \otimes w\right),
\end{aligned}
$$

from (1.4.3). The above formula implies the following:

Lemma 1.4.7. For $b \in B_{n}$, the subspace $\rho_{\mu}\left(\phi_{n}^{(r)}(b)\right) V_{\mu, \nu(1), \ldots, \nu(n)}$ is equal to the $\rho_{\nu\left(b^{-1}(1)\right)} \otimes \cdots \otimes \rho_{v\left(b^{-1}(n)\right)}$-isotypic subspace $V_{\mu, v\left(b^{-1}(1)\right), \cdots, \nu\left(b^{-1}(n)\right)}$ of $V_{\mu}$.

In particular, $V_{\mu, \nu, \cdots, \nu}$ is invariant relative to the actiono of $\iota\left(B_{r}^{n}\right) \phi_{n}^{(r)}\left(B_{n}\right)$. Let $\rho_{\mu, \nu}$ be the representation of $\iota\left(B_{r}^{n}\right) \phi_{n}^{(r)}\left(B_{n}\right)$ obtained by restricting $\rho_{\mu}$ on $V_{\mu, \nu, \cdots, \nu}$ and $\chi_{\mu, \nu}$ its character. Then we have the following.

Proposition 1.4.8. For $b^{\prime} \in B_{r}$ and $b \in B_{n}$ such that $b^{\wedge}$ is a knot, we have

$$
\chi_{\mu}\left(\iota_{1}\left(b^{\prime}\right) \phi_{n}^{(r)}(b)\right)=\sum_{\mu \in A_{r}(f) \wedge} \chi_{\mu, \nu}\left(\iota_{1}\left(b^{\prime}\right) \phi_{n}^{(r)}(b)\right) .
$$

Proof. Recall that $f$ is of trace type. The condition (ii) of Definition 1.1.7 implies that there are algb algebra homomorphisms $\iota_{i} \sim: A_{r}(f) \rightarrow A_{r n}(f)(1 \leq i \leq n)$ such that $\iota_{i} \sim \circ p_{r}=p_{r n} \circ \iota_{i}$. Hence the action of $B_{r}^{n}$ on $V_{\mu}$ is factored by $p_{r}^{n}: B_{r}^{n} \rightarrow$ $A_{r}(f)^{n}$. Since $A_{r}(f)$ is the associated algebra of $f, A_{r}(f)$ is completey reducible and so we have $V_{\mu}=\bigoplus_{\nu \in\left(A_{r}(f)^{n}\right)^{\wedge}} V_{\mu, \nu(1), \cdots, \nu(n)}$. Lemma 1.4.7 implies that

$$
\begin{aligned}
\rho_{\mu}\left(\iota_{1}\left(b^{\prime}\right) \phi_{n}^{(r)}(b)\right) V_{\mu, \nu(1), \cdots, \nu(n)} & =\rho_{\mu}\left(\iota_{1}\left(b^{\prime}\right)\right) V_{\mu, \nu\left(b^{-1}(1)\right), \cdots, \nu\left(b^{-1}(n)\right)} \\
& =V_{\mu, \nu\left(b^{-1}(1)\right), \cdots, \mu\left(b^{-1}(n)\right)} .
\end{aligned}
$$

Hence if $V_{\mu, \nu(1), \cdots, \nu(n)} \in V_{\mu, \nu\left(b^{-1}(()), \cdots, \nu\left(b^{-1}(n)\right)\right.}$, then the diagonal part of the matrix $\iota_{1}\left(b^{\prime}\right) \phi_{n}^{(r)}(b)$ corresponding to the subspace $V_{\mu, \nu(1), \cdots, \nu(n)}$ is equal to 0 . Thus $\chi_{\mu}\left(c_{1}\left(b^{\prime}\right) \phi_{n}^{(r)}(b)\right)$ is equal to the sum of the diagonal elements of $\rho_{\mu}\left(\iota_{1}\left(b^{\prime}\right) \phi_{n}^{(r)}(b)\right)$ corresponding to the subspaces $V_{\mu, \nu(1), \cdots, \nu(n)}$ with $V_{\mu, \nu(n), \mu, \nu(n)}=V_{\mu, \nu\left(b^{-1}(1)\right), \cdots, \nu\left(b^{-1}(n)\right)}$. Since the closure of $b$ is a knot, $\theta(b)$ is an $n$-cycle. Thus $\nu(i)=\nu\left(b^{-1}(i)\right)(1 \leq i \leq n)$ imply $\nu(1)=\cdots=\nu(n)$. But the sum of diagonals of $\rho_{\mu}\left(\iota_{1}\left(b^{\prime}\right) \phi_{n}^{(r)}(b)\right)$ on $V_{\mu, \nu, \cdots, \nu}$ is equal to $\chi_{\mu, \nu}\left(\iota_{1}\left(b^{\prime}\right) \phi_{n}^{(r)}(b)\right)$. This proves the proposition.

Since $\iota\left(B_{r}^{n}\right) \phi_{n}^{(r)}\left(B_{n}\right)$ is isomorphic to $B_{r}^{n} \backslash \backslash B_{n}$, we see, from Proposition 1.3.4, that ther is a representation $\pi_{\mu, \nu}$ of $B_{n}$ such that

$$
\rho_{\mu, \nu} \cong\left(\rho_{\nu}^{\otimes n}\right)^{\sim} \otimes \pi_{\mu, \nu}
$$

where $\left(\rho_{\nu}^{\otimes n}\right)^{\sim}$ is the representation of $\iota\left(B_{r}^{n}\right) \phi_{n}^{(r)}\left(B_{n}\right)$ coming from the representation $\rho_{\nu}^{\otimes n}$ of $B_{r}^{n}$ as in Proposition 1.3.4. Let $\chi_{\nu^{\sim}}, \omega_{\mu, \nu}$ be the characters of $\left(\rho_{\nu}^{\otimes n}\right)^{\sim}$, $\pi_{\mu, \nu}$ respectively. Now we can state our main theorem.

Theorem 1.4.10. Let $b$ be an n-braid whose closure is a knot. Then we have the following. 
(i) $\chi_{\mu}\left(\phi_{n}^{(r)}(b)\right)=\sum_{\nu \in A_{r}(f) \wedge} \chi_{\nu}(1) \omega_{\mu, \nu}(b)$,

(ii) $f^{(r)}(b, n)=\sum_{\mu \in A_{r n}(f) \wedge}\left(\sum_{\nu \in A_{r}(f) \wedge} a_{\mu}(f) \chi_{\nu}(1) \omega_{\mu, \nu}(b)\right)$.

Proof. Proposition 1.4.8 and (1.4.9) yield that

$$
\chi_{\mu}\left(\phi_{n}^{(r)}(b)\right)=\sum_{\nu \in A_{r}(f) \wedge} \chi_{\mu, \nu}\left(\phi_{n}^{(r)}(b)\right)=\sum_{\nu \in A_{r}(f) \wedge} \chi_{\nu}^{\sim}\left(\phi_{n}^{(r)}(b)\right) \omega_{\mu, \nu}(b) .
$$

Hence the first formula is an immediate consequence of the following lemma with $b^{\prime}=1$. The second one is obtained from (i) and (1.4.1).

Lemma 1.4.11. For $b^{\prime} \in B_{r}$ and $b \in B_{n}$ such that $b^{\wedge}$ is a knot, we have

$$
\chi_{\nu} \sim\left(\iota_{1}\left(b^{\prime}\right) \phi_{n}^{(r)}(b)\right)=\chi_{\nu}\left(b^{\prime}\right) .
$$

Proof. By using 1.3.3, we have

$$
\begin{aligned}
& \chi_{\nu} \sim\left(c_{1}\left(b^{\prime}\right) \phi_{n}^{(r)}(b)\right) \\
& \quad=\sum_{\alpha_{1}, \beta_{1}, \cdots, \alpha_{n}, \beta_{n}} \delta_{\alpha_{1} \beta_{1}} \cdots \delta_{\alpha_{n} \beta_{n}}\left(\rho_{\nu}\left(b^{\prime}\right)_{\alpha_{\alpha_{1} \beta_{b}-1(1)}} \rho_{\nu}(1)_{\alpha_{2} \beta_{b}-1(2)} \cdots \rho_{\nu}(1)_{\alpha_{n} \beta_{b}-1(n)}\right) \\
& \quad=\sum_{\alpha_{1}, \beta_{1}, \cdots, \alpha_{n}, \alpha_{n}} \delta_{\alpha_{1} \beta_{1}} \cdots \delta_{\alpha_{n} \beta_{n}}\left(\rho_{\nu}\left(b^{\prime}\right)_{\alpha_{1_{1} \beta_{b}-1(1)}} \delta_{\alpha_{\alpha_{2} \beta_{b}-1(2)}} \cdots \delta_{\alpha_{n} \beta_{b}-1(n)}\right) .
\end{aligned}
$$

Since $b^{\wedge}$ is a knot and so $\theta(b)$ is an $n$-cycle, each term of the above summention is 0 except when $\alpha_{1}=\beta_{b^{-1}(1)}=\alpha_{b^{-1}(1)}=\cdots=\alpha_{b^{-n+1}(1)}=\beta_{1}$, i.e. $\alpha_{1}, \cdots, \alpha_{n}$, $\beta_{1}, \cdots, \beta_{n}$ are all equal. Hence we have

$$
\chi_{\nu} \sim\left(c_{1}\left(b^{\prime}\right) \phi_{n}^{(r)}(b)\right)=\sum_{\alpha_{1}} \rho_{\nu}\left(b^{\prime}\right)_{\alpha_{1} \alpha_{1}}=\chi_{\nu}\left(b^{\prime}\right) .
$$

This completes the proof.

1.5. A decomposition of $\boldsymbol{X}^{(r)}$ into invariants. For a $\boldsymbol{C}$-valued function $f$ on $B$ of trace type, let

$$
f^{(r, v)}(b, n)=\sum_{\mu \in A_{r n}(f) \wedge} a_{\mu}(f) \omega_{\mu, \nu}(b) .
$$

The $r$-parallel version $X^{(r)}$ of a link invariant $X$ of trace type is a sum of invariants $X^{(r, \nu)}$ parametrized by $A_{r}(X)^{\wedge}$ :

Theorem 1.5.1. For a $C$-valued function $f$ on $B$ of trace type and $b \in B_{n}$, we have the following

(i) $f^{(r, v)}$ is a link invariant if $f$ is a link invariant.

(ii) $f^{(r)}(K)=\sum_{\nu \in A_{r}(f)} \chi_{\nu}(1) f^{(r, \nu)}(K)$ for a knot $K$.

Proof. (ii) is an immediate consequence of the definition of $f^{(r, v)}$ and Theorem 1.4.10 (ii). To show (i), we check the invariance of $f^{(r, v)}$ relative to the relations (i), (ii) of Definition 1.1.2. Let $b$ and $b^{\prime}$ be elements of $B_{n}$. Since 
$\omega_{\mu, \nu}\left(b b^{\prime}\right)=\omega_{\mu, \nu}\left(b^{\prime} b\right)$, we have $f^{(r, \nu)}\left(b b^{\prime}, n\right)=f^{(r, \nu)}\left(b^{\prime} b, n\right)$. It remains to show that

$$
f^{(r, \nu)}(b, n)=f^{(r, \nu)}\left(b \sigma_{n}^{ \pm 1}, n+1\right) .
$$

Let $f_{n}: \boldsymbol{C} B_{n} \rightarrow \boldsymbol{C}$ be a $\boldsymbol{C}$-linear function defined by $f_{n}(b)=f(b, n)$ for $b \in B_{n}$. For $\nu \in A(f)^{\wedge}$, let $\eta_{\nu}$ be an element of $\boldsymbol{C} B_{r}$ such that $\rho_{\nu}\left(\eta_{\nu}\right)=$ id and $\rho_{\nu}\left(\eta_{\nu}\right)=0$ for $\nu \neq \nu^{\prime} \in A_{r}(f)^{\wedge}$. Then $p_{r}\left(\eta_{v}\right)$ is contained in the center of $A_{r}(f), p_{r}\left(\eta_{v}\right)^{2}=p_{r}\left(\eta_{v}\right)$, and $p_{r}\left(\eta_{\nu}\right) p_{r}\left(\eta_{\nu^{\prime}}\right)=0$ if $\nu \neq \nu^{\prime}$. Since $\rho_{\mu}\left(\iota_{1}\left(\eta_{\nu}\right) \iota_{2}\left(\eta_{\nu}\right) \cdots \iota_{n}\left(\eta_{\nu}\right)\right)$ is a projection from $V_{\mu}$ to the $\rho_{\nu}^{\otimes n}$-isotypic subspace $V_{\mu, \nu, \cdots, \nu}$ of $V_{\mu}$, we have $\rho_{\mu}\left(\iota_{1}\left(\eta_{\nu}\right) \iota_{2}\left(\eta_{\nu}\right) \cdots \iota_{n}\left(\eta_{\nu}\right) \phi_{n}^{(r)}(b)\right)$ $=\rho_{\mu, \nu}\left(\phi_{n}^{(r)}(b)\right)$. Hence by (1.4.9) and Lemma 1.4.11, we get

$$
\begin{aligned}
& \omega_{\mu, \nu}(b)=\chi_{\nu}(1)^{-1} \chi_{\mu}\left(\iota_{1}\left(\eta_{\nu}\right) \iota_{2}\left(\eta_{\nu}\right) \cdots \iota_{n}\left(\eta_{\nu}\right) \phi_{n}^{(r)}(b)\right) \\
& \text { for } \mu \in A_{r n}(f)^{\wedge} \text { and } \nu \in A_{r}(f)^{\wedge} .
\end{aligned}
$$

This formula implies that

$$
\begin{aligned}
& f^{(r, \nu)}\left(b \sigma_{n}^{ \pm 1}, n+1\right) \\
& \quad=\chi_{\nu}(1)^{-1} f_{r n+r}\left(\iota_{1}\left(\eta_{v}\right) \cdots \iota_{n}\left(\eta_{v}\right) \iota_{n+1}\left(\eta_{v}\right) \phi_{n+1}^{(r)}\left(b \sigma_{n}^{ \pm 1}\right)\right) .
\end{aligned}
$$

But we know, from Lemma 1.4.3, that $\iota_{n+1}\left(\eta_{\nu}\right) \phi_{n}^{(r)}\left(b \sigma_{n}^{ \pm 1}\right)=\phi_{u+1}^{(r)}\left(b \sigma_{r}^{ \pm 1}\right) \iota_{n}\left(\eta_{\nu}\right)$ and so we have

$$
\begin{aligned}
f^{(r, \nu)} & \left(b \sigma_{n}^{ \pm 1}, n+1\right) \\
& =\chi_{\nu}(1)^{-1} f_{r n+r}\left(\iota_{1}\left(\eta_{\nu}\right) \cdots \iota_{n}\left(\eta_{\nu}\right) \phi_{n+1}^{(r)}\left(b \sigma_{n}^{ \pm 1}\right) \iota_{n}\left(\eta_{\nu}\right)\right) \\
& =\chi_{\nu}(1)^{-1} f_{r n+r}\left(\iota_{n}\left(\eta_{\nu}\right) \iota_{1}\left(\eta_{\nu}\right) \cdots \iota_{n}\left(\eta_{\nu}\right) \phi_{n+1}^{(r)}\left(b \sigma_{n}^{ \pm 1}\right)\right) \\
& =\chi_{\nu}(1)^{-1} f_{r n+r}\left(\iota_{1}\left(\eta_{\nu}\right) \cdots \iota_{n-1}\left(\eta_{\nu}\right) \iota_{n}\left(\eta_{\nu}\right) \phi_{n+1}^{(r)}\left(b \sigma_{n}^{ \pm 1}\right)\right) . \\
& =\chi_{\nu}(1)^{-1} f_{r n+r}\left(\iota_{1}\left(\eta_{\nu}\right) \cdots \iota_{n-1}\left(\eta_{\nu}\right) \iota_{n}\left(\eta_{\nu}\right) \phi_{n}^{(r)}\left(b \sigma_{n}^{ \pm 1}\right)\right) .
\end{aligned}
$$

In the last step of the above calculation, we use $p_{r}\left(\eta_{\nu}\right)^{2}=p_{r}\left(\eta_{\nu}\right)$. Since $\phi_{n}^{(r)}\left(\sigma_{n}^{ \pm 1}\right)=$ $\left(\sigma_{n r}^{(r)}\right)^{ \pm 1}$, Lemma 1.2.4 implies that the last term of the above is equal to $\chi_{\nu}(1)^{-1} f_{r n+r}\left(\iota_{1}\left(\eta_{v}\right) \cdots \iota_{n-1}\left(\eta_{v}\right) \iota_{n}\left(\eta_{v}\right) \phi_{n}^{(r)}(b)\right)$, which is equal to $f^{(r, \nu)}(b, n)$ by (1.5.3). Hence we have (1.5.2).

1.6. Invariants of cable links. Let $\iota_{i}: B_{r} \rightarrow B_{r n}(1 \leq i \leq n)$ be the group homomorphism defined in Section 1.4.

Proposition 1.6.1. Let $b^{\prime} \in B_{r}$ and $\sim$ denote the Markov equivalence relation (Definition 1.1.2). Then we have the following:

(a) For $b_{1}, b_{2} \in B_{n}$ such that the closure of $b_{1} b_{2}$ is a knot, $\left(\iota_{1}\left(b^{\prime}\right) \phi_{n}^{(r)}\left(b_{1} b_{2}\right), r n\right) \sim$ $\left(c_{1}\left(b^{\prime}\right) \phi_{n}^{(r)}\left(b_{2} b_{1}\right), r n\right)$,

(b) For $b \in B_{n},\left(\iota_{1}\left(b^{\prime}\right) \phi_{n}^{(r)}(b), r n\right) \sim\left(\iota_{1}\left(b^{\prime}\right) \phi_{n+1}^{(r)}\left(b \sigma_{n}^{ \pm 1}\right), r n+r\right)$.

Proof. Lemma 1.2.4 shows the part (b). By using Definition 1.1.2 (ii) and Lemma 1.4.3, we have 


$$
\begin{aligned}
& \left(\iota_{1}\left(b^{\prime}\right) \phi_{n}^{(r)}\left(b_{1} b_{2}\right), r n\right) \sim\left(\phi_{n}^{(r)}\left(b_{1} b_{2}\right)\left(b^{\prime}\right), r n\right) \\
& \quad=\left(\iota_{b_{1} b_{2}(1)}\left(b^{\prime}\right) \phi_{n}^{(r)}\left(b_{1} b_{2}\right), r n\right) \sim \cdots \\
& \quad \sim\left(\iota_{\left(b_{1} b_{2}\right)^{k}(1)}\left(b^{\prime}\right) \phi_{n}^{(r)}\left(b_{1} b_{2}\right), r n\right) \quad(k=1,2, \cdots) \\
& \quad \sim\left(\iota_{b_{2}\left(b_{1} b_{2}\right)^{k}(1)}\left(b^{\prime}\right) \phi_{n}^{(r)}\left(b_{2} b_{1}\right), r n\right) .
\end{aligned}
$$

Since the closure of $b_{1} b_{2}$ is a knot, we have $\left\{\left(b_{1} b_{2}\right)^{k}(1) \mid k=0,1, \cdots, n-1\right\}=$ $\{1, \cdots, n\}$ and so there is $k^{\prime}$ with $\left(b_{1} b_{2}\right)^{k^{\prime}}(1)=b_{2}^{-1}(1)$. Hence we have $\left(\iota_{b_{2}\left(b_{1} b_{2}\right)^{k^{\prime}(1)}}\left(b^{\prime}\right) \phi_{n}^{(r)}\left(b_{2} b_{1}\right), r n\right)=\left(\iota_{1}\left(b^{\prime}\right) \phi_{n}^{(r)}\left(b_{2} b_{1}\right), r n\right)$. This proves the part (a).

The above proposition and Theorem 1.1.3 yield the following.

Corollary 1.6.2. Let $b^{\prime} \in B_{r}$ and $\left(b_{1}, n_{1}\right),\left(b_{2}, n_{2}\right)$ be two braids such that the closures of $b_{1}$ and $b_{2}$ are knots. Then the closures of $\left(\iota_{1}\left(b^{\prime}\right) \phi_{n_{1}}^{(r)}\left(b_{1}\right), r n_{1}\right)$ and $\left(c_{1}\left(b^{\prime}\right) \phi_{n_{2}}^{(r)}\left(b_{2}\right), r n_{2}\right)$ are equivalent if $\left(b_{1}, n_{1}\right)^{\wedge}$ and $\left(b_{2}, n_{2}\right)^{\wedge}$ are equivalent.

Definition 1.6.3 (cable links). For a braid $(b, n)$ and $b^{\prime} \in B_{r}$, we call $\left(c_{1}\left(b^{\prime}\right) \phi_{n}^{(r)}(b), r n\right)^{\wedge}$ the $(r$-strand $)$ cable link of $(b, n)^{\wedge}$ associated with $b^{\prime}$. For a function $f$ on $B$, let $f_{b^{\prime}}^{(r)}(b, n)=f\left(\iota_{1}\left(b^{\prime}\right) \phi_{n}^{(r)}(b), r n\right)$.

The $r$-strand cable links is well-defined by Corollary 1.6.2, and so $X_{b^{\prime}}^{(r)}$ is an invariant of link isotopy types for a link invariant $X$ and $b^{\prime} \in C B_{r}$.

Theorem 1.6.4. Let $b^{\prime} \in C B_{r}$. For a braid $b \in B_{n}$ whose closure is a knot and $a \boldsymbol{C}$-valued function $f$ on $B$ of trace type, $f_{b^{\prime}}^{(r)}$ satisfies

$$
f_{b^{\prime}}^{(r)}(b, n)=\sum_{\nu \in A_{r}(f)^{\wedge}} \chi_{\nu}\left(b^{\prime}\right) f^{(r, \nu)}(b, n)
$$

Proof. Since $f_{b^{\prime}}^{(r)}(b, n)=\sum_{\mu \in A_{r n}(f) \wedge} \chi_{\mu}\left(\iota_{1}\left(b^{\prime}\right) \phi_{n}^{(r)}(b)\right)$, we get the stateent of the theorem by (1.4.9) and Lemma 1.4.11.

\section{The parallel version of link invariants, II (the case of general} links). In this section, we give a generalization of Theorems 1.4.10 and 1.6.4 for braids whose closures are links.

2.1. The characters associated with the parallel version. Fix a $C$-valued function $f$ on $B$ of trace type and fix an element $b_{0} \in B_{n}$. Let $B_{n}\left(b_{0}\right)=\left\{b \in B_{n} \mid\right.$ every $\langle\theta(b)\rangle$-orbit of $\Omega$ is contained in a single $\left\langle\theta\left(b_{0}\right)\right\rangle$-orbit of $\Omega\}$, where $\Omega=\{1, \cdots, n\}$. We use the notations in Sections 1.3 and 1.4. Lemma 1.4.3 implies that the subgroup $\iota\left(B_{r}^{n}\right) \phi_{n}^{(r)}\left(B_{n}\left(b_{0}\right)\right)$ of $B_{r n}$ is isomorphic to the wreath product $B_{r}^{n} \backslash B_{n}\left(b_{0}\right)$ with respect to $\theta: B_{n} \rightarrow S_{n}$. For $\nu=\left(\nu_{1}, \cdots, \nu_{n}\right)$ $\left(\nu_{i} \in A_{r}(f)^{\wedge}\right)$, let $\rho_{\nu}=\rho_{\nu_{1}} \otimes \cdots \otimes \rho_{\nu_{n}}$ be the representation of $B_{r}^{n}$. For $\mu \in A_{r n}(f)^{\wedge}$, let $V_{\mu, \nu}$ be the $\rho_{\nu}$-isotypic subspace of $V_{\mu}$ as a $C B_{r n}$-module Let $\delta(j)=$ $\min \left\langle\theta\left(b_{0}\right)\right\rangle \cdot j$ for $j \in \Omega$. If $\nu_{j}=\nu_{\delta(j)}$ for $1 \leq j \leq n$, then by Lemma $1.4 .3, V_{\mu, \nu}$ is 
invariant relative to the action of $B_{r}^{n} \backslash B_{n}\left(b_{0}\right)$. In this case, let $\rho_{\mu, \nu}$ denote the representation of $\iota\left(B_{r n}\right) \phi_{n}^{(r)}\left(B_{n}\left(b_{0}\right)\right)$ obtained by restricting $\rho_{\mu}$ on $V_{\mu, \nu}$ and $\chi_{\mu, \nu}$ its character. Then we have the following.

Proposition 2.1.1. Let $k$ be the number of $\left\langle\theta\left(b_{0}\right)\right\rangle$-orbits of $\Omega$ and $\{\delta(j) \mid j \in \Omega\}=\left\{m_{1}, \cdots, m_{k}\right\}$. Then we have

$$
\chi_{\mu}\left(\phi_{n}^{(r)}\left(b_{0}\right)\right)=\sum_{v_{m_{1}} \in A_{r}(f)^{\wedge}} \sum_{v_{m_{k}} \in A_{r}(f)^{\wedge}} \chi_{\mu,\left(v_{\delta(1)} \cdots, \nu_{\delta(n)}\right.} \phi_{n}^{(r)}\left(\left(b_{0}\right)\right) .
$$

Proof. The proof of Proposition 1.4.8 shows that $\chi_{\mu}\left(\phi_{n}^{(r)}\left(b_{0}\right)\right)$ is equal to the sum of the diagonal elements of $\rho_{\mu}\left(\phi_{n}^{(r)}\left(b_{0}\right)\right)$ corresponding to the subspaces

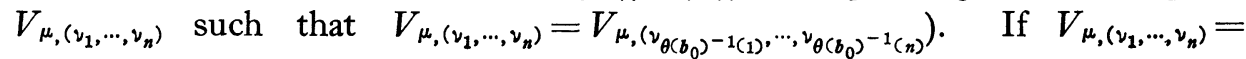
$V_{\mu,\left(\nu_{\theta\left(b_{0}\right)^{-1}(1)}, \cdots, \nu_{\theta\left(b_{0}\right)^{-1}(n)}\right)}$, then we have $\nu_{j}=\nu_{\delta(j)}$ and the sum of diagonal elements of $\rho_{\mu}\left(\phi_{n}^{(r)}\left(b_{0}\right)\right)$ corresponding to the subspace $V_{\mu,\left(v_{1}, \cdots, v_{n}\right)}$ is equal to $\chi_{\mu,\left(v_{\delta(1)}, \cdots, v_{\delta(n)}\right)} \phi_{n}^{(r)}\left(\left(b_{0}\right)\right)$. Hence we get conclusion.

Let $\nu=\left(\nu_{1}, \cdots, \nu_{n}\right) \in\left(A_{r}(f)^{\wedge}\right)^{n}$ with $\nu_{j}=\nu_{\delta(j)}$. Since $\iota\left(B_{r}^{n}\right) \phi_{n}^{(r)}\left(B_{n}\left(b_{0}\right)\right)$ is isomorphic to $B_{r}^{n} \rtimes B_{n}\left(b_{0}\right)$, we see, from Proposition 1.3.4, that there is a representation $\pi_{\mu, \nu}$ of $B_{n}$ such that

$$
\rho_{\mu, \nu} \cong \rho_{\nu} \sim \otimes \pi_{\mu, \nu},
$$

where $\rho_{\nu} \sim$ is the representation of $\iota\left(B_{r}^{n}\right) \phi_{n}^{(r)}\left(B_{n}\left(b_{0}\right)\right)$ coming from the representation $\rho_{\nu}$ of $B_{r}^{n}$ as in Proposition 1.3.4. Let $\chi_{\nu} \sim, \omega_{\mu, \nu}$ be the characters of $\rho_{\nu}$, $\pi_{\mu, \nu}$ respectively. Now we can state a generalized version of Theorem 1.4.10.

Theorem 2.1.3. (i) For $\mu \in A_{r n}(f)^{\wedge}$,

$$
\chi_{\mu}\left(\phi_{n}^{(r)}\left(b_{0}\right)\right)=\sum_{\nu_{m_{1}} \in A_{r}(f)^{\wedge}} \sum_{v_{m_{k}} \in A_{r}(f)^{\wedge}}\left(\prod_{j=1}^{k} \chi_{\nu_{m_{j}}}(1)\right) \omega_{\mu,\left(\nu_{\delta(1)}, \cdots, \nu_{\delta(n)}\right.}\left(b_{0}\right)
$$

(ii)

$$
f^{(r)}\left(b_{0}\right)=\sum_{\mu \in A_{r n}(f)^{\wedge}} \sum_{\nu_{m_{1}} \in \Delta_{r}(f) \wedge} \ldots \sum_{\nu_{m_{k}} \in A_{r}(f)^{\wedge}} a_{\mu}(f)\left(\prod_{j=1}^{k} \chi_{\nu_{m_{j}}}(1)\right) \omega_{\mu,\left(v_{\delta(1)}, \cdots, \nu_{\delta(n)}\right.}\left(b_{0}\right) .
$$

Proof. Proposition 2.1.1 and (2.1.2) yield that

$$
\begin{aligned}
& \chi_{\mu}\left(\phi_{n}^{(r)}\left(b_{0}\right)\right)=\sum_{\nu_{m_{1}} \in A_{r}(f) \wedge} \cdots \sum_{\nu_{m_{k}} \in A_{r}(f) \wedge} \chi_{\mu_{,}\left(v_{\delta(1)}, \cdots, v_{\delta(n)}\right)}\left(b_{0}\right) \\
& =\sum_{\nu_{m_{1}} \in A_{r}(t) \wedge} \ldots \sum_{\nu_{m_{k}} \in A_{r}(f) \wedge}\left(\chi_{\nu} \sim\left(\phi_{n}^{(r)}\left(b_{0}\right)\right)\right) \omega_{\mu,\left(\nu_{\delta(1)}, \cdots, \nu_{\delta(n)}\right.}\left(b_{0}\right) \text {. }
\end{aligned}
$$

Hence the first formula is an immediate consequence of following Lemma 2.1.4. The second one is obtained from (i) and (1.4.1).

Lemma 2.1.4. We have 


$$
\chi_{\nu} \sim\left(b_{0}\right)=\prod_{j=1}^{k} \chi_{v_{m_{j}}}(1)
$$

Proof. By using (1.3.3), we have

$$
\begin{aligned}
& \chi_{\nu} \sim \phi_{n}^{(r)}\left(\left(b_{0}\right)\right) \\
& \quad=\sum_{\alpha_{1}, \beta_{1} \cdots, \alpha_{n}, \beta_{n}} \delta_{\alpha_{1} \beta_{1}} \cdots \delta_{\alpha_{n} \beta_{n}}\left(\rho_{\nu_{1}}(1)_{\alpha_{\theta\left(b_{0}\right)-1(1)}} \rho_{\nu_{2}}(1)_{\alpha_{2} \beta_{\theta\left(b_{0}\right)-1(2)}} \cdots \rho_{\nu_{n}}(1)_{\alpha_{n} \beta_{\theta\left(b_{0}\right)-1(n)}}\right) .
\end{aligned}
$$

Let $|S|$ denote the cardinarity of a set $S$. Each term of the above summention is zero unless

$$
\alpha_{m_{1}}=\beta_{\theta\left(b_{0}\right)^{-1}\left(m_{j}\right)}=\alpha_{\theta\left(b_{0}\right)^{-1}\left(m_{j}\right)}=\cdots=\alpha_{\theta\left(b_{0}\right)^{-\left|\psi^{-1}(j)\right|+1\left(m_{j}\right)}}=\beta_{m_{j}} \quad(1 \leq j \leq k) .
$$

But the above equalities implies that $\alpha_{i}=\beta_{i}=\alpha_{j}=\beta_{j}$ for $i$ and $j$ such that $\left\langle\theta\left(b_{0}\right)\right\rangle \cdot i=\left\langle\theta(b /)_{0}\right\rangle \cdot j$. Hence we have

$$
\chi_{\nu} \sim\left(b_{0}\right)=\prod_{j=1}^{k}\left(\sum_{\alpha_{m_{j}}} \rho_{\nu_{j}}(1)_{\alpha_{m_{j}}, \alpha_{m_{j}}}\right)=\prod_{j=1}^{k} \chi_{\nu_{j}}(1)
$$

This proves Lemma 2.1.4.

2.2. Further generalizations. In this section, we state a generalized version of Theorem 1.6.4 for cable links of multi-component links. We omit proofs of the results in this section, which are anologous to those of corresponding facts in Section 1.

Let $K$ be a $k$-component link. A bijection $\Psi$ from the set of connected components of $K$ to $\{1,2, \cdots, k\}$ is called a marking of $K$ and the pair $(K, \Psi)$ is called a (k-component) marked link. The connected component $C$ of $K$ with $\Psi(C)=i$ is called the $i$-th component of $K$. Let $R=\left(r_{1}, r_{2}, \cdots, r_{k}\right) \in N^{k}$. Let $(K, \Psi)^{(R)}$ denote the link diagram obtained by replacing each crossing point of a link diagram of $K$ as in Figure 6. The link type of $(K, \Psi)^{(R)}$ is not depend on the choice of link diagrams of $K$. We call $(K, \Psi)^{(R)}$ the $R$-parallel version of $(K, \Psi)$. For a link invariant $X$, let $X^{(R)}(K, \Psi)=X\left((K, \Psi)^{(R)}\right)$. Then $X^{(R)}$ is an invariant of $k$-component marked links and is called the $R$-parallel version of $X$. We can show a generalized version of Theorem 2.1.3 (ii) for $X^{(R)}$. We omit the details.

Let $(K, \Psi)$ be a $k$-component marked link, $R=\left(r_{1}, r_{2}, \cdots, r_{k}\right) \in N^{k}$ and $b=$ $\left(b_{1}, b_{2}, \cdots, b_{k}\right) \in B_{r_{1}} \times \cdots \times B_{r_{k}}$. Let $(K, \Psi)_{b}$ denote the link obtained by inserting the braid $b_{i}(1 \leq i \leq k)$ to the bunch of the components of $(K, \Psi)^{(R)}$ corresponding to the component of $K$ sent to $i$ by $\Psi$ as in Figure 7 . We call $(K, \Psi)_{b}$ the cable link of $(K, \Psi)$ associated with $b$. For a link invariant $X$, let $X_{b}(K, \Psi)=$ $X\left((K, \Psi)_{b}\right)$. Then $X_{b}$ is an invariant of $k$-component marked links. Then we have:

Theorem 2.2.1. Let $X$ be a link invariant of trace type, $R=\left(r_{1}, r_{2}, \cdots, r_{k}\right) \in$ 

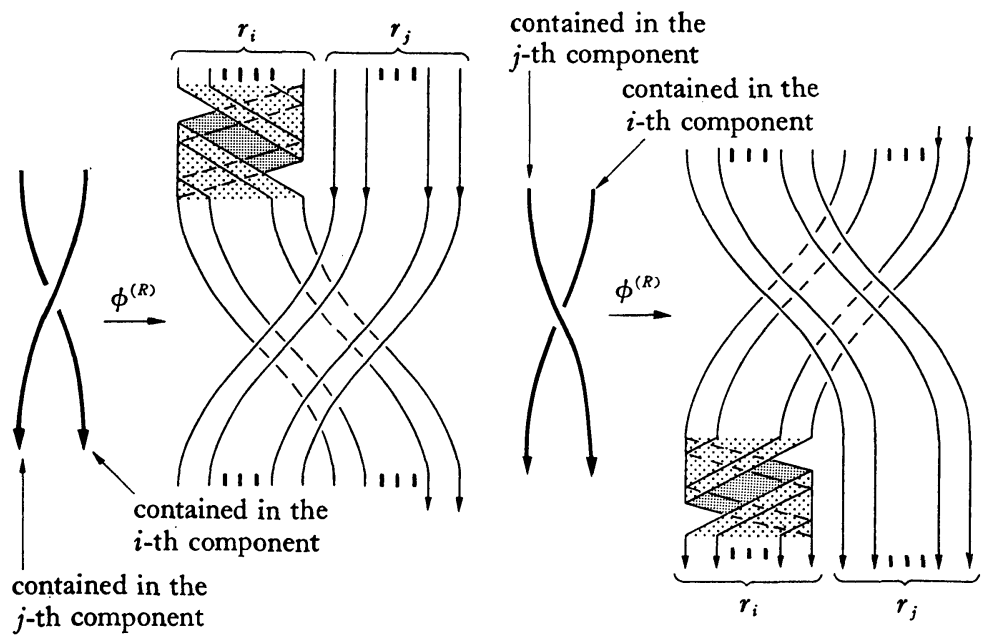

Figure 6

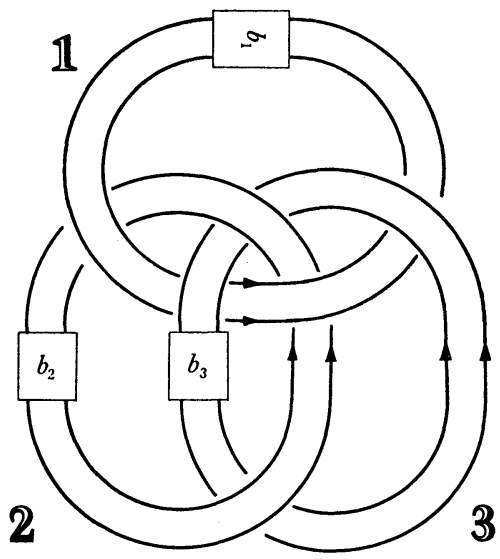

Figure 7

$N^{k}$ and $b=\left(b_{1}, b_{2}, \cdots, b_{k}\right) \in B_{r_{1}} \times \cdots \times B_{r^{k}}$. Then there are invariants $X^{(R, v)}$ of $k$ component marked links parametrized by $\nu=\left(\nu_{1}, \nu_{2}, \cdots, \nu_{k}\right) \in A_{r_{1}}(X)^{\wedge} \times \cdots \times A_{r_{k}}(X)^{\wedge}$ which satisfy the following. For a k-component marked link $(K, \Psi)$,

$$
X_{b}(K, \Psi)=\sum_{\left(\nu_{1}, \cdots, \nu_{k}\right) \in A_{r_{1}}(X)^{\wedge} \times \cdots \times A_{r_{k}}(X)^{\wedge}}\left(\prod_{j=1}^{k} \chi_{\nu_{i}}\left(b_{i}\right)\right) X^{(R, \nu)}(K, \Psi) .
$$

This theorem is a generalization of Theorems 1.5.1 and 1.6.4.

3. The r-parallel version of the two-variable Jones polynomial. In this section we review representation theory of Iwahori algebras and the two-varaible Jones polynomial. At the end of this section, we shall construct 
representations of $B_{3}$ assoiated with the two-parallel version of the two-variable Jones polynomial. Fix non-zero complex numbers $\alpha$ and $s$ such that $s$ is not equal to any root of unity. Let $q^{k / 2}=s^{k}$ for an integer $k$.

Definition 3.1 ([3], p. 55, ex. 23; [18]). Let $H_{n}(q)$ be a $\boldsymbol{C}$-algebra with a unit defined by the following relations:

$$
\begin{aligned}
& H_{n}(q)=\left\langle T_{1}, T_{2}, \cdots, T_{n-1}\right| T_{i}^{2}+(1-q) T_{i}-q=0 \quad(1 \leq i \leq n-1), \\
& T_{i} T_{i+1} T_{i}=T_{i+1} T_{i} T_{i+1} \quad(1 \leq i \leq n-2), \\
& T_{i} T_{j}=T_{j} T_{i} \quad(1 \leq i<j-1 \leq n-2)>.
\end{aligned}
$$

We call $H_{n}(q)$ the Iwahori's Hecke algebra of type $A_{n-1}$. For simplicity, we denote $H_{n}(q)$ by $H_{n}$ if there is no fear of confusion.

It is known that $H_{n}$ is semisimple (see, e.g. [11]) and isomorphic to the group algebra $\boldsymbol{C} S_{n}$ of the symmetric group $S_{n}$ of degree $n$.

Definition 3.3. Let $\Lambda(n)$ be the set of partitions of $n$, i.e.

$$
\Lambda(n)=\left\{\left(\lambda_{1}, \lambda_{2}, \cdots\right) \mid \sum_{i} \lambda_{i}=n, \lambda_{i} \in N \cup\{0\}, \lambda_{1} \geq \lambda_{i+1}(i \in N)\right\} .
$$

For $\lambda=\left(\lambda_{1}, \lambda_{2}, \cdots\right) \in \Lambda(n)$, let $\lambda_{i}^{*}=\max \left\{j \mid \lambda_{j} \geq i\right\}$ if $i \leq \lambda_{1}$ and $\lambda_{i}^{*}=0$ if $i>\lambda_{1}$. We call $\lambda^{*}=\left(\lambda_{1}^{*}, \lambda_{2}^{*}, \cdots\right)$ the dual partition of $\lambda$.

As is well-known, the irreducible representations of $S_{n}$ are a parametrized by $\Lambda(n)$. Hence $\Lambda(n)$ parametrizes the irreducible representations of $H_{n}$, too. Let $\left(\rho_{\lambda}, V_{\lambda}\right)$ be the representation of $H_{n}$ parametrized by a partition $\lambda \in \Lambda(n)$ and $\chi_{\lambda}$ its character. For each $\lambda \in \Lambda(n)$, we know [12] the representation matrices of $\rho_{\lambda}\left(T_{i}\right)(1 \leq i \leq n-1)$ with respect to a basis parametrized by the standard tableaus corresponding to the partition $\lambda$.

REMARK 3.4. Fix $\lambda \in \Lambda(n)$, and let $\mathcal{S}_{\lambda}$ be the set of standard tableaus corresponding to the partition $\lambda$. For $S \in \mathcal{S}_{\lambda}, S^{*}$ denote the transpose of $S$, which is a standard tableau corresponding to $\lambda^{*}$. Let $\left\{e_{S} \mid S \in \mathcal{S}_{\lambda}\right\}$ and $\left\{e_{S^{*}} \mid S \in \mathcal{S}_{\lambda}\right\}$ be the basis of $V_{\lambda}$ and $V_{\lambda^{*}}$ given in [12] respectively. Let $\eta: V_{\lambda} \rightarrow$ $V_{\lambda^{*}}$ be the linear isomorphism defined by $\eta\left(e_{S}\right)=e_{S^{*}}\left(S \in \mathcal{S}_{\lambda}\right)$. Then there is a diagonal matrix $D$ with respect to the basis $\left\{e_{S} \mid S \in \mathcal{S}_{\lambda}\right\}$ such that

$$
\rho_{\lambda}\left(T_{i}\right)(q)=D^{-1} \eta^{-1}\left(-q \rho_{\lambda} *\left(T_{i}\right)\left(q^{-1}\right)\right) \eta D .
$$

By the defining relations of $B_{n}$ and $H_{n}$, there is an algebra homomorphism $p_{n}^{(\alpha)}: C B_{n} \rightarrow H_{n}$ defined by $p_{n}^{(\alpha)}\left(\sigma_{i}\right)=\alpha T_{i}$.

Theorem $3.5([7])$. Let $l=(-q)^{1 / 2} \alpha, m=(-1)^{1 / 2}\left(q^{-1 / 2}-q^{1 / 2}\right)$ and $P(\cdot)(l, m)$ be the two-variable Jones polynomial. Then, for $b \in B_{n}$, we have 


$$
P\left(b^{\wedge}\right)=\sum_{\lambda \in \Lambda(n)} a_{\lambda}(P) \chi_{\lambda}\left(p_{n}^{(a)}(b)\right),
$$

where the coefficients $a_{\lambda}(P) \in C(\lambda \in \Lambda(n))$ are given in [10] or [15].

The coefficients $a_{\lambda}(P)(\lambda \in \Lambda(n))$ satisfies

$$
a_{\lambda}(P)(\alpha, q)=a_{\lambda^{*}}(P)\left(-\alpha q, q^{-1}\right) .
$$

Let $K$ be a link. For a positive integer $r$ and $\nu \in \Lambda(r)$, let $P^{(r, \nu)}$ be the invariant parametrized by $\left(r, \rho_{\nu}\right)$ as in Section 1.5. Then Remark 3.4 and (3.6) implies the following.

$$
P^{(r, \nu)}(K)(\alpha, q)=P^{(r, \nu *)}(K)\left(-\alpha q, q^{-1}\right) .
$$

Let $I$ be a subset of $\{1, \cdots, n-1\}, H_{I}$ the subalgebra of $H_{n}$ generated by $\left\{T_{i} \mid i \in I\right\}$, and $S_{I}$ the subgroup of $S_{n}$ generated by $\{(i i+1) \mid i \in I\}$. Let $\rho_{\lambda}^{\prime}$ be the representation of $S_{n}$ parametrized by $\lambda \in \Lambda(n)$. Let $\left\{\lambda_{1}, \lambda_{1}+\lambda_{2}, \cdots, \lambda_{1}+\right.$ $\left.\lambda_{2}+\cdots+\lambda_{p}\right\}=\{1,2, \cdots, n\} \backslash I$ and $\Lambda_{I}=\Lambda\left(\lambda_{1}\right) \times \Lambda\left(\lambda_{2}\right) \times \cdots \times \Lambda\left(\lambda_{p}\right)$. Then the irreducible representations of $H_{I}$ and $S_{I}$ are parametrized by $\Lambda_{I}$. The restriction $\left.\rho_{\lambda}\right|_{H_{I}}$ and $\left.\rho_{\lambda}^{\prime}\right|_{s_{I}}$ are sums of irreducible representations of $H_{I}$ and $S_{I}$ respectively, e.g. $\left.\rho_{\lambda}\right|_{H_{I}}=\bigoplus_{\mu \in \Lambda_{I}} m_{\lambda, \mu} \rho_{\mu}$ and $\left.\rho_{\lambda}^{\prime}\right|_{H_{I}}=\bigoplus_{\mu \in \Lambda_{I}} m_{\lambda, \mu}^{\prime} \rho_{\mu}^{\prime}$, where $\rho_{\mu}$ and $\rho_{\mu}^{\prime}$ are representation of $H_{I}$ and $S_{I}$ parametrized by $\mu \in \Lambda_{I}$.

Proposition 3.8. Let $\mu_{0} \in \Lambda_{I}$ such that the corresponding representation $\rho_{\mu_{0}}^{\prime}$ of $S_{b}$ is trivial. Then the above multiplicities $m_{\lambda, \mu_{0}}$ and $m_{\lambda, \mu_{0}}^{\prime}$ are equal.

Proof. The construction of each irreducible representations of $H_{n}$ and $H_{I}$ in [12] satisfies the following: The entries of the matrices of the generators $T_{i}$ are all rational functions of the parameter $q$ with poles at roots of unity. On the other hand, the above proposition is proved for infinitely many integer values of $q$ by using [5] Theorem 7.2. But we know that $\boldsymbol{C} S_{I}$ and $H_{I}$ are semisimple if $q$ is not equal to any roots of unity. Hence $m_{\lambda, \mu_{0}}$ and $m_{\lambda, \mu_{0}}^{\prime}$ are equal if $q$ is not equal to any roots of unity.

REMARK 3.9. Because of the above proposition, we can calculate $m_{\lambda, \mu_{0}}$ by the Littlewood-Richardson rule ([13], 2.8.13).

Let $\rho_{\lambda}$ be the representation of $H_{r n}$ parametrized by $\lambda \in \Lambda(r n)$ and $\rho_{\nu}$ that of $H_{r}$ parametrized by $\nu \in \Lambda(r)$. Let $\pi_{\lambda, \nu}$ be the representation of $B_{r n}$ parametrized by $\rho_{\lambda}$ and $\rho_{\nu}$ as in Section 1.4. In Table 2 , the representation matrices of $\pi_{\lambda, \nu}$ are given in the case of $n=3, r=2$ and $\nu=(2)$. By using Theorems 1.4.10, 1.5.1, 3.5 and (3.7), we may calculate $P^{(2,(2))}, P^{(2,(11))}$ and $P^{(2)}$ for knots equivalent to the closures of 3-braids. To obtain these matrices, we use the representation matrices of the generators of $H_{6}$ given by $W$-graphs introduced in [18]. The $W$-graphs correspond,ng to the irreducible representations of $H_{6}$ are actually 
Table 2

$$
\begin{aligned}
& \nu_{0}=(2) \\
& \lambda=(6) \quad \pi_{\lambda, \nu_{0}}\left(\sigma_{1}\right)=\pi_{\lambda, \nu_{0}}\left(\sigma_{2}\right)=\alpha^{2} q^{2} \text {, } \\
& \lambda=(51) \quad \pi_{\lambda, \nu_{0}}\left(\sigma_{1}\right)=\alpha^{2}\left(\begin{array}{rr}
-1 & 0 \\
b & q^{2}
\end{array}\right), \quad \pi_{\lambda, \nu_{0}}\left(\sigma_{2}\right)=\alpha^{2}\left(\begin{array}{rc}
-1 & q \\
0 & q^{2}
\end{array}\right) \text {, } \\
& \lambda=(42) \quad \pi_{\lambda, \nu_{0}}\left(\sigma_{1}\right)=\alpha^{2}\left(\begin{array}{ccc}
1 / q & 0 & 0 \\
-1 / \sqrt{q} & -1 & 0 \\
1 & q \sqrt{q} & q^{2}
\end{array}\right), \quad \pi_{\lambda, \nu_{0}}\left(\sigma_{2}\right)=\alpha^{2}\left(\begin{array}{ccc}
q^{2} & q \sqrt{q} & 1 \\
0 & -1 & -1 / \sqrt{q} \\
0 & 0 & 1 / q
\end{array}\right), \\
& \lambda=(411) \quad \pi_{\lambda, \nu_{0}}\left(\sigma_{1}\right)=\pi_{\lambda, \nu_{0}}\left(\sigma_{2}\right)=-\alpha^{2} \text {, } \\
& \lambda=(33) \quad \pi_{\lambda, \nu_{0}}\left(\sigma_{1}\right)=\pi_{\lambda, \nu_{0}}\left(\sigma_{2}\right)=-\alpha^{2} \text {, } \\
& \lambda=(321) \quad \pi_{\lambda, \nu_{0}}\left(\sigma_{1}\right)=\alpha^{2}\left(\begin{array}{cc}
1 / q & 0 \\
-1 / \sqrt{q} & -1
\end{array}\right), \quad \pi_{\lambda, \nu_{0}}\left(\sigma_{1}\right)=\alpha^{2}\left(\begin{array}{cc}
-1 & -1 / \sqrt{q} \\
0 & 1 / q
\end{array}\right), \\
& \lambda=(222) \quad \pi_{\lambda, \nu_{0}}\left(\sigma_{1}\right)=\pi_{\lambda, \nu_{0}}\left(\sigma_{2}\right)=\alpha^{2} / q, \\
& \lambda=\text { (3111), (2211), (21111), (111111) } \operatorname{dim} V_{\lambda, \nu_{0}}=0 \text {. }
\end{aligned}
$$

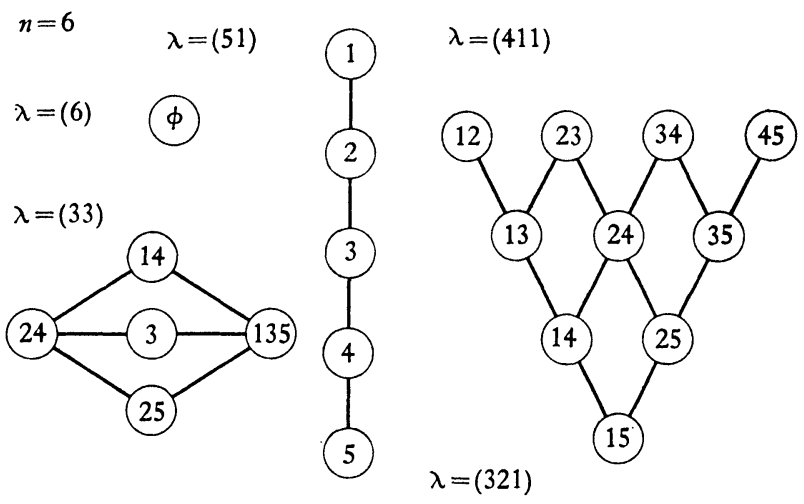

$\lambda=(42)$
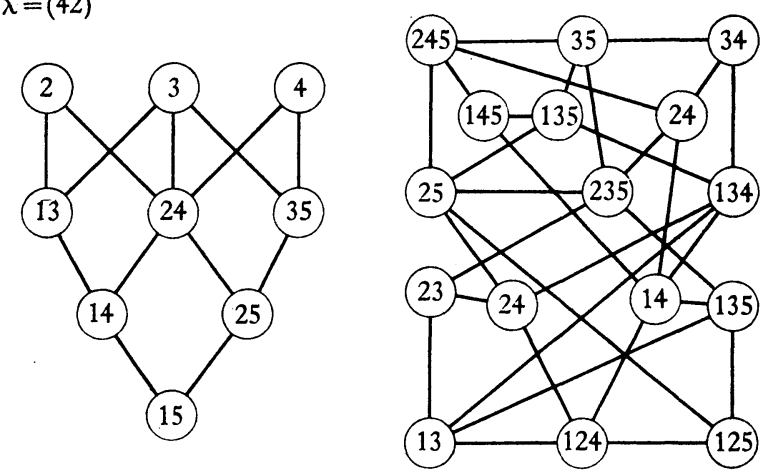

Figure 8

constructed by Naruse and Gyoja (Figure 8).

4. The $r$-parallel version of the one-variable jones polynomial. In this section we discuss the $r$-parallel version $V^{(r)}$ of the one-variable Jones 
polynomial $V$ in detail. We give a formula for the one-variable Jones polynomials of satellite links (Theorem 4.3.2). Let $V^{(r, v)}$ denote the invariant associated with $\nu \in A_{r}(V)^{\wedge}$. The representation matrices of $\pi_{\mu, \nu} \in B_{3}^{\wedge}$ $\left(\mu \in A_{3 r}(V)^{\wedge}, \nu \in A_{r}(V)^{\wedge}\right)$ associated with $V^{(r, \nu)}$ are gives given explicitly (Theorem 4.4.1). By using these matrices we can easily compute $V^{(r)}\left(b^{\wedge}\right)$ for a knot $b^{\wedge}$ which is the closure of a 3-braid $b$. The cases $r=2$ and $r=3$ are discussed closely in (4.4.3)-(4.4.10).

4.1. The jones algebra. In this section we review the definition of the Jones algebra $J_{n}$, which is the associated algebra $A_{n}(V)$ (Definition 1.1.6) of the one-variable Jones polynomial $V$. We also review the construction of the irreducible representations of $J_{n}[15]$ in terms of rectangular diagrams for our later convenience. Fix a non-zero complex number $s$ which is not equal to any root of unity. Let $t^{k / 4}=s^{k}$ for an integer $k$.

Definition 4.1.1. The Jones algebra $J_{n}=J_{n}(t)$ is a $\boldsymbol{C}$-albebra with 1 defined by the following.

$$
\begin{gathered}
J_{n}(t)=\left\langle e_{1}, e_{2}, \cdots, e_{n-1}\right| e_{i} e_{i}=-\left(t^{1 / 2}+t^{-1 / 2}\right) e_{i} \quad(1 \leq i \leq n-1), \\
e_{i} e_{i+1} e_{i}=e_{i}, \quad e_{i+1} e_{i} e_{i+1}=e_{i+1} \quad(1 \leq i \leq n-2), \\
\left.e_{i} e_{j}=e_{j} e_{i} \quad(1 \leq i<j-1 n-1)\right\rangle .
\end{gathered}
$$

For simplicity, we denote $J_{n}(t)$ by $J_{n}$ if there is no fear of confusion.

Since $-\left(t^{1 / 2}+t^{-1 / 2}\right)^{-1} e_{i}(1 \leq i \leq n-1)$ is an idempotent of $J_{n}$, we have

Proposition 4.1.2. For $x \in J, e_{i} x=c x(c \in C \backslash\{0\})$ iff $x \in e_{i} J_{n}$. In this case, $c$ is always equal to $-\left(t^{1 / 2}+t^{-1 / 2}\right)$.

Definition 4.1.3. Let $s$ be a positive real number and $R=[0, s] \times[0,1]$. Let $n$ be a positive integer, $R \ni \alpha_{i}=(0, i /(n+1)), \beta_{i}=(s, i /(n+1))(1 \leq i \leq n)$ and $\gamma_{1}, \cdots, \gamma_{n}$ curves contained in $R$. Then $\left(R,\left\{\gamma_{1}, \cdots, \gamma_{n}\right\}\right)$ is called an (unoriented) rectangular diagram of degree $n$ if it satisfies the following.

(i) Any of the points $\alpha_{1}, \cdots, \alpha_{n}, \beta_{1}, \cdots, \beta_{n}$ is one of the end points of the curves $\gamma_{1}, \cdots, \gamma_{n}$.

(ii) There is no triple crossing point of the curves.

(iii) Every curve has a marking at the each crossing point indicating whether the curve under consideration is the over path or the under path at the crossing.

(iv) The intersection of the curves $\gamma_{1}, \cdots, \gamma_{n}$ and the boundary of $R$ is equal to $\left\{\alpha_{1}, \cdots, \alpha_{n}, \beta_{1}, \cdots, \beta_{n}\right\}$.

A diagram $\left(R,\left\{\gamma_{1}, \cdots, \gamma_{n}\right\}\right)$ is called a rectangualr diagram without crossing points of degree $n$ if the curves $\gamma_{1}, \cdots, \gamma_{n}$ do not intersect themselves. If orientations 
are given to all the curves in $R$, then $R$ is called an oriented rectangular diagram of degree $n$.

We call $\alpha_{1}, \cdots, \alpha_{n}$ (respectively $\beta_{1}, \cdots, \beta_{n}$ ) the points at the top (respectively bottom) of $R$. Two rectangular diagrams $\left([0, s] \times[0,1],\left\{\gamma_{1}, \cdots, \gamma_{n}\right\}\right)$ and $\left(\left[0, s^{\prime}\right] \times[0,1],\left\{\gamma_{1}^{\prime}, \cdots, \gamma_{n}^{\prime}\right\}\right)$ of degree $n$ are called equivalent if there is a homeomorphism $f:[0, s] \times[0,1] \rightarrow\left[0, s^{\prime}\right] \times[0,1]$ such that $f((0, t))=(0, t), f((s, t))=$ $\left(s^{\prime}, t\right)(t \in[0,1])$ and $f\left(\gamma_{i}\right)=\gamma_{i}^{\prime}(1 \leq i \leq n)$.

We define algebras $D_{n}(n \in \boldsymbol{N})$ over $\boldsymbol{C}$. Let $E_{n}^{\prime}$ denote the set of the equivalence classes of rectangular diagrams aithout crossing points of degree $n$. With the convention $\sharp E_{0}^{\prime}=1$, the number $\# E_{n}^{\prime}$ of elements of $E_{n}^{\prime}(n \in \boldsymbol{N})$ satisfy the recursive relations $\# E_{n}^{\prime}=\sum_{k=0}^{n-1} \# E_{k}^{\prime} \# E_{n-k-1}^{\prime}$. Hence $\# E_{n}^{\prime}$ is equal to the Catalan number $\left(\begin{array}{c}2 n \\ n\end{array}\right) /(n+1)([20], \S 2.3 .4 .4)$. As a $C$-vector space, we put $D_{n}=\bigotimes_{e \in \mathbb{B}_{n}^{\prime}} C e ;$ in particular,

$$
\operatorname{dim}_{C} D_{n}=\left(\begin{array}{c}
2 m \\
n
\end{array}\right) /(n+1)
$$

To define the multiplication in $D_{n}$, it is enough to define the product $a b$ for two equivalence classes $a$ and $b$ of rectangular diagrams $\left(\left[0, s\left[\times[0,1],\left\{\gamma_{1}, \cdots, \gamma_{n}\right\}\right)\right.\right.$ and $\left(\left[0, s^{\prime}\right] \times[0,1],\left\{\gamma_{1}^{\prime}, \cdots, \gamma_{n}^{\prime}\right\}\right)$. This is done similarly as in the case of braids by the following rule.

(a) Let $R=\left[0, s+s^{\prime}\right] \times[0,1]$. Let $g:[0, s] \times[0,1] \rightarrow R$ and $g^{\prime}:\left[0, s^{\prime}\right] \times$ $[0,1] \rightarrow R$ be the mappings defined by $g((x, y))=(x, y)$ and $g^{\prime}((x, y))$ $=(x+s, y)$ respectively.

(b) Let $\left(\bigcup_{i=1}^{m} \delta_{i}\right) \cup\left(\bigcup_{i=1}^{n} \gamma_{i}^{\prime \prime}\right)$ be the decomposition of $\left(\bigcup_{i=1}^{n} g\left(\gamma_{i}\right)\right) \cup\left(\bigcup_{i=1}^{n} g^{\prime}\left(\gamma_{i}^{\prime}\right)\right)$ into connected components, where $\delta_{i}(1 \leq i \leq m)$ are closed curves and $\gamma_{i}^{\prime \prime}(1 \leq i \leq n)$ are curves with end points.

(c) Let $d$ be the equivalence class of the rectangular diagram $\left(R,\left\{\gamma_{1}^{\prime \prime}, \cdots\right.\right.$, $\left.\left.\gamma_{n}^{\prime \prime}\right\}\right)$. Then $a b=\left(-t^{1 / 2}-t^{-1 / 2}\right)^{m} d$.

Let $e_{i}^{\prime}(1 \leq i \leq n-1)$ denote the rectangular diagrams given in Figure 9. Then we have

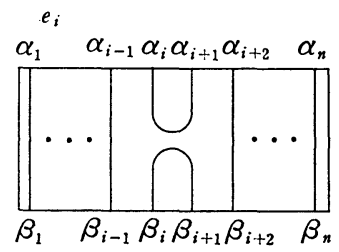

Figure 9 


$$
\begin{array}{ll}
e_{i}^{\prime 2}=\left(-t^{1 / 2}-t^{-1 / 2}\right) e_{i}^{\prime} & (1 \leq i \leq n-1) \\
e_{i}^{\prime} e_{i+1}^{\prime} e_{i}^{\prime}=e_{i}^{\prime}, \quad e_{i+1}^{\prime} e_{i}^{\prime} e_{i+1}^{\prime}=e_{i+1}^{\prime} & (1 \leq i \leq n-2),
\end{array}
$$

Hence there is a homomorphism $\eta$ from $J_{n}$ to $D_{n}$ sending $e_{i}$ to $e_{i}^{\prime}$. But, by [15] and (4.1.4), we have

$$
\operatorname{dim}_{C} J_{n}=\left(\begin{array}{c}
2 n \\
n
\end{array}\right) /(n+1)=\operatorname{dim}_{C} D_{n}
$$

Hence $\eta$ is an siomorphism. Let $E_{n}=\eta^{-1}\left(E_{n}^{\prime}\right)$. Then $E_{n}$ is a basis of $J_{n}$. The definition of the multiplication in $D_{n}$ implies that, for $e_{i}^{\prime}$ and $x \in E_{n}^{\prime}, e_{i}^{\prime} x$ is a nonzero scalar multiple of an element of $E_{n}^{\prime}$. Hence $e_{i} J_{n}$ is spanned by a subset of $E_{n}$. Combining this observation with Proposition 4.1.2, we get the following:

Proposition 4.1.6. The subspace $e_{i} J_{n}$ of $J_{n}$ is spanned by $E_{n}^{(i)}=\left\{\ell^{-1}(x) \mid\right.$ $x \in E_{n}^{\prime}$, the $i$-th and $(i+1)$-th points at the top of $x$ are connected by a curve of $\left.x\right\}$.

For $y \in E_{n}^{\prime}$, let $T_{D}(y)$ denote the number of strings of $y$ connecting two points at the top of $y$. For example,

$$
T_{D}\left(\eta\left(e_{1} e_{3} \cdots e_{2 j-1}\right)\right)=j \text {. }
$$

Let $E_{n, i}=\left\{\eta^{-1}(y) \mid y \in E_{n}^{\prime}, T_{D}(y) \geq i\right\}$ and $J_{n, 1}^{\cdot}$ the two-sided ideal of $J_{n}$ generated by the elements of $E_{n, i}$. Since $T_{D}\left(y y^{\prime}\right) \geq \max \left(T_{D}(y), T_{D}\left(y^{\prime}\right)\right)$ for $y, y^{\prime} \in E_{n}, J_{n, i}^{\cdot}$ is equal to the $\boldsymbol{C}$-linear span of $E_{n, i}$ in $J_{n}$. For $x \in J_{n}$, we have

$$
e_{i(1)} e_{i(2)} \cdots e_{i(k)} x \in J_{n, k}^{\cdot} \quad(i(j)<i(j+1)-1 \text { for } 1 \leq j \leq k) .
$$

Let $J_{n, i}=J_{n, i}^{\bullet} / J_{n, i+i}^{\bullet}$. Since $J_{n}$ is known to be semisimple, the canonical projection $J_{n, i}^{:} \rightarrow J_{n, i}$ splits. Hence $J_{n, i}$ can be regarded as a two-sided ideal of $J_{n}$.

Proposition 4.1.9 The two-sided ideals $J_{n, i}(0 \leq i \leq[n / 2])$ of $J_{n}$ are simple algebras and $J_{n} \simeq \bigoplus_{i=0}^{[n / 2]} J_{n, i}$.

Proof. Let $e(1,2 i-1)=e_{1} e_{3} \cdots e_{2 i-1}(0 \leq i \leq[n / 2])$. Since $e(1,2 i-1) \in J_{n, i}^{*}$ and $e(1,2 i-1) \notin J_{n, i+1}^{*}, J_{n, i} \neq\{0\}$ for $0 \leq i \leq[n / 2]$. We also know ([15], §11) that $J_{n}$ is a direct sum of $[n / 2]+1$ two-sided ideals which are simple algebras. Hence, by using Jordan-Holder's Theorem, we conclude that the two-sided ideals $J_{n, i}(0 \leq i \leq[n / 2])$ are non-trivial simple algebras.

Since $J_{n}$ is a semisimple algebra over $\boldsymbol{C}$, the two-sided ideals $J_{n i}$ of $J_{n}(0 \leq$ $i \leq[n / 2])$ are isomorphic to the full matrix algebras over $\boldsymbol{C}$. Let $\rho_{n, i}: J_{n} \rightarrow J_{n, i}$ denote the associated irreducible representations of $J_{n}$, and $\chi_{n, i}$ their charactews.

Let $p_{n}, p_{n}^{\prime}: C B_{n} \rightarrow J_{n}(t)$ be the algebra homomorphisms defined by $\sigma_{i} \rightarrow$ $-t^{1 / 2}-t e_{i}$ and $\sigma_{i} \rightarrow t^{-1 / 4}+t^{1 / 4} e_{i}$ respectively. Then the Jones polynomial $V$ and 
the bracket polynomial $\langle\cdot\rangle$ for the closure of an $n$-braid $b$ are written as linear combinations of these characters, i.e.

$$
\begin{aligned}
& V\left(b^{\wedge}\right)=\sum_{i=0}^{[r / 2]} a_{n, i}(V) \chi_{n, i}\left(p_{n}(b)\right), \\
& \left\langle\left|b^{\wedge}\right|\right\rangle=\sum_{i=0}^{[r / 2]} a_{n, i}(V) \chi_{n, i}\left(p_{n}^{\prime}(b)\right) .
\end{aligned}
$$

The coefficients $a_{n, i}(V)$ are given by

$$
a_{n, i}(V)=(-1)^{n+1}\left(t^{n / 2+1 / 2-i}-t^{-n / 2-1 / 2+i}\right)\left(t-t^{-1}\right),
$$

(see [10], [15], [16]).

Note that none of the coefficients $a_{n, i}(V)$ are equal to zero because $t$ is not a root of unity.

REMARK 4.1.12. Let $\varphi_{n}: H_{n}(t) \rightarrow J_{n}(t)$ be the algebra homomorphism defined by $\varphi_{n}\left(T_{i}\right)=-1-t^{1 / 2} e_{i}$. The compositions $\rho_{n, i} \circ \varphi_{n}$ for $0 \leq i \leq[n / 2]$ give irreducible representations of $H_{n}(t)$ parametrized by the partitions $(22 \cdots 211 \cdots 1)$.

$$
i \text {-time }(n-2 i) \text {-time }
$$

Definition 4.1.13. Let $G_{n}$ denote the free semigriup generated by $1, f_{i}$, $\sigma_{i}, \sigma_{i}^{-1}(1 \leq i \leq n-1), G=\left\{(g, n) \mid g \in G_{n}\right\}$ and for $(g, n) \in G$, let $d(g, n)$ denote the rectangular diagram (Definition 4.1.3) of $(g, n)$ defined as in Figure 10.
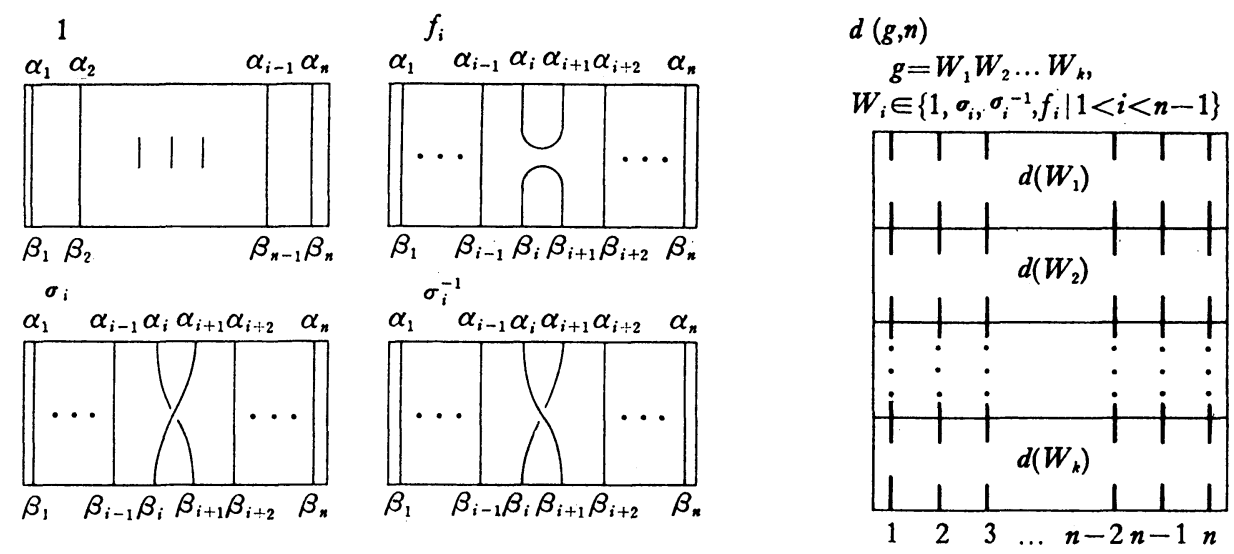

Figure 10

By the analogy with braids we use the term closure of a rectangular diagram $d(g, n)$, written $(g, n)^{\wedge}$, to mean the link formed by joining the $n$ points at the top of $d(g, n)$ to those at the bottom without further crossings. Let $\Psi_{n}: C G_{n} \rightarrow J_{n}$ denote the algebra homomorphism defined by 


$$
\Psi_{n}\left(f_{i}\right)=e_{i}, \quad \Psi_{n}\left(\sigma_{i}\right)=t^{-1 / 4}+t^{1 / 4} e_{i}, \quad \Psi_{n}\left(\sigma_{i}^{-1}\right)=t^{1 / 4}+t^{-1 / 4} e_{i} .
$$

By using the original definition of $\langle\cdot\rangle$ in [16], we can show that

$$
\left\langle(g, n)^{\wedge}\right\rangle=\sum_{i=0}^{[r / 2]} a_{n, i}(V) \chi_{n, i}\left(\Psi_{n}(g)\right),
$$

where the coefficients $a_{n, i}(V)$ are given by (4.1.11).

4.2. Relations among the invariants associated with the decomposition (1.5.1) of $\boldsymbol{V}^{(r)}$. Let $p_{r}$ be the homomorphism from $C B_{r}$ to $J_{r}$ defined just before (4.1.10). Let $V^{(r, i)}$ denote the invariant associated with the irreducible representation $\rho_{r, i}{ }^{\circ} p_{r}$ of $B_{r}$ (see Sections 1.4 and 4.1). In this section, we give relations among $V^{(r, i)}$. In the following we use the conventions that $V^{(0)}(K)=V^{(0,0)}(K)=\langle|K|\rangle^{(0)}=\langle|K|\rangle^{(0,0)}(K)=\left(-t^{-1 / 2}-t^{1 / 2}\right)^{-1}$ for any link diagram $K$.

Theorem 4.2.1. Let $K$ be a knot. Then we have

$$
V^{(r, i)}(K)=V^{(r-2 k, i-k)}(K) \quad \text { for } \quad r \geq 0,0 \leq i \leq[r / 2], 0 \leq k \leq i .
$$

Remark 4.2.2. The same is also true in the case when $K$ is a general link. We restrict our attention to the present case for simplicity.

Corollary 4.2.3. Let $K$ be a knot. Then we have

(i) $\quad V^{(r)}(K)=\sum_{i=0}^{[r / 2]} \chi_{r, i}(1) V^{(r-2 i, 0)}(K)$ for $r \geq 0$,

(ii) $\quad V^{(r, 0)}(K)$ is a linear combination of $V^{(s)}(K)(s=r, r-2, \cdots, r-2[r / 2])$ with integer coefficients.

(iii) $\left(t^{1 / 2}+t^{-1 / 2}\right) V^{(r, j)}(K)$ is a Laurent polynomial in the parameter $t^{1 / 2}$.

Proof. The formula (i) is an immediate consequence of Theorems 1.4.10 and 4.2.1. Solving the formula (i) with respect to $V^{(s, 0)}(K)$, we get

$$
V^{(s, 0)}(K)=\sum_{r=s, s-2, \ldots} c_{s, r} V^{(r)}(K) \quad\left(c_{s, r} \in \boldsymbol{C}\right) .
$$

Since $\chi_{r, 0}(1)=1$, the coefficients $c_{s, r}(r=s, s-2, \cdots)$ are all integers and so we get (ii). By (4.1.11), the coefficients $\left(t^{1 / 2}+t^{-1 / 2}\right) a_{r n, i}(V)$ are Laurent polynomials in $t^{1 / 2}$. We also know [12] that the characters $\chi_{r n, i}\left(p_{r n}(b)\right)(n \in N, 0 \leq i \leq[r n / 2]$, $b \in B_{r n}$ ) are Laurent polynomials in $t^{1 / 2}$. Hence (ii) follows from (iii).

To prove Theorem 4.2.1, we need the bracket polynomial $\langle\cdot\rangle$ defined on $G$ (see the last paragraph of Section 4.1). By (4.1.10), the bracket polynomial is of trace type. Applying Theorem 1.6.4 to $\langle\cdot\rangle$, we get the following.

Proposition 4.2.4. For $b^{\prime} \in C B_{r}$ and a braid $(b, n)$ we have

$$
\left\langle(b, n)^{\wedge}\right\rangle_{b^{\prime}}^{(r)}=\sum_{i=0}^{[r / 2]} \chi_{r, i}\left(p_{r}^{\prime}\left(b^{\prime}\right)\right)\langle(b, n)\rangle^{(r, i)} \quad \text { for } \quad r \geq 0 .
$$


Let $f_{i}^{\prime} \in C B_{r n}$ such that $p_{r n}^{\prime}\left(f_{i}^{\prime}\right)=e_{i}(1 \leq i \leq r n-1)$. Then we have the following :

Proposition 4.2.5. For $b \in B_{n}$, we have

$$
\langle(b, n)\rangle_{f_{1}^{\prime} f_{3}^{\prime} \cdots f_{2 j-1}^{\prime}}^{\prime}=\left(-t^{3 / 4}\right)^{-2 j w\left(b^{\wedge}\right)}\left(-t^{1 / 2}-t^{-1 / 2}\right)^{j}\langle(b, n)\rangle^{r-2 j}
$$

Proof. For $g \in G_{r n}$, we put $\langle g\rangle=\left\langle d(g, n)^{\wedge}\right\rangle \in C$. By Definition 1.1 .10 of the bracket polynomial, the mapping $\langle\cdot\rangle: G_{r n} \rightarrow C$ is factored by the projection $\Psi_{r n}: G_{r n} \rightarrow J_{r n}$ (see the last paragraph of Section 4.1). Since $p_{r n}^{\prime}\left(f_{1}^{\prime} f_{3}^{\prime}, \cdots, f_{2 j-1}^{\prime}\right)=$ $\Psi_{r n}\left(f_{1} f_{3} \cdots f_{2_{j-1}}^{\prime}\right)$, we have

$$
\begin{aligned}
\langle(b, n)\rangle_{f_{1}^{\prime} f_{3}^{\prime} \cdots f_{2 j-1}^{\prime}}^{\prime} & =\left\langle\left(f_{1}^{\prime} f_{3}^{\prime} \cdots f_{2 j-1}^{\prime} \phi_{n}^{(r)}(b), r n\right)\right\rangle \\
& =\left\langle\left(f_{1} f_{3} \cdots f_{2 j-1} \phi_{n}^{(r)}(b), r n\right)\right\rangle .
\end{aligned}
$$

The following lemma shows that the link diagram $\left(f_{1} f_{3} \cdots f_{2 j-1} \phi_{n}^{(r)}(b), r n\right)^{\wedge}$ is regular isotopic to

$$
\left(\phi_{n}^{(r-j)}(b),(r-2 j) n\right)^{\wedge} \cup\left(\cup_{i=1}^{j}\left(f_{1} \sigma_{1}^{-2 w\left(b^{\wedge}\right)}, 2\right)^{\wedge}\right) \quad \text { (disjoint union) . }
$$

For a link diagram $K$ which is a disjoint union of two link diagrams $K_{1}$ and $K_{2}$, it is known that $\langle K\rangle=\left(-t^{1 / 2}-t^{-1 / 2}\right)\left\langle K_{1}\right\rangle\left\langle K_{2}\right\rangle$. It is also known ([16], Proposition 2.5) that $\left\langle\left(f_{1} \sigma_{1}^{-2 w\left(b^{\wedge}\right)}, 2\right)\right\rangle=\left(-t^{3 / 4}\right)^{-2 w\left(b^{\wedge}\right)}$. Hence we have

$$
\begin{aligned}
& \left.\left\langle\left(f_{1} f_{3} \cdots f_{2 j-1} \phi_{n}^{(r)}\right)(b), r n\right)^{\wedge}\right\rangle \\
& \quad=\left\langle\phi_{n}^{(r-j)}((b),(r-2 j) n)^{\wedge} \cup\left(\bigcup_{i=1, \cdots, j}\left(f_{1} \sigma_{1}^{-2 w\left(b^{\wedge}\right)}, 2\right)^{\wedge}\right)\right\rangle \\
& \quad=\left(-t^{3 / 4}\right)^{-2 j w\left(b^{\wedge}\right)}\left(-t^{1 / 2}-t^{-1 / 2}\right)^{j}\left\langle\left(\phi_{n}^{(r-j)}(b),(r-2 j) n\right)\right\rangle,
\end{aligned}
$$

as required.

Lemma 4.2.6. For $b \in B_{n}$, the link diagram $\left(f_{1} f_{3} \cdots f_{2 j-1} \phi_{n}^{( \pm)}(b), r n\right)^{\wedge}$ is regular isotopic to the disjoint union of the link diagrams $\left(\phi_{n}^{(r-j)}(b),(r-2 j) n\right)^{\wedge}$ and copies $j$ of $\left(f_{1} \sigma_{1}^{-2 w\left(b^{\wedge}\right)}, 2^{\wedge}\right)$.

Proof. Let $\beta=s_{i(1)}^{(\mathrm{g}(1))} s_{i(2)}^{(\mathrm{q}(2))} \cdots s_{i(k)}^{(\mathrm{g}(k))}$ where $3 \leq i(j) \leq r-1,-1 \leq \varepsilon \leq(j) \leq 1$ $(1 \leq j \leq k), s_{i(j)}^{(0)}=f_{i(j)}, s_{i(j)}^{(1)}=\sigma_{i(j)}$ and $s_{i(j)}^{(-1)}=\sigma_{i(j)}^{-1}$. Let $\beta^{*}=s_{i(1)-2}^{(\mathcal{e}(1))} s_{i(2)-2}^{(\mathrm{e}(2))} \cdots s_{i(k)-2}^{(\mathcal{8}(k))}$. Then Figure 11 demonstrates that the link diagram $\left(f_{2} \beta \phi_{n}^{(r)}(b), r n\right)^{\wedge}$ is regular isotopic to the disjoint union of the link diagrams $\left(\beta^{*} \phi_{n}^{(r-2)}(b),(r-2) n\right)^{\wedge}$ and $\left(f_{1}, \sigma_{1}^{-2 w\left(b^{\wedge}\right)}, 2\right)^{\wedge}$. Hence an induction on $j$ proves the statement of the lemma.

Lemma 4.2.7. Let $e(j)=e_{1} e_{3} \cdots e_{2 j-1} \in J_{r}(1 \leq j \leq[r / 2])$. Let $\chi_{r, i}$ be the character of the representation $\rho_{r, i}$ of $J_{r}$, defined in Section 4.1. We have
(a) $\chi_{r, i}(e(j))=0$
if $i<j$, 

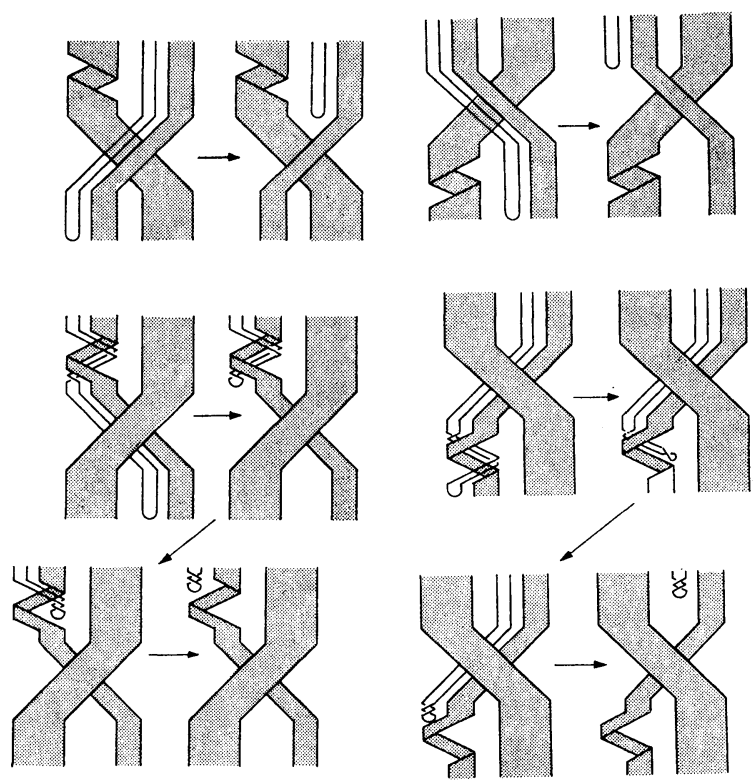

Figure 11

(b) $\chi_{r, i}(e(j))=\left(-t^{1 / 2}-t^{-1 / 2}\right)^{j} \chi_{r-2 j, i-j}(1)$ if $0 \leq j \leq i$, where we used the convention that $x_{0,0}(1)=1$.

Proof. Since $T_{D}(e(j))=j$ by (4.1.7), $\rho_{r, i}(e(j))=0$ if $j<i$ and so we get the first formula of the above lemma. We regard $J_{r-1}$ as a subalgebra of $J_{r}$ by the inclusion homomorphism sending $e_{i} \in J_{r-1}$ to $e_{i} \in J_{r}(1 \leq i \leq r-2)$. For an element $x \in J_{r}$ contained in the subalgebra $J_{r-1}$, we know [15] that

$$
\begin{aligned}
& \chi_{r, 0}(x)=\chi_{r-1,0}(x), \\
& \chi_{r, i}(x)=\chi_{r-1, i}(x)+\chi_{r-1, i-1}(x) \quad \text { if } \quad 0<i<r / 2, \\
& x_{2 r, r}(x)=x_{2 r-1, r-1}(x) .
\end{aligned}
$$

Using (4.2.8) and Lemma 4.2.9 below, we get the part (b) by induction on $r$. The details are omitted.

Lemma 4.2.9. $\chi_{2 r, r}(e(r))=\left(-t^{1 / 2}-t^{-1 / 2}\right)^{r}$.

Proof. By Lemma 4.2.7 (a) and (4.2.8), it is enough to show:

$$
X_{2 r, r}(e(r))=\left(-t^{1 / 2}-t^{-1 / 2}\right) X_{2 r-1, r-1}(e(r-1)) .
$$

Let $e(i)^{\prime}$ be an element of $\boldsymbol{C} B_{2 r}$ with $p_{2 r}\left(e(i)^{\prime}\right)=e(i)(1 \leq i \leq r)$. By Lemma 4.2.7 (a), $\chi_{2 r, i}(e(r))=0$ if $i<r$ and $X_{2 r-1, i}(e(r-1))=0$ if $i<r-1$. Hence we have

$$
V_{2 r}\left(e(r)^{\prime}\right)=a_{2 r, r}(V) \chi_{2 r, r}(e(r))
$$

and 


$$
V_{2 r-1}\left(e(r-1)^{\prime}\right)=a_{2 r-1, r-1}(V) \chi_{2 r-1, r-1}(e(r-1)) .
$$

Since $V$ is a link invariant, we have

$$
V_{2 r}\left(p_{2 r}\left(\sigma_{2 r-1}\right) e(r-1)^{\prime}\right)=V_{2 r}\left(p_{2 r}\left(\sigma_{2 r-1}^{-1}\right) e(r-1)^{\prime}\right)=V_{2 r-1}\left(e(r-1)^{\prime}\right)
$$

(Definition 1.1.2 (ii)).

Hence, by using

$$
e_{i}=t^{1 / 2}\left(t^{1 / 2}-t^{-1 / 2}\right)^{-1} p_{2 r}\left(\sigma_{i}\right)-t^{-1 / 2}\left(t^{1 / 2}-t^{-1 / 2}\right)^{-1} p_{2 r}\left(\sigma_{i}^{-1}\right),
$$

we have

$$
\begin{aligned}
& a_{2 r, r}(V) \chi_{2 r, r}(e(r))=V\left(e(r)^{\prime}, 2 r\right) \\
& \quad=V\left(\left(t^{1 / 2}\left(t^{1 / 2}-t^{-1 / 2}\right)^{-1} p_{2 r}\left(\sigma_{2 r-1}\right)-t^{-1 / 2}\left(t^{1 / 2}-t^{-1 / 2}\right)^{-1} p_{2 r}\left(\sigma_{2 r-1}^{-1}\right)\right) e(r-1)^{\prime}, 2 r\right) \\
& \quad=V\left(e(r-1)^{\prime}, 2 r-1\right)=a_{2 r-1, r-1}(V) \chi_{2 r-1, r-1}(e(r-1)) .
\end{aligned}
$$

This and (4.1.11) imply (4.2.10).

Proof of Theorem 4.2.1. From Proposition 4.2.4 and Lemma 4.2.7 we have

$$
\begin{aligned}
& \langle(b, n)\rangle_{f_{1}^{\prime} f_{3}^{\prime} \cdots f_{2 j-1}^{\prime}}^{(r)} \\
& =\left(-t^{1 / 2}-t^{-1 / 2}\right)^{j}\left(\sum_{i=j}^{[r / 2]} \chi_{r-2 j, i-j}(1)\langle(b, n)\rangle^{(r, i)}\right) \quad(0 \leq r, 0 \leq j \leq[r / 2]) .
\end{aligned}
$$

By interchanging $r$ with $r+2 j$ and $i$ with $i+j$, we get

$$
\begin{aligned}
& \langle(b, n)\rangle_{f_{1}^{\prime} f_{3}^{\prime} \cdots f_{2 j-1}^{\prime}}^{(r+2 j)} \\
& =\left(-t^{1 / 2}-t^{-1 / 2}\right)^{j}\left(\sum_{i=0}^{[r / 2]} \chi_{r, i}(1)\langle(b, n)\rangle^{(r+2 j, i+j)}\right) \quad(0 \leq r, 0 \leq j \leq[r / 2]) .
\end{aligned}
$$

Hence Proposition 4.2.5 implies that

$$
\langle(b, n)\rangle^{(r)}=\left(-t^{3 / 4}\right)^{2 j w\left(b^{\wedge}\right)}\left(\sum_{i=0}^{[r / 2]} \chi_{r, i}(1)\langle(b, n)\rangle^{(r+2 j, i+j)} .\right.
$$

By using (1.1.12), we get $V^{(r)}(b, n)=\left(-t^{3 / 4}\right)^{r w\left(b^{\wedge}\right)}\langle(b, n)\rangle^{(r)}$ and $V^{(r, i)}(b, n)=$ $\left(-t^{3 / 4}\right)^{r\left(b^{\wedge}\right)}\langle(b, n)\rangle^{(r, i)}$. Therefore we have

$$
V^{(r)}(b, n)=\sum_{i=0}^{[r / 2]} x_{r, i}(1) V^{(r+2 j, i+j)}(b, n) .
$$

But the left hand side of the above formula does not depend on $j$, and so we obtain inductively that

$$
\begin{aligned}
& V^{(0)}(b, n)=V^{(0,0)}(b, n)=V^{(2,1)}(b, n)=\cdots=V^{(2 i, i)}(b, n)=\cdots, \\
& V^{(1)}(b, n)=V^{(1,0)}(b, n)=V^{(3,1)}(b, n)=\cdots=V^{(1+2 i, i)}(b, n)=\cdots,
\end{aligned}
$$




$$
\begin{aligned}
& V^{(r)}(b, n)-\sum_{[i=1}^{[r / 2} \chi_{r, i}(1) V^{(r, i)}(b, n) \\
& =V^{(r, 0)}(b, n)=V^{(r+2,1)}(b, n)=\cdots=V^{(r+2 j, j)}(b, n)=\cdots,
\end{aligned}
$$

These are the equalities we wanted to show.

4.3. The Jones polynomial of satellite links. We give a satellite link version of Theorem 1.6.4 for the one-variable Jones polynomial $V$.

Definition 4.3.1. Let $K$ and $L$ be diagrams of links in the 3-sphere and the solid torus respectively. Let $N(K)$ the tubular neighborhood of $K$. Then there is a bijection $f$, called the faithful embedding, from the solid torus to $N(K)$. This mapping is determined canonically up to ambient isotopy. Let $K_{L}=f(L)$. Then $K_{L}$ is a link in the 3-sphere and is called the satellite link of $K$ with respect to $L$. For a link invariant $X$, put $X_{L}(K)=X\left(K_{L}\right)$. Then $X_{L}$ is a link invariant.

For a link diagram $L$, let $|L|$ denote the unoriented link diagram obtained by forgetting the orientation of $L$. For $(g, n) \in G$ (Definition 4.1.13), let $(g, n)^{\sim}$ be the unoriented link diagram in the annulus obtained by joining the points at the top of $d(g, n)$ and those at the bottom without further crossings as in Figure 12. Conversely, for any link diagram $L$ in the annulus, there is an element $(g, n) \in G$ such that $|L|$ is regular isotopic to $(g, n)^{\sim}$ in the annulus. Let $\Psi_{r}$ : $G_{r} \rightarrow J_{r}$ be the projection defined in Section 4.1.

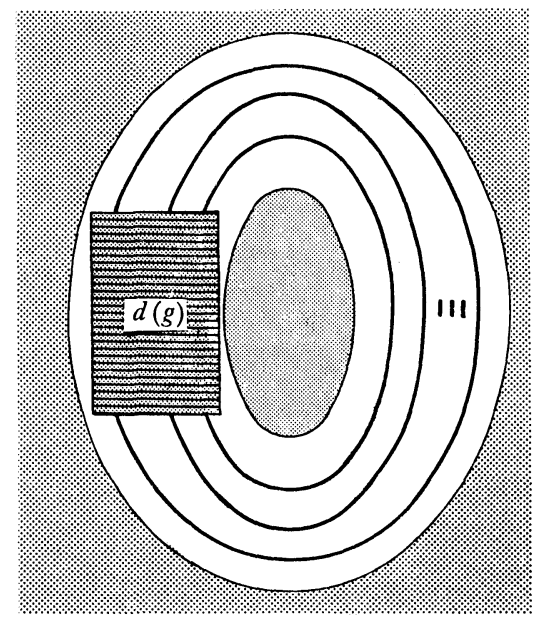

Figure 12

Theorem 4.3.2. Let $K$ be a knot, $L$ an oriented link diagram in the annulus, $w(L)$ the writhe of $L$, and $(g, r)$ an element of $G$ whose closure is regular isotopic to $|L|$ in the annulus. Then we have 


$$
V_{L}(K)=\left(-t^{3 / 4}\right)^{w(L)} \sum_{i=0}^{[r / 2]} \chi_{r, i}\left(\Psi_{r}(g)\right) V^{(r, i)}(K) .
$$

Proof. Let $(b, n) \in B$ be a braid whose closure is equivalent to $K$. Then $K_{L}$ is equivalent to $\left(g \phi_{n}^{(r)}(b), r n\right)^{\wedge}$. We regard $G_{r}$ as a subsemigroup of $G_{r n}$ by the inclusion homomorphism sending $g \in G_{r}$ to the isotopy class of $g^{\prime}$ in $G_{r n}$ as in Figure 13.

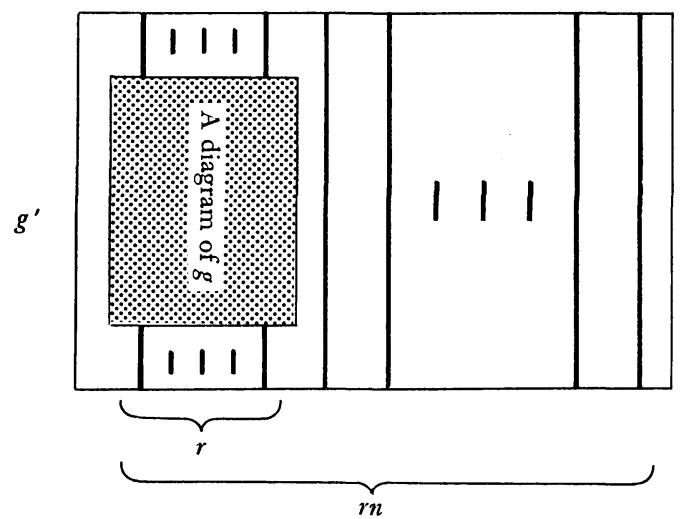

Figure 13

By applying the results of Section 1.3 to the wreath product $G_{n}^{r} \rtimes G_{n}$, we get

$$
\left\langle\left(g \phi_{n}^{(r)}(b), r n\right)\right\rangle=\sum_{i=0}^{[r / 2]} \chi_{r, i}\left(\Psi_{r}(g)\right)\langle(b, n)\rangle^{(r, i)} .
$$

The proof of this formula is analogous to that of Theorem 1.6.4. We know that $V^{(r, i)}(b, n)=\left(-t^{3 / 4}\right)^{w\left(b^{\wedge}\right)}\langle(b, n)\rangle^{(r, i)}$ and $w\left(\left(g \phi_{n}^{(r)}(b), r n\right)^{\wedge}\right)=w\left((g, r)^{\wedge}\right)+r w\left(b^{\wedge}\right)$. By using these formulas, the above statement can be proved.

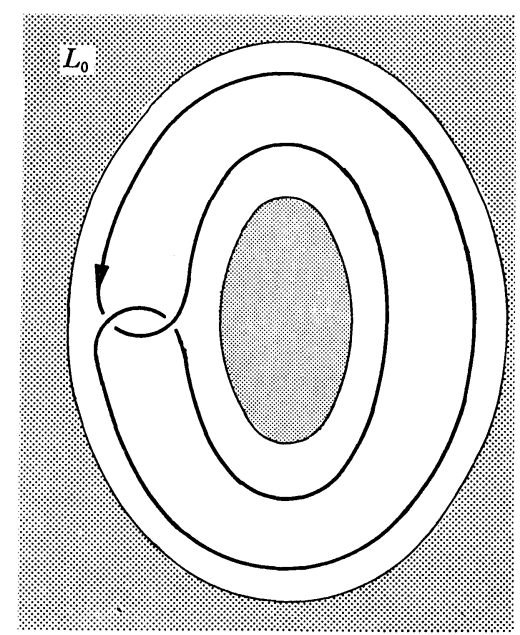

Figure 14 
Theorem 4.3.2 can be extended for marked links as Theorem 2.5.4.

EXAMPle 4.3.3. (doubled knot [33]). Let $L_{0}$ be the link diagram in the annulus as in Figure 14. Then $L_{0}$ is regular isotopic to $\left(g_{0}, 4\right) \sim$, where $g_{0}=$ $f_{1} f_{2} f_{3} \sigma_{2}^{2}$. For a knot $K$, we have $V_{L_{0}}(K)=t^{-3 / 2}\left((1-t) V^{(2,0)}(K)-\left(t^{-1}+t\right) V^{(0,0)}(K)\right)$ since $\chi_{4,0}\left(\Psi_{4}\left(g_{0}\right)\right)=0, \chi_{4,1}\left(\Psi_{4}\left(g_{0}\right)\right)=1-t$ and $\chi_{4,2}\left(\Psi_{4}\left(g_{0}\right)\right)=-\left(t^{-1}+t\right)$. But we know that $V^{(2,0)}(K)=V^{(2)}(K)+\left(t^{-1 / 2}+t^{1 / 2}\right)^{-1}$ from Corollary 4.2.3 and so we have $V_{L_{0}}(K)=t^{-3 / 2}(1-t) V^{(2)}(K)+t^{-2}$.

4.4. Representations associated with $\boldsymbol{V}^{(r)}$. Let $\rho_{m, k}$ denote the irreducible representation of $J_{m}$ defined in (4.1.9)-(4.1.10). In this section, $\pi_{3 r, i}$ $(0 \leq i \leq[3 r / 2])$ denote the representations of $B_{3 r}$ parametrized by two irreducible representations $\rho_{3 r, i}$ of $J_{3 r}$ and $\rho_{r, 0}$ of $J_{r}$ defined as in Section 1.4. Let $\omega_{3 r i}$ denote the character of $\pi_{3 r, i}$. Let $K$ be a knot equivalent to the closure of a 3-braid $b \in B_{3}$. Then, by using the following theorem, (4.1.11) and Theorem 1.4 .10 (ii), we can calculate $V^{(r, 0)}(K)$ explicitly. Moreover, by using Corollary 4.2.3 (i), we may calculate $V^{(r)}(K)$ explicitly.

Theorem 4.4.1. A set of representation matrices of $\pi_{3 r, i}\left(\sigma_{j}\right)(0 \leq i \leq[3 r / 2]$, $j=1,2)$ is given by the following.

$$
\begin{aligned}
& \pi_{3 r, i}\left(\sigma_{1}\right)=\left(\alpha_{i, j, k}(t)\right)_{0 \leq j, k \leq d}(r, i), \\
& \pi_{3 r, i}\left(\sigma_{2}\right)=\left(\alpha_{i, d(r, i)-j, d(r, i)-k}(t)\right)_{0 \leq j, k \leq d(r, i)},
\end{aligned}
$$

where $d(r, i)=\operatorname{deg}\left(\pi_{3 r, i}\right)-1=i($ if $0 \leq i \leq r)$ or $3 r-2 i($ if $r<i \leq[3 r / 2])$ and

$$
\begin{array}{ll}
\alpha_{i, j, k}(t)=(-1)^{(r+k)} t^{\gamma(r, i, k, j)} g(i-k, j-k, t) & (k \leq j, 0 \leq i \leq r), \\
\alpha_{i, j, k}(t)=0 & (j<k, 0 \leq i \leq r), \\
\alpha_{i, j, k}(t)=(-1)^{(r+k)} t^{\gamma(r, 2 r-i, k, j)} g(i-k, j-k, t) & (k \leq j, r<i \leq[3 r / 2]), \\
\alpha_{i, j, k}(t)=0 & (j<k, r<i \leq[3 r / 2]),
\end{array}
$$

where $\left.\gamma(r, p, k, j)=r+k+2 r k-k^{2}+(r-p+j-k)(j-k)\right) / 2$ and $g(p, q, t)$ is the Gauss' polynomial, i.e.

$$
g(p, q, t)=\prod_{i=1}^{q} \frac{t^{p+i-1}-1}{t^{i}-1}
$$

The polynomial $g(p, q, t)$ satisfy the following recursive relations:

$$
g(p, 0, t)=1, \quad g(p, p, t)=1,
$$

$$
g(p, q, t)=t^{(p-q)} g(p-1, q-1, t)+g(p-1, q, t) .
$$

Before proving the above theorem, we give examples and applications. 
Table 3

$$
\begin{aligned}
& r=2 \text { : } \\
& \pi(6,0),(2,0)\left(\sigma_{1}\right)=t, \\
& \pi(6,1),(2,0)\left(\sigma_{1}\right)=\left(\begin{array}{cc}
t & 0 \\
t^{2} & -t^{3}
\end{array}\right), \\
& \pi(6,2),(2,0)\left(\sigma_{1}\right)=\left(\begin{array}{ccc}
t & 0 & 0 \\
t^{3 / 2}(1+t) & -t^{3} & 0 \\
t^{3} & -t^{7 / 2} & t^{4}
\end{array}\right), \\
& \pi(6,3),(2,0)\left(\sigma_{1}\right)=-t^{3}, \\
& r=3 \text { : } \\
& \pi(9,0),(3,0)\left(\sigma_{1}\right)=-t^{3 / 2}, \\
& \pi(9,1),(3,0)\left(\sigma_{1}\right)=\left(\begin{array}{cc}
-t^{3 / 2} & 0 \\
-t^{3} & t^{9 / 2}
\end{array}\right) \text {, } \\
& \pi(9,2),(3,0)\left(\sigma_{1}\right)=\left(\begin{array}{ccc}
-t^{3 / 2} & 0 & 0 \\
-t^{5 / 2}(1+t) & t^{9 / 2} & 0 \\
-t^{3} & t^{11 / 2} & -t^{13 / 2}
\end{array}\right), \\
& \pi(9,3),(3,0)\left(\sigma_{1}\right)=\left(\begin{array}{cccc}
-t^{3 / 2} & 0 & 0 & 0 \\
-t^{2}\left(1+t+t^{2}\right) & t^{9 / 2} & 0 & 0 \\
-t^{7 / 2}\left(1+t+t^{2}\right) & t^{5}(1+t) & -t^{13 / 2} & 0 \\
-t^{6} & t^{13 / 2} & -t^{7} & t^{15 / 2}
\end{array}\right) \text {, } \\
& \pi(9,4),(3,0)\left(\sigma_{1}\right)=\left(\begin{array}{cc}
t^{9 / 2} & 0 \\
t^{11 / 2} & -t^{13 / 2}
\end{array}\right) \text {. }
\end{aligned}
$$

EXAMPLE 4.4.3. The representation matrices of $\sigma_{1} \in B_{3}$ for the case $r=2$ and $r=3$ are given in Table 3. The matrices of $\sigma_{2}$ are obtained as follows.

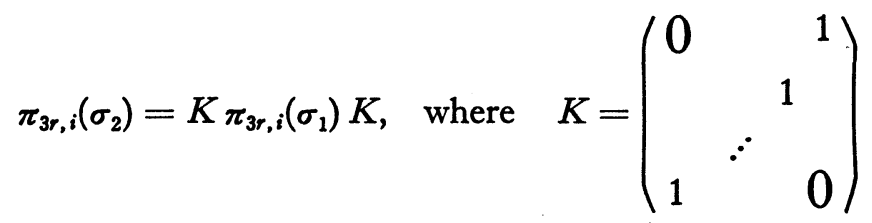

Let $\boldsymbol{C}^{\cdot}=\left\{c \in C \mid c \neq 0, c^{m} \neq 1\right.$ for any $\left.m \in \boldsymbol{N}\right\}$.

Proposition 4.4.4. Let $\left(b, b^{\prime}\right)$ be a pair of 3-braids such that $w\left(b^{\wedge}\right)=w\left(b^{\prime \wedge}\right)$ and $V\left(b^{\wedge}\right)(s)=V\left(b^{\prime \wedge}\right)(s)$ for all $s \in C^{\bullet}$. Then, for $t \in C^{\bullet}, V^{(0,2)}\left(b^{\wedge}\right)(t)=V^{(2,0)}\left(b^{\prime \wedge}\right)$ (t) iff $\omega_{6,2}(b)(t)=\omega_{6,2}\left(b^{\prime}\right)(t)$.

Proof. Recall that $a_{n, i}(V)(t) \neq 0$ for $0 \leq i \leq[n / 2]$ by (4.1.11). From (4.1.10) and Theorem 1.4.10, it is enough to show that

$$
\omega_{6, i}(b)(t)=\omega_{6, i}\left(b^{\prime}\right)(t) \quad \text { for } \quad i=0,1,3 .
$$

We have $\omega_{6, i}(b)(t)=\omega_{6, i}\left(b^{\prime}\right)(t)$ for $i=1,3$ and $\chi_{3,0}(b)(t)=\chi_{3,0}\left(b^{\prime}\right)(t)$ since they are linear characters of $B_{3}$ and $w\left(b^{\wedge}\right)=v\left(b^{\prime \wedge}\right)$. The assumptions $w\left(b^{\wedge}\right)=v\left(b^{\prime \wedge}\right)$ 
and $V\left(b^{\wedge}\right)(s)=V\left(b^{\prime \wedge}\right)(s)$ for all $s \in C^{\bullet}$ yields $\chi_{3,1}(b)(s)=\chi_{3,1}\left(b^{\prime}\right)(s)$ since $V(\cdot)(s)=$ $a_{3,0}(V)(s) \chi_{3,0}(\cdot)(s)+a_{3,1}(V)(s) \chi_{3,1}(\cdot)(s)$ for 3-braids. But we know that $\omega_{6,1}$ $(b)(t)=(-1)^{w\left(b^{\wedge}\right)} \chi_{3,1}(b)\left(t^{2}\right)$, and so we have $\omega_{6,1}(b)(t)=\omega_{6,1}\left(b^{\prime}\right)(t)$. Hence (4.4.5) is proved.

From the above Proposition and Corollary 4.2.3, we have the following.

Corollary 4.4.6. Let $\left(b, b^{\prime}\right)$ be a pair of 3-braids such that $w\left(b^{\wedge}\right)=w\left(b^{\prime \wedge}\right)$ and $V\left(b^{\wedge}\right)(s)=V\left(b^{\wedge \wedge}\right)(s)$ for all $s \in C^{*}$. Then, for $t \in C^{*}, V^{(2)}\left(b^{\wedge}\right)(t)=V^{(2)}\left(b^{\prime \wedge}\right)(t)$ iff $\omega_{6,2}(b)(t)=\omega_{6,2}\left(b^{\prime}\right)(t)$.

Let $P=P(\cdot)(\alpha, q)$ denote the two-variable Jones polynomial with non-zero complex parameters $\alpha$ and $q$ where $q$ is not equal to any roots of unity. Then we have the following.

Corollary 4.4.7. Let $\left(b, b^{\prime}\right)$ be a pair of 3-braids such that $w\left(b^{\wedge}\right)=w\left(b^{\prime \wedge}\right)$, $V\left(b^{\wedge}\right)(s)=V\left(b^{\prime \wedge}\right)(s)$ and $V^{(2)}\left(b^{\wedge}\right)(s)=V^{(2)}\left(b^{\prime \wedge}\right)(s)$ for all $s \in C^{*}$. Then we have

(a) $P^{(2,(2))}\left(b^{\wedge}\right)(\alpha, q)=P^{(2,(2))}\left(b^{\prime \wedge}\right)(\alpha, q)$,

(b) $P^{(2,(11))}\left(b^{\wedge}\right)(\alpha, q)=P^{(2,(11))}\left(b^{\wedge}\right)(\alpha, q)$,

(c) $P^{(2)}\left(b^{\wedge}\right)(\alpha, q)=P^{(2)}\left(b^{\prime \wedge}\right)(\alpha, q)$.

Proof. The representations $\pi_{\lambda, \nu}(\lambda \in \Lambda(6), \nu=(2) \in \Lambda(2))$ of $B_{3}$ associated with $P^{(2)}$ are given in Table 3. With these representation matrices, an analogous argument of the proof of Proposition 4.4.4 shows the part (a). The part (a) and (3.6) implies the part (b). The part (c) follows from the parts (a), (b) and Theorem 1.5.1.

Proposition 4.4.8. Let $\left(b, b^{\prime}\right)$ be a pair of 3-braids whose closures are knot with $w\left(b^{\wedge}\right)=w\left(b^{\prime \wedge}\right)$ and $F\left(b^{\wedge}\right)(a, x)=F\left(b^{\prime \wedge}\right)(a, x)$ for all $a, x \in C^{\times}$, where $F$ denotes the Kauffman polynomial. Then, for $t \in C^{\bullet}, V^{(3,0)}\left(b^{\wedge}\right)(t)=V^{(3,0)}\left(b^{\prime \wedge}\right)(t)$ iff $\omega_{9,3}$ (b) $(t)=\omega_{9,3}\left(b^{\prime}\right)(t)$.

Proof. Because $a_{9,3}(V)(t) \neq 0$, it is enough to show that $\omega_{9, i}(b)(t)=\omega_{9, i}$ $\left(b^{\prime}\right)(t)$ for $i=0,1,2,4$. We have $V\left(b^{\wedge}\right)(s)=V\left(b^{\wedge}\right)(s)\left(s \in C^{\bullet}\right)$ from $F\left(b^{\wedge}\right)=F\left(b^{\prime \wedge}\right)$ [22]. Thus, as the proof of Proposition 4.4.4, we have $\omega_{9, i}(b)(t)=\omega_{9, i}\left(b^{\prime}\right)(t)$ for $i=0,1,4$. Let $\chi$ be the character of the representation $\rho$ of $B_{3}$ defined by the following.

$$
\rho\left(\sigma_{1}\right)=\left(\begin{array}{rrr}
a^{-1} & 0 & x \\
0 & 0 & -1 \\
0 & 1 & x
\end{array}\right), \quad \rho\left(\sigma_{2}\right)=\left(\begin{array}{ccr}
0 & -1 & 0 \\
1 & x & 0 \\
0 & a^{-1} x & a^{-1}
\end{array}\right) .
$$

From Theorem 12.2 of [26] we have $\chi(b)(a, x)=\chi\left(b^{\prime}\right)(a, x)$ if the values of $a$ and $x$ are generic. This yields $\chi(b)(a, x)=\chi\left(b^{\prime}\right)(a, x)$ for all $a, x \in C^{\times}$since $\chi(\beta)$ $(a, x)$ are Laurent polynomials of the parameters $a$ and $x$ for all $\beta \in B_{3}$. Let $\chi^{\prime}$ 
be the character obtained from $\chi$ by substituting $x=-t^{-3 / 2}+t^{3 / 2}$ and $a=t^{-7 / 2}$. Then $\omega_{9,2}(\cdot)(t)=t^{3} \chi^{\prime}(\cdot)(t)$ and so we have $\omega_{9,2}(b)(t)=\omega_{9,2}\left(b^{\prime}\right)(t)$. This proves the proposition.

From the above proposition and Corollary 4.2.3, we have the following.

Corollary 4.4.9. Let $\left(b, b^{\prime}\right)$ be a pair of 3-braids whose closures are knot with $w\left(b^{\wedge}\right)=w\left(b^{\prime \wedge}\right)$ and $F\left(b^{\wedge}\right)(a, x)=F\left(b^{\wedge}\right)(a, x)$ for all $a, x \in C^{\times}$, where $F$ denotes the Kauffman polynomial. Then, for $t \in C^{*}, V^{(3)}\left(b^{\wedge}\right)(t)=V^{(3)}\left(b^{\prime}\right)(t)$ iff $\omega_{\dot{9}, 3}(b)(t)=\omega_{9,3}$ $\left(b^{\prime}\right)(t)$.

Proposition 4.4.10. The invariant $V^{(3)}$ is independent from $V, V^{(2)}$, and the Kauffman polynomial $F$.

Proof. We give a pair of braids $\left(b, b^{\prime}\right)$ such that $V\left(b^{\wedge}\right)=V\left(b^{\wedge \wedge}\right), V^{(2)}\left(b^{\wedge}\right)=$ $V^{(2)}\left(b^{\prime \wedge}\right), F\left(b^{\wedge}\right)=F\left(b^{\prime \wedge}\right)$ but $V^{(3)}\left(b^{\wedge}\right) \neq V^{(3)}\left(b^{\prime \wedge}\right)$. Let

$$
b(m, n)=\sigma_{1}^{2} \sigma_{2}^{-2} \sigma_{1} \sigma_{2}^{2} \sigma_{1}^{-2} \sigma_{2}^{m} \sigma_{1}^{2} \sigma_{2}^{-2} \sigma_{1}^{-1} \sigma_{2}^{2} \sigma_{1}^{-2} \sigma_{2}^{n},
$$

for odd integers $m, n$. Then T. Kanenobu noted that the knots $b(m, n)^{\wedge}$ and $b(n, m)^{\wedge}$ have the same Kauffman polynomial. Therefore they have the same Jones polynomial [22] and the same 2-parallel version of the Jones polynomial [35]. But T. Kanenobu conjectured that $b(m, n)^{\wedge}$ and $b(n, m)^{\wedge}$ are not equivalent except a finite number of pairs $(m, n)$. From a calculation with a computer we have $\omega_{9,3}(b(3,1)) \neq \omega_{9,3}(b(1,3))$ by substituting 4 to the parameter $t$. Thus we have $V^{(3)}\left(b(1,3)^{\wedge}\right) \neq V^{(3)}\left(b(3,1)^{\wedge}\right)$ from Corollary 4.4.9.

From now on, we prepare some notations which is needed in the proof of Theorem 4.1.1. We use the notations in Section 4.1. Fix an integer $i$ with $0 \leq i \leq[3 r / 2]$. Let

$$
V_{3 r, i}=J_{3 r, i} e_{1} e_{3} \cdots e_{2 i-1} /\left(J_{3 r, i} e_{1} e_{3} \cdots e_{2 i-1} \cap J_{3 r, i+1}^{\cdot}\right) \text {. }
$$

From Proposition 4.1.9, $V_{3 r, i}$ is an irreducible left $J_{3 r}$-module and the left action of $J_{3 r}$ on $V_{3 r, i}$ are equivalent to $\rho_{r, i}$. By Proposition 4.1.6, there is a subset $G_{3 r, i}$ of $E_{3 r}$ such that $\left\{x \bmod J_{3 r, i+1}^{*} \mid x \in G_{3 r, i}\right\}$ is a basis of $V_{3 r, i}$. More precisely, $G_{3 r, i}=E_{r, i} \cap J_{3 r} e_{1} e_{3} \cdots e_{2 i-1}$. Let

$$
\begin{aligned}
G_{3 r, i, 1}=\left\{x \in G_{3 r, i} \mid\right. & \text { there is } j \neq 0(\bmod r) \text { such that the } j \text {-th point at the } \\
& \text { top of } \eta(x) \text { is connected to the }(j+1) \text {-th point at the } \\
& \text { top of } \eta(x)\},
\end{aligned}
$$

and $V_{3 r, i, 1}$ the subspace of $V_{3 r, i}$ spanned by the image of $G_{3 r, i, 1}$. For $0 \leq j_{1}, j_{2}, j_{3} \leq$ $[r / 2]$, let $V_{3 r, i, j_{1}, j_{2}, j_{3}}$ be the $\rho_{i, j_{1}} \otimes \rho_{r, j_{2}} \otimes \rho_{r, j_{3}}$-isotypic subspace with respect to $J_{r}^{\otimes 3}$ naturally embedded in $J_{3 r}$. Let $I=\{k \mid 1 \leq k \leq 3 r-1, k \neq 0 \bmod r\}$ and $J_{I}$ the 
subalgebra of $J_{3 r}$ generated by $e_{k}(k \in I)$. Let $\lambda_{0}$ denote the one-dimensional representation of $J_{I}$ defined by $\lambda_{0}\left(e_{k}\right)=0(k \in I)$. For a $J_{I}$-module $U$, let $U_{+}=\sum_{k \in I} e_{k} U$. Then $U_{+}$is a $J_{I}$-submodule of $U$. Let $U_{0}$ denote the $\lambda_{0}$-isotypic subspace of $U$. Since $-\left(t^{1 / 2}+t^{-1 / 2}\right)^{-1} e_{k}(k \in I)$ is a projection and $U_{0}=$ $\bigcap_{k \in I}\left(1+\left(t^{1 / 2}+t^{-1 / 2}\right)^{-1} e_{k}\right) U$, we have the following.

Proposition 4.4.11. The composition of natural homomorphisms $U_{0} \rightarrow U \rightarrow$ $U / U_{+}$is a $J_{I}$-module isomorphism.

From Proposition 4.1.8, we have $V_{3 r, i, 1}=\sum_{k \in I} e_{k} V_{3 r, i}$. Since $J_{I}$ is isomorphic to $J_{r}^{\otimes 3}$, we get the following.

Corollary 4.4.12. The $J_{r}^{\otimes 3}$-submodule $V_{3, i, 0,0,0}$ of $V_{r, i}$ is isomorphic to $V_{3 r, i}$ $V_{3 r, i, 1}$ as a $J_{r}^{\otimes 3}$-module.

Let $V_{3 r, i, 0}=V_{3 r, i} / V_{3 r, i, 1}$. Then the above corollary implies that the representation $\pi_{3 r, i}$ of $B_{3}$ in Theorem 4.4.1 is equivalent to the action of $B_{3}$ on $V_{3 r, i, 0}$ induced by restricting the representation $\rho_{3 r, i} \circ \phi_{3}^{(r)}$. We give a basis of $V_{3 r, i, 0}$ and representation matrices of $\pi_{3 r, i}$ with respect to the above basis. For $0 \leq k, m<j$, let

$$
\begin{aligned}
& e^{(d)}(j-k, j+k, j-m, j+m)= \\
& \qquad\left\{\begin{array}{l}
e(j-k, j+k) e(j-k-1, j+k+1) \cdots e(j-m, j+m) \text { if } m \geq k, \\
e(j-k, j+k) e(j-k+1, j+k-1) \cdots e(j-m, j+m) \text { if } m<k,
\end{array}\right.
\end{aligned}
$$

and

$$
e^{(h)}(j-k, j+m, j-m, j+k)=e(j-k, j+m) e(j-k-1, j+m-1) \cdots e(j-m, j+k) .
$$

Let $H_{r, i}=\left\{h_{r, i, j} \mid 0 \leq j \leq i\right.$ if $i \leq r$ and $i-r \leq j \leq 2 r-i$ if $\left.r<i\right\}$, where the elements $h_{r, i, j}$ are given as follows. In the case of $0 \leq i \leq r$, let

$$
\begin{aligned}
& h_{r, i, j}{ }^{(1)}=e^{(d)}(r, r, r-j+1, r+j-1), \\
& h_{r, i, j}{ }^{(2)}=e^{(d)}(2 r, 2 r, 2 r-i+j+1,2 r+i-j-1), \\
& h_{r, i, j}{ }^{(3)}=e^{(h)}(2 r-i+j, 2 r+i-j-2, r+j+1, r-j+2 i-1), \\
& h_{r, i, j}{ }^{(4)}=e^{(h)}(r+j, r-j+2 i-2,1,2 i-2 j-1), \\
& h_{r, i, j}=h_{r, i, j}{ }^{(1)} h_{r, i, j}{ }^{(2)} h_{r, i, j}{ }^{(3)} h_{r, i, j}{ }^{(4)},
\end{aligned}
$$

for $0 \leq j \leq i$. In the case of $r<i \leq[3 r / 2]$, let

$$
\begin{aligned}
& h_{r, i, j}{ }^{(1)}=e^{(d)}(r, r, r-j+1, r+j-1), \\
& h_{r, i, j}{ }^{(2)}=e^{(d)}(2 r, 2 r, r+j+1,3 r-j-1), \\
& h_{r, i, j}{ }^{(3)}=e^{(d)}(r-j, 3 r-j, 2 r-i-j+1,2 r+i-j-1), \\
& h_{r, i, j}{ }^{(4)}=e^{(h)}(2 r-i-j, 2 r+i-j-2,1,2 i-1),
\end{aligned}
$$




$$
h_{r, i, j}=h_{r, i, j}{ }^{(1)} h_{r, i, j}{ }^{(2)} h_{r, i, j}{ }^{(3)} h_{r, i, j}{ }^{(1)} \text {. }
$$

for $i-r \leq j \leq 2 r-i$. The rectangular diagrams of $\eta\left(h_{r, i, j}\right) \in D_{3 r}$ are given as in Figure 15.

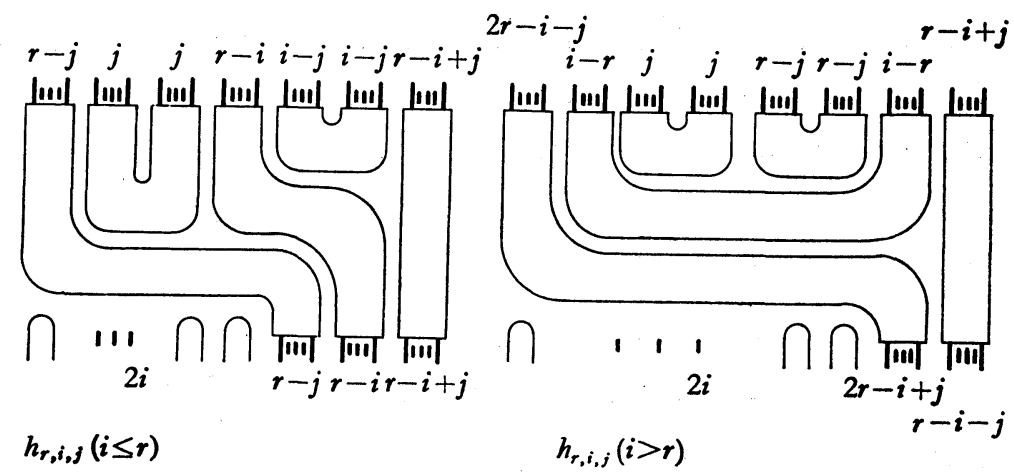

Figure 15

Let $W_{3 r, i}$ denote the inverse image of $V_{3 r, i, 1}$ with respect to the canonical projection

$$
J_{3 r} e_{1} e_{3} \cdots e_{2 i-1} \rightarrow J_{3 r} e_{1} e_{3} \cdots e_{2 i-1} /\left(J_{3 r, i+1} \cap J_{3 r} e_{1} e_{3} \cdots e_{2 i-1}\right) \text {. }
$$

Proposition 4.4.13. The set $H_{r, i}=\left\{h \bmod W_{3 r, i} \mid h \in H_{r, i}\right\}$ is a basis of $V_{3 r, i, 0}$

Proof. For $h \in H_{r, i}$, we know that $h \in E_{3 r, i}, h \in J_{3 r} e_{1} e_{3} \cdots e_{2 i-1}, h \notin E_{3 r, i+1}$ and $h \notin E_{3 r, i, 1}$. Hence $H_{r, i}$ is linearly independent since $W_{3 r, i}$ is a subspace of $J_{r, i}^{\bullet}$ spanned by $\left(E_{r, i+1} \cap J_{3 r} e_{1} e_{3} \cdots e_{2 i-1}\right) \cup E_{r, i, 1}$. Since the dimension of the representation $\rho_{r, 0}^{\otimes 3}$ of $J_{r}^{\otimes 3}$ is equal to one, the dimension of the $\rho_{r, 0}^{\otimes 3}$-isotypic subspace of $V_{3 r, i}$ with respec to $J_{r}^{\otimes 3}$ is equal to the multiplicity of $\rho_{r, 0}^{\otimes 3}$ in $\left.\rho_{3 r, i}\right|_{J_{r}^{\otimes 3}} ^{\otimes}$. From Remarks 4.1.12 and 3.8, we may use the Littlewood-Richardson rule (Remark 3.8) for computing $\operatorname{dim}_{C} V_{3 r i, 0}$. The result is

$$
\operatorname{dim}_{C} V_{3 r, i, 0}=i+1 \text { if } 0 \leq i \leq r \text { and } 2 r-i+1 \text { if } r<i \leq[3 r / 2] \text {, }
$$

which is equal to $\# H_{r, i}$. This shows that $H_{3 r, i}$ is a basis of $V_{3 r, i, 0}$.

Let $p_{3 r}: B_{3 r} \rightarrow J_{3 r}$ be the algebra homomorphism introduced in Section 4.1. We use the following formulas in the proof of Theorem 4.4.1, which can be proved immediately from the definitions of $J_{3 r}, p_{3 r}$ and $W_{3 r, i}$. For $v \in J_{r} e_{1} e_{3} \cdots$ $e_{2 i-1}$ and $1 \leq k \leq 3 r-1$ with $k \neq 0(\bmod r)$, we have

$$
p_{3 r}\left(\sigma_{i}\right) v=-t^{1 / 2} v \quad\left(\bmod W_{3 r, i}\right) .
$$

For $1 \leq k \leq 3 r-1$, we have 


$$
p_{3 r}\left(\sigma_{i}\right) e_{i}=e_{i} p_{3 r}\left(\sigma_{i}\right)=t^{3 / 2} e_{i} .
$$

For $1 \leq k \leq 3 r-2$, we have

$$
\begin{aligned}
& p_{3 r}\left(\sigma_{1}\right) e_{i+1} e_{i}=t^{3 / 2} p_{3 r}\left(\sigma_{i+1}\right)^{-1} e_{i}, \quad p_{3 r}\left(\sigma_{i+1}\right) e_{i} e_{i+1}=t^{3 / 2} p_{3 r}\left(\sigma_{i}\right)^{-1} e_{i+1}, \\
& p_{3 r}\left(\sigma_{i}\right)^{-1} e_{i+1} e_{i}=t^{-3 / 2} p_{3 r}\left(\sigma_{i+1}\right) e_{i}, \quad p_{3 r}\left(\sigma_{i+1}\right)^{-1} e_{i} e_{i+1}=t^{-3 / 2} p_{3 r}\left(\sigma_{i}\right) e_{i+1}, \\
& p_{3 r}\left(\sigma_{i}\right) e_{i+1} p_{3 r}\left(\sigma_{i}\right)^{-1}=p_{3 r}\left(\sigma_{i+1}\right)^{-1} e_{i} p_{3 r}\left(\sigma_{i+1}\right), \\
& p_{3 r}\left(\sigma_{i}\right)^{-1} e_{i+1} p_{3 r}\left(\sigma_{i}\right)=p_{3 r}\left(\sigma_{i+1}\right) e_{i} p_{3 r}\left(\sigma_{i-1}\right)^{-1}
\end{aligned}
$$

We also need following formulas, which can be proved by using above formulas. For $p, q \in \boldsymbol{N}$ with $p \leq q$, let

$$
\begin{aligned}
& \sigma(p, q)=\sigma_{p} \sigma_{p+1} \cdots \sigma_{q}, \quad \sigma^{-}(p, q)=\sigma_{p}^{-1} \sigma_{p+1}^{-1} \cdots \sigma_{q}^{-1}, \\
& \sigma^{\prime}(q, p)=\sigma_{q} \sigma_{q-1} \cdots \sigma_{p} \text { and } \sigma^{-}(q, p)=\sigma_{q}^{-1} \sigma_{q-1}^{-1} \cdots \sigma_{p}^{-1} .
\end{aligned}
$$

For $0 \leq k \leq m<j$, let

$$
\sigma(j \pm k, j+m, j-m, j \mp k)=\sigma(j \pm k, j+m) \sigma(j \pm k-1, j+m-1) \cdots \sigma(j-m, j \mp k) .
$$

and

$$
\begin{gathered}
\sigma^{-}(j \pm k, j+m, j-m, j \pm k)=\sigma^{-}(j \pm k, j+m) \\
\sigma^{-}(j \pm k-1, j+m-1) \cdots \sigma^{-}(j-m, j \pm k) .
\end{gathered}
$$

For $j, k, m, n \in N$ with $j>m-k+n$, we have

$$
\begin{aligned}
& \sigma(j-k+m, j+m+n-1, j-k+1, j+n) \sigma(j-k, j-k-n+1, j-1, j-n) \\
& e^{(d)}(j, j, j-n+1, j+n-1) \\
& =\sigma^{-}(j-k, j-k+1, j+k+m-1, j-k-n+m) \\
& \quad \sigma(j-k+m, j+m-1, j-k-n+1, j-n) \\
& \quad e^{(d)}(j+m, j+m, j+m-n+1, j+m+n-1) \\
& \quad e^{(h)}(j+m-n, j+m+n-2, j-n+1, j+n-1)
\end{aligned}
$$

For $j, k \in N$, we have

$$
\begin{aligned}
& \sigma_{i}^{ \pm 1} e(i+2, i+2 k) e(i+1, i+2 k-1) e(i, i+2 k-2) \\
& \quad=e(i+2, i+2 k) e(i+1, i+2 k-1) e(i, i+2 k-2) \sigma_{i+2 k}^{ \pm 1} .
\end{aligned}
$$

Formula (4.4.17) with $m=1,(4.4 .15),(4.4 .16)$ and (4.4.18) implies that

$$
\begin{gathered}
\sigma(j, j+k-1) \sigma^{\prime}(j-1, j-k+1) e^{(d)}(j, j, j-k+1, j+k-1) \\
=t^{3 / 2} t^{3(k-1)} \sigma^{\prime}(j-1, j-k+1) \sigma^{\prime}(j-1, j-k+1)^{-1} \\
e^{(d)}(j, j, j-k+1, j+k-1) .
\end{gathered}
$$

An induction on $m$ and (4.4.19) shows that 
(4.4.20) $\sigma(j, j-m+1, j+m-1, j) e^{(d)}(j, j, j-m+1, j+m-1)$

$=\left(t^{3 / 2}\right)^{m 2} \sigma^{\prime-}(j-1, j-m+1) \sigma^{\prime}(j-1, j-m+1)^{-1}$

$\sigma^{\prime-}(j-2, j-m+1) \sigma^{\prime}(j-2, j-m+1)^{-1}$

$\cdots \sigma^{\prime-}(j-m, j-m) \sigma^{\prime}(j-m, j-m)^{-1} e^{(d)}(j, j, j-m+1, j+m-1)$.

Proof of Theorem 4.4.1. We calculate the representation matrices of $\pi_{3 r, i}$ $\left(\sigma_{j}\right)(j=1,2)$ with respect to the basis $H_{3 r, i}$. This can be done by writing down $p_{3 r}\left(\phi_{3}^{(r)}\left(\sigma_{j}\right)\right) h_{r, i, k} \bmod W_{3 r, i}(j=1,2)$ as $\boldsymbol{C}$-linear combinations of elements of $H_{3 r, i} \cdot$. Steps $1-7$ can be proved by using formulas (4.4.14)-(4.4.20).

STEP 1. $p_{3 r}\left(\phi_{3}^{(r)}\left(\sigma_{j}\right)\right) h_{r, i k}$

$$
\equiv\left(-t^{1 / 2}\right)^{r(r-1)} \sigma(r, 2 r-1,1, r) h_{r, i, k}\left(\bmod W_{s r, i}\right) \text {. }
$$

STEP 2. $\sigma(r, 2 r-1,1, r) h_{r, i, k}$

$$
\begin{aligned}
\equiv & \left(t^{3 / 2}\right)^{k^{2}} \sigma(r, 2 r-1, k+1, r+k) \sigma(k, r-1,1, r-k) \\
& \sigma^{\prime-}(r-1, r-k+1) \sigma^{\prime}(r-1, r-k+1)^{-1} \sigma^{\prime-}(r-2, r-k+2) \\
& \sigma^{\prime}(r-2, r-k+2)^{-1} \cdots \sigma^{\prime-}(r-k+1, r-k+1) \\
& \sigma^{\prime}(r-k+1, r-k+1)^{-1} h_{r, i, k}\left(\bmod W_{3 r, i}\right) .
\end{aligned}
$$

STEP 3. $\sigma(r, 2 r-1, k+1, r+k) \sigma(k, r-1,1, r-k)$

$$
\begin{aligned}
& \sigma^{\prime-}(r-1, r-k+1) \sigma^{\prime}(r-1, r-k+1)^{-1} \sigma^{\prime-}(r-2, r-k+2) \\
& \sigma^{\prime}(r-2, r-k+2)^{-1} \cdots \sigma^{\prime-}(r-k+1, r-k+1) \\
& \sigma^{\prime}(r-k+1, r-k+1)^{-1} h_{r, i, k} \\
& \quad \equiv\left(-t^{-1 / 2}\right)^{k(k-1)} \sigma(r, 2 r-1, k+1, r+k) \sigma(k, r-1,1, r-k) h_{r, i, k} \\
& \quad\left(\bmod W_{3 r, i}\right) .
\end{aligned}
$$

STEP 4. $\sigma(r, 2 r-1, k+1, r+k) \sigma(k, r-1,1, r-k) h_{r, i, k}{ }^{(2)}$

$$
\begin{aligned}
= & t^{3 k(r-k) / 2} \sigma^{-}(k, r-1,1, r-k) \sigma(r, 2 r-k-1, r-k+1,2 r-2 k) \\
& \sigma(r-k, 2 r-2 k-1,1, r-k) \\
& e^{(d)}(2 e r-k, 2 r-k, 2 r-2 k+1,2 r-1) \\
& e^{(h)}(2 r-2 k, 2 r-2, r-k+1, r+k-1) .
\end{aligned}
$$

STEP 5. $\sigma^{-}(k, r-1,1, r-k) \sigma(r, 2 r-k-1,1, r-k)$

$$
\begin{aligned}
& e^{(d)}(2 r-k, 2 r-k, 2 r-2 k+1,2 r-1) \\
& e^{(h)}(2 r-2 k, 2 r-2, r-k+1, r+k-1) \\
& e^{(d)}(2 r, 2 r, 2 r-i+k+1,2 r+i-k-1) \\
& e^{(h)}(2 r-i+k, 2 r+i-k-2, r+k+1, r-k+2 i-1) \\
& e^{(h)}(r-k, r-k+2 i-2,1,2 i-1) \\
& \quad \equiv\left(-t^{-1 / 2}\right)^{k(r-k)} \sigma(r-k, 2 r-2 k-1,1, r-k)
\end{aligned}
$$




$$
\begin{aligned}
& e^{(d)}(2 r-k, 2 r-k, 2 r-i-k+1,2 r+i-k-1) \\
& e^{(k)}(2 r-i-k, 2 r+i-k-2,1,2 i-1)\left(\bmod W_{\mathrm{sr}, i}\right) .
\end{aligned}
$$

STEP 6. $\sigma(r-k, 2 r-2 k-1,1, r-k)$

$e^{(d)}(2 r-k, 2 r-k, 2 r-i-k+1,2 r+i-k-1)$

$e^{(h)}(2 r-i-k, 2 r+i-k-2,1,2 i-1)$

$$
\begin{aligned}
\equiv & t^{3 i(r-k) / 2}\left(-t^{1 / 2}\right)^{(r-i)(r-k)-k(r-k)} \sigma^{-}(2 r, r+k+1,2 r+i-k-1, r+i) \\
& e^{(d)}(r, r, r-i+1, r+i-1) e^{(h)}(r-i, r+i-2,1,2 i-1) \\
& \left(\bmod W_{3 r, i}\right) .
\end{aligned}
$$

STEP 7. For non-negative integers $i, k$ and $m$ such that $k<i$ and $m<i-k$, we have

$$
\begin{aligned}
& e^{(d)}(2 r, 2 r, 2 r+m-2,2 r-m+2) \\
& e^{(h)}(2 r-m+1,2 r+m-1, r+k+1, r+k+2 m-1) \\
& \sigma^{-}(2 r+m, r+k+2 m+1,2 r+i-k-1, r+i+m) \\
& e^{(d)}(r, r, r-k+1, r+k-1) \\
& e^{(d)}(r-k, r+k+2 m, r-i+m+1, r+i+m-1) \\
& e^{(h)}(r-i+m, r+i+m-2,1,2 i-1) \\
& \equiv\left(-t^{-1 / 2}\right)^{(r-2 k-2 m+i-1)} e^{(d)}(2 r, 2 r, 2 r+m-2,2 r-m+2) \\
& \quad e^{(h)}(2 r-m+1,2 r+m-1, r+k+2, r+k+2 m) \\
& \quad \sigma^{-}(2 r+m, r+k+2 m+2,2 r+i-k-2, r+i+m) \\
& \quad e^{(d)}(r, r, r-k, r+k) \\
& \quad e^{(d)}(r-k-1, r+k+2 m+1, r-i+m+1, r+i+m-1) \\
& \quad e^{(h)}(r-i+m, r+i+m-2,1,2 i-1) \\
& \quad+\left(-t^{-1}\right)^{(r-2 k-2 m+i-1)} e^{(d)}(2 r, 2 r, 2 r+m-1,2 r-m+1) \\
& \quad e^{(h)}(2 r-m, 2 r+m, r+k+1, r+k+2 m+1) \\
& \quad \sigma^{-}(2 r+m+1, r+k+2 m+3,2 r+i-k-1, r+i+m+1) \\
& \quad e^{(d)}(r, r, r-k+1, r+k-1) \\
& \quad e^{(d)}(r-k, r+k+2 m+2, r-i+m+2, r+i+m) \\
& e^{(h)}(r-i+m+1, r+i+m-1,1,2 i-1)\left(\bmod W_{3 r, i}\right) .
\end{aligned}
$$

Step 7 implies the following.

STEP 8. $e^{(d)}(2 r, 2 r, 2 r+m-2,2 r-m+2)$

$$
\begin{aligned}
& e^{(k)}(2 r-m+1,2 t+m-1, r+k+1, r+k+2 m-1) \\
& \sigma^{-}(2 r+m, r+k+2 m+1,2 r+i-k-1, r+i+m) \\
& e^{(d)}(r, r, r-k+1, r+k-1)
\end{aligned}
$$




$$
\begin{aligned}
& e^{(d)}(r-k, r+k+2 m, r-i+m+1, r+i+m-1) \\
& e^{(h)}(r-i+m, r+i+m-2,1,2 i-1) \\
& \quad \equiv \sum_{j=k}^{i} \beta_{k}, h_{r, i, j},
\end{aligned}
$$

where $\beta_{k, m, j}(0 \leq m \leq i-k)$, satisfy the following relations.

$$
\begin{aligned}
& \beta_{k, i-k, k}=1(0 \leq k \leq i), \quad \beta_{k, i-k, j}=0(0 \leq j \leq i, j \neq k), \\
& \beta_{k, m, j}=\left(-t^{-1 / 2}\right)^{(r-2 k-2 m+i-1)} \beta_{k+1, m, j}+\left(-t^{-1}\right)^{(r-2 k-2 m+i-1)} \beta_{k, m+1, j} \\
& (0 \leq m<i-k) .
\end{aligned}
$$

STEP 9. For $0 \leq k \leq j$ and $0 \leq m \leq i-j$, we have

$$
\begin{aligned}
& \beta_{k, m, j} \\
& \quad=(-1)^{(r-i+1)(i-k-m)}\left(t^{-1 / 2}\right)^{(r-i+j-k)(j-k)+2(r+j-2 k-m)(i-j-m)} g_{1-k-m, j-k}(t) .
\end{aligned}
$$

Especially, we have

$$
\beta_{k, 0, j}=(-1)^{(r-i+1)(i-k)}\left(t^{-1 / 2}\right)^{(r-i+j-k)(j-k)+2(r+j-2 k)(i-j)} g_{i-k, j-k}(t) .
$$

Proof. Since

$$
(-1)^{(r-i+1)(i-k-m)}\left(t^{-1 / 2}\right)^{(r-i+j-k)(j-k)+2(r+j-2 k-m)(i-j-m)} g_{i-k-m, j-k}(t)
$$

satisfy the relation (4.4.21), these are identical to $\beta_{k, m, j}$.

STEP 10. Combining the results of Steps 1-9, we get the statement of Theorem 4.4.1 for the case $0 \leq i \leq r$. An analogous argument proves the statement of the theorem for the case $r<i \leq[3 r / 2]$.

5. The parallel version of the Kauffman polynomial. The associated algebras $C_{1}, C_{2}, \cdots$ of the Kauffman polynomial $F$ (Definition 1.1.10) are obtained in [2] and [26]. The invariant $F$ is known to be of trace type if the values of the parameters $a$ and $x$ are generic. Therefore we can apply our theory in Section 1 to $F$ with generic parameters. We give a formula for the Kauffman polynomials of satellite links (Theorem 5.3.1).

5.1. The associated algebra $C_{n}$. Let $a, \beta \in C \backslash\{0\}$ such that $\beta$ is not equal to any root of unity. Let $x=\beta-1 / \beta$. The associated algebra $C_{n}$ of the Kauffman polynomial is defined as a $\boldsymbol{C}$-algebra with 1 by the following.

$$
\begin{aligned}
& C_{n}= \\
& \quad\left\langle\tau_{i}, \tau_{i}^{-1}, \varepsilon_{i}(1 \leq i \leq n-1)\right| \tau_{i} \tau_{i+1} \tau_{i}=\tau_{i+1} \tau_{i} \tau_{i+1}, \\
& \quad \varepsilon_{i} \varepsilon_{i+1} \varepsilon_{i}=\varepsilon_{i}, \varepsilon_{i+1} \varepsilon_{i} \varepsilon_{i+1}=\varepsilon_{i+1} \\
& \quad \tau_{i}^{ \pm 1} \varepsilon_{i+1} \varepsilon_{i}=\tau_{i+1}^{\mp 1} \varepsilon_{i}, \tau_{i+1}^{ \pm 1} \varepsilon_{i} \varepsilon_{i+1}=\tau_{i}^{\mp 1} \varepsilon_{i+1}
\end{aligned}
$$




$$
\begin{aligned}
& \varepsilon_{i} \varepsilon_{i+1} \tau_{i}^{\ddagger 1}=\varepsilon_{i} \tau_{i+1}^{\mp 1}, \varepsilon_{i+1} \varepsilon_{i} \tau_{i+1}^{+1}=\varepsilon_{i+1} \tau_{i}^{\mp 1} \quad(1 \leq i \leq n-2), \\
& \tau_{i} \tau_{j}=\tau_{j} \tau_{i}, \varepsilon_{i} \tau_{j}=\tau_{j} \varepsilon_{i}, \varepsilon_{i} \varepsilon_{j}=\varepsilon_{j} \varepsilon_{i} \quad(1 \leq i \leq j-1 \leq n-2), \\
& \tau_{i}-\tau_{j}^{-1}=x\left(1-\varepsilon_{i}\right), \tau_{i} \varepsilon_{i}=\varepsilon_{i} \tau_{i}=a^{-1} \varepsilon_{i}, \tau_{i} \tau_{i}^{-1}=\tau_{i}^{-1} \tau_{i}=1 \\
& \quad(1 \leq i \leq n-1)\rangle .
\end{aligned}
$$

This definition is equivalent to the one in [2]. Let $C_{n, i}=C_{n} e_{1} e_{3} \cdots e_{2 i-1} C_{n}$. Then $C_{n, i}$ is a two sided ideal and satisfies $C_{n}=C_{n, 0} \supset C_{n, 1} \supset \cdots \supset C_{n,[n / 2]}$. Let $D_{n, i}=C_{n, i} / C_{n, i+1}(0 \leq i \leq[n / 2]-1)$ and $D_{n,[n / 2]}=C_{n,[n / 2]}$. Note that

$$
\left.D_{n, 0} \simeq H_{n}\left((-1)^{-1 / 2} \beta\right) \text { (the Iwahori's Hecke algebra of type } A_{n-1}\right) \text {. }
$$

Let $c_{n, i}=\left(\begin{array}{c}n \\ 2\end{array}\right)\left(\begin{array}{c}n-2 \\ 2\end{array}\right) \cdots\left(\begin{array}{c}n-2 i \\ 2\end{array}\right) /(i !)$. For $\mu \in \Lambda(n)$, let $d(\mu)$ denote the degree of the character $\chi_{\mu}$ of $H_{n}$ parametrized by the partition $\mu$. Then we have ([2], Theorem 3.7)

$$
D_{n, i} \equiv \bigoplus_{\mu \in \Lambda(n-2 i)} M_{d(\mu)_{c_{n, i}}}(C) \quad(0 \leq i \leq[n / 2]) .
$$

Hence the irreducible representations of $D_{n, i}$ are parametrized by $\Lambda(n-2 i)$ and so

$$
C_{n}{ }^{\wedge}=\{(i, \mu) \mid 0 \leq i \leq[n / 2], \mu \in \Lambda(n-2 i)\} .
$$

Let $\rho_{i, \mu}$ denote the corresponding irreducible representation of $(i, \mu)$ and $\chi_{i, \mu}$ its character. Let $p_{n}^{\prime}: C G_{n} \rightarrow C_{n}$ be the homomorphism defined by $p_{n}^{\prime}\left(\sigma_{i}\right)=\tau_{i}, p_{n}^{\prime}\left(\sigma_{i}^{-1}\right)$ $=\tau_{i}^{-1}, p_{n}^{\prime}\left(f_{i}\right)=\varepsilon_{i}$, and $p_{n}: C B_{n} \rightarrow C_{n}$ the homomorphism defined by $p_{n}\left(\sigma_{i}\right)=a \tau_{i}$ and $p_{n}\left(\sigma_{i}^{-1}\right)=a^{-1} \tau_{i}^{-1}$ for $1 \leq i \leq n-1$. Then the main result of [2] can be reformulated as follows.

Theorem 5.1.5. For an $n$-braid $b$, there are $a_{i, \mu}(F) \in C(0 \leq i \leq[n / 2], \mu \in \Lambda$ $(n-2 i))$ such that

$$
F\left((b, n)^{\wedge}\right)=\sum_{(i, \mu) \in \sigma_{n} \wedge} a_{i, \mu}(F) \chi_{i, \mu}\left(p_{n}(b)\right) .
$$

The coefficients $a_{i, \mu}(F)$ are given explicitly in [28].

5.2. Relations among the invariants associated with the decomposition of $F^{(r)}$. Let $F^{(r, i, \mu))}$ denote the invariant associated with the decomposition of the $r$-parallel version $F^{(r)}$ of the Kauffman polynomial $F$ parametrized by $(i, \mu) \in C_{n}{ }^{\wedge}(5.1 .4)$ as in Section 1.3. In this section we show that some of the above invariants are the same one. In the following we use the conventions that $F^{(0)}(K)=F^{(0,(0, \phi))}(K)=\left(-1+\left(a+a^{-1}\right) / x\right)^{-1}$ for any link diagram $K$.

Theorem 5.2.1. Let $K$ be a knot. Then we have 


$$
F^{(r,(i, \mu))}(K)=F^{(r-2 k,(i-k, \mu))}(K) \text { for } 0 \leq r, 0 \leq i \leq[r / 2], 0 \leq k \leq i .
$$

Corollary 5.2.2. Let $K$ be a knot. Then we have

$$
F^{(r)}(K)=\sum_{i=0}^{[r / 2]} \sum_{\mu \in \Lambda(r-2 i)} \chi_{i, \mu}(1) F^{(r-2 i,(0, \mu))}(K) .
$$

The above results may be generalized to the case of marked links.

We may prove Theorem 5.2 .1 by an anologous argument used in the proof of Theorem 4.2.1. We use the $D$-polynomial instead of the bracket polynomial. In this case, the following lemma takes the role of Lemma 4.2.7 in the proof of Theorem 4.2.1.

Lemma 5.2.3. Let $e(j)=e_{1} e_{3} \cdots e_{2 j-1} \in C_{r}(1 \leq j \leq[r / 2])$. For the character $\chi_{i, \mu}$ of the representation $\rho_{i, \mu}$ of $C_{r}$, we have
(a) $\chi_{i, \mu}(e(j))=0$
if $i<j$,
(b) $\chi_{i, \mu}(e(j))=\left(1-\left(a-a^{-1}\right) / x\right)^{j} \chi_{i-j, \mu}(1)$ if $1 \leq j \leq i$,

where $\chi_{i-j, \mu} \in C_{r-2 j} \hat{\text { and }}$ we use the convention that $\chi_{0, \phi}(1)=1$.

To prove this lemma, we use the folloaing formula in [2]. For $\mu=\left(\mu_{1}, \mu_{2}\right.$, $\cdots) \in \Lambda(n)$ and $\mu^{\prime}=\left(\mu_{1}^{\prime}, \mu_{2}^{\prime}, \cdots\right) \in \Lambda(n-1)$, we denote $\mu^{\prime}<\mu$ iff $\mu_{i}^{\prime} \leq \mu_{i}$ for all $i \in N$. For $y \in C_{r-1}, 0 \leq i \leq[r / 2]$ and $\mu \in \Lambda(r-2 i)$, we have

$$
\chi_{i, \mu}(y)=\sum_{\substack{\mu^{\prime} \in \Lambda(r-2 i-1), \mu^{\prime}<\mu}} \chi_{i, \mu^{\prime}}((y))+\left(\sum_{\substack{\mu^{\prime \prime} \in \Lambda(r-2 i+1) \\ \mu<\mu^{\prime \prime}}} \chi_{i-1, \mu^{\prime \prime}}(y)\right) .
$$

The details of proofs of Theorem 5.2.1 and Lemma 5.2.3 are omitted.

5.3. The Kauffman polynomial of satellite links. In the case of the Kauffman polynomial, we have a formula for srtellite links as in the case of the one-variable Jones polynomial (discussed in Section 4.3).

Theorem 5.3.1. Let $K$ be a knot, $L$ a link diagram in the annulus, and $(g, r)$ an element of $G$ whose closure is regular isotopic to $|L| . \quad$ Let $F_{L}(K)=F\left(K_{L}\right)$, where $K_{L}$ is the satellite link of $K$ with respect to $L$. Then we have

$$
F_{L}(K)=a^{-w(L)}\left(\sum_{(i, \mu) \in \sigma_{\hat{r}}} \chi_{i, \mu}\left(p_{r}^{\prime}(g)\right) F^{(i, \mu)}(K)\right) .
$$

The proof is similar to that of Theorem 4.3.2 and we omit it. This theorem may be generalized to the case of marked links.

EXAMPLE 5.3.2 (doubled knot [33]). Let $L_{0}$ be the link diagram in the annulus as in Figure 14. Then as in Example 4.3.3, $\left|L_{0}\right|$ is regular isotopic to $\left(g_{0}, 4\right)^{\sim}$, where $g_{0}=f_{1} f_{2} f_{3} \sigma_{2}^{2}$. For a knot $K$, we have $F_{L_{0}}(K)=-a^{-2} x\left(\beta^{-1}+a^{-1}\right)$ $F^{(2,(0,(2)))}(K)+a^{-2} x\left(\beta-a^{-1}\right) F^{(2,(0,(11)))}(K)+a^{-2}\left(x^{3}\left(a-a^{-1}-x\right)^{-1}+x^{2}+1\right)$ by calculating $\chi_{j, \mu}\left(g_{0}\right)$ for $(i, \mu) \in C_{4} \hat{~}$. 
6. Mutation and the r-parallel version of a link invariant. Let $K$ and $K^{\prime}$ be two distinct mutant links (Definition 6.2.3). We are interested in comparing the r-parallel versions $X^{(r)}(K)$ and $X^{(r)}\left(K^{\prime}\right)$ of a link nivariant $X$. In the case of the (two-variable) Jones polynomial $V, P$ and the Kauffman polynomial $F$, it is already known [23], [25] that none of $V^{(r)}, P^{(2)}$, and $F^{(2)}$ can distinguish two mutant knots. But it is announced [25] that the Kinoshita-Terasaka knot and the Conway's 11-corssing knot, which are mutant, have distinct $P^{(3)}$. We can show that there are four mutant knots having distinct $P^{(3)}$.

\subsection{Tangles.}

Definition 6.1.1. Let $n$ be a positive integer. An oriented rectangular diagram $T$ which consists of oriented curves is called a $n$-tangle if $T$ contains some or no closed curves, and $n$ non-closed curves starting at the top of the rectangle and terminating at the bottom of it (Figure 16). Two $n$-tangles $T$ and $S$
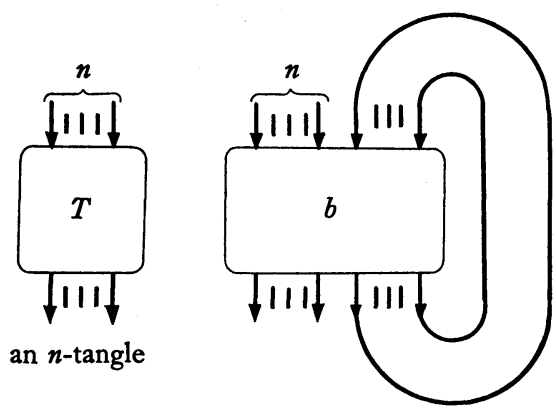

the $n$-closure of a braid $b$

Figure 16

are called isotopic if there is a sequence of Reidemeister moves from $T$ to $S$. We denote $R_{n}$ the set of isotopy classes of $n$-tangles. A closure of an $n$-tangle $T$, written $T^{\wedge}$, is the link diagram formed by joining the $n$ points at the top of $T$ to those at the bottom without further crossings. For an $m$-braid $b(m \geq n)$, the $n$-closure of $b$ is the $n$-tangle formed by joinig the $i$-th poont $(n+1 \leq i \leq m)$ at the top of $b$ to that at the bottom without further crossings (Figure 16).

As in Theorem 1.1.1, we have the following.

Theorem 6.1.2. Every n-tangle is isotopic to the n-closure of a braid.

For two $n$-tangles $T$ and $S$, we define the composite tangle $T S$ by connecting the points at the bottom of $T$ to those at the top of $S$ as in Figure 17. The set $R_{n}$ together with the above composition law is a semigroup called the $n$-tangle semigroup. An $n$-braid is an $n$-tangle and we regard $B_{n}$ as a subsemigroup of 


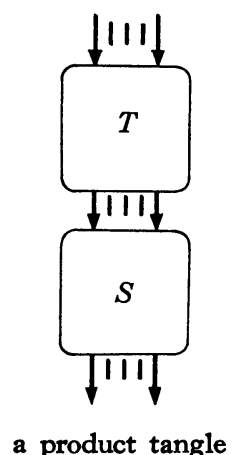

Figure 17

$R_{n}$. Let $C R_{n}$ denote the semigroup algebra of $R_{n}$ called the $n$-tangle algebra.

Definition 6.1.3. For a positive integer $r$, the $r$-parallel version $T^{(r)}$ of an $n$-tangle $T$ is obtained by rep'acing each crossings of $T$ as in Figure 1.

Proposition 6.1.4. Let $T$ and $S$ be $n$-tangles. Their $r$-parallel versions $T^{(r)}$ and $S^{(r)}$ are isotopic if $T$ and $S$ are isotopic.

This proposition is proved by an argument analogous to the proof of Theorem 1.2.2. We use the Reidemeister moves instead of the relations in Definition 1.1.2.

Let $\phi_{n}^{(r)}: C R_{n} \rightarrow C R_{r n}$ be the algebra homomorphism defined by $\phi_{n}^{(r)}(T)=T^{(r)}$. Let $\iota_{k} \sim: B_{r} \rightarrow C B_{r n}$ be the composition of $\iota_{k}$ defined by (1.4.2) and the natural inclusion $B_{r n} \rightarrow C R_{r n}$. Let $\theta: R_{n} \rightarrow S_{n}$ be the mapping such that, for $T \in R_{n}$, the $i$-th point at the top of $T$ is joined to the $\theta(T)(i)$-th point at the bottom by a curve of $T$. Then $\theta$ is a semigroup homomorphism.

Lemma 6.1.5. For $T \in R_{n}$ and $b \in B_{r}$,

$$
\phi_{n}^{(r)}(T) \iota_{k} \sim\left(b^{\prime}\right)=\iota_{\theta(T)(K)} \sim\left(b^{\prime}\right) \phi_{n}^{(r)}(T) \quad(1 \leq k \leq n) .
$$

Proof. Let $(b, m) \in B$ such that the $n$-closure of $b$ is isotopic to $T$. Then $\phi_{n}^{(r)}(T)$ is isotopic to the $r n$-closures of $\phi_{m}^{(r)}(b)$. Hence the statement of the lemma follows from Lemma 1.4.3 and the fact that the $r n$-closures of $\left(\phi_{m}^{(r)}(b) \iota_{k}(c)\right)$ and $\left(\iota_{k}(c) \phi_{m}^{(r)}(b)\right)$ are isotopic for $c \in B_{r}$ if $n+1 \leq k \leq m$.

In the following of this section let $X$ be the one-variable Jones polynomial (two-variable Jones polynomial, the Kauffman polynomial respectively). We fix the complex parameters of the invariant $X$ so that $X$ is of trace type and the corresponding coefficients $a_{n, i}(V)$ in (4.1.9) $\left(a_{\lambda}(P)\right.$ in Theorem 3.4, $a_{i, \mu}(F)$ in Theorem 5.1.5 respectively) are all non-zero. Let $X_{n}$ be the linear function on 
the associated algebra $A_{n}(X)$ defined by $X\left((b, n)^{\wedge}\right)=X_{n}\left(p_{n}(b)\right)$ for $b \in B_{n}$. Then from the defining relation of these invariants, we have the following.

Proposition 6.1.6. There is an algebra homomorphism. $\Xi_{n}: C R_{n} \rightarrow A_{n}(X)$ such that $X\left(T^{\wedge}\right)=X_{n}\left(\Xi_{n}(T)\right)$ for $T \in R_{n}$.

Proof. We prove this in the case when $X$ is the Kauffman polynomial $F(\cdot)$ $(a, x)$. The proofs for one and two-variable Jones polynomials are similar. Let $C_{m}(m \in N)$ be the associated algebras of the Kauffman polynomial (Section 5.1). Let $F_{m}: C_{m} \rightarrow C$ be the mapping defined by $F_{m}\left(p_{m}(b)\right)=F\left(b^{\wedge}\right)$ for $b \in B_{m}$. We first define a linear mapping $\Phi_{m}: C_{m} \rightarrow C_{m-1}$. Let $(\cdot, \cdot)_{m}$ be the symmetric bilinear form on $C_{m}$ defined by using $F_{m}: C_{m} \rightarrow C$ as follows:

$$
\left(y_{1}, y_{2}\right)_{m}=F_{m}\left(y_{1} y_{2}\right) \quad \text { for } \quad y_{1}, y_{2} \in C_{m} \text {. }
$$

Since $(\cdot, \cdot)$ is non-degenerate by the assumption of the parameters $(a, x)$ of $F$, we can uniquely define $\Phi_{m}$ so that $\Phi_{m}$ satisfies $\left(y_{1}, \Phi_{m}\left(y_{2}\right)\right)_{m-1}=\left(y_{1}, y_{2}\right)_{m}$ for all $y_{1} \in C_{m-1}$ and $y_{2} \in C_{m}$. By putting $y_{1}=1$, we have $F_{m}\left(y_{2}\right)=F_{m-1}\left(\Phi_{m}\left(y_{2}\right)\right)$. By using the defining relations (5.1.1) of $C_{m}$, any element $y \in C_{m}$ can be written as $y=z_{1}+z_{2} z_{m-1} z_{3}+z_{4} \tau_{m-1} z_{5}$ for some $z_{1}, \cdots, z_{5} \in C_{m-1}$. By using Definition 1.1.10, we know that $\Phi_{m}(y)=\left(1-\left(a-a^{-1}\right) / x\right) y_{1}+y_{2} z_{2}+a y_{3} z_{3}$. Let $\lambda_{r}: C_{m} \rightarrow$ $C_{m+r}$ and $\lambda_{r}^{\prime}: B_{m} \rightarrow B_{m+r}$ be the algebra homomorphisms defined by $\lambda_{r}\left(\varepsilon_{i}\right)=\varepsilon_{i+r}$, $\lambda_{r}\left(\tau_{i}^{ \pm 1}\right)=\tau_{i+r}^{ \pm 1}$ and $\lambda_{r}^{\prime}\left(\sigma_{i}^{ \pm 1}\right)=\sigma_{i+r}^{ \pm 1}$ respectively. Then

$$
\Phi_{m+r}\left(\lambda_{r}(y)\right)=\lambda_{r}\left(\Phi_{m}(y)\right) \quad \text { for } \quad y \in C_{m},
$$

and

$$
p_{m+r}\left(\lambda_{r}^{\prime}(b)\right)=\lambda_{r}\left(p_{m}(b)\right) \quad \text { for } \quad b \in B_{m} .
$$

Hence we have

$$
\Phi_{m}\left(y_{1} y_{2}\right)=\Phi_{m}\left(y_{1}\right) y_{2} \quad \text { for } \quad y_{1} \in C_{m} \quad \text { and } \quad y_{2} \in C_{m-1} \text {, }
$$

where $p_{m}: C B_{m} \rightarrow C_{m}$ is the projection defined in Section 5.1. Let $T$ be an $n$ angle. Then there is $(b, m) \in B$ whose $n$-closure is isotopic to $T$. Let

$$
\Xi_{n}(T)=\Phi_{n+1}\left(\cdots\left(\Phi_{m-1}\left(\Phi_{m}\left(p_{m}(b)\right)\right)\right) \cdots\right) .
$$

From now on we prove that $\Xi_{n}(T S)=\Xi_{n}(T) \Xi_{n}(S)$ for two $n$-tangles $T$ and $S$. Let $(b, m),\left(b^{\prime}, m^{\prime}\right) \in B$ whose $n$-closures are isotopic to $T$ and $S$ respectively. Then the product tangle $T S$ is isotopic to the $n$-closure of $\left(\delta^{-1} \lambda_{m^{\prime}-n}(b) \delta b^{\prime}\right.$, $\left.m+m^{\prime}-n\right)$ where $\delta=\sigma\left(m^{\prime}-n, 1, m^{\prime}-1, n\right)$ (see (4.4.17)). By using (6.1.8)(6.1.11), for $y \in C_{n}$, we have 


$$
\begin{aligned}
F_{n} & \left(y \Xi_{n}(T S)\right) \\
& \left.=F_{n}\left(y \Phi_{n+1}\left(\cdots\left(\Phi_{m+m^{\prime}-n}\left(p_{m+m^{\prime}-n}\left(\delta^{-1} \lambda_{m^{\prime}-n}^{\prime}(b) \delta b^{\prime}\right)\right)\right)\right) \cdots\right)\right) \\
& =F_{n}\left(y \Phi_{n+1}\left(\cdots\left(\Phi_{m^{\prime}}\left(\delta^{-1} \Phi_{m^{\prime}+1}\left(\cdots\left(\Phi_{m+m^{\prime}-n}\left(p_{m+m^{\prime}-n}\left(\lambda_{m^{\prime}-n}^{\prime}(b)\right)\right)\right) \cdots\right) \delta p_{m^{\prime}}\left(b^{\prime}\right)\right)\right) \cdots\right)\right) \\
& =F_{n}\left(y \Phi_{n+1}\left(\cdots\left(\Phi_{m^{\prime}}\left(\delta^{-1} \lambda_{m^{\prime}-n}\left(\Phi_{n+1}\left(\cdots\left(\Phi_{m}\left(p_{m}(b)\right)\right) \cdots\right)\right) \delta p_{m^{\prime}}\left(b^{\prime}\right)\right)\right) \cdots\right)\right) \\
& =F_{n}\left(y \Phi_{n+1}\left(\cdots\left(\Phi_{m^{\prime}}\left(\Phi_{n+1}\left(\cdots\left(\Phi_{m}\left(p_{m}(b)\right)\right) \cdots\right) p_{m^{\prime}}\left(b^{\prime}\right)\right)\right) \cdots\right)\right) \\
& =F_{n}\left(y \Phi _ { n + 1 } \left(\cdots \left(\Phi_{m^{\prime}}\left(\Phi_{n+1}\left(\cdots\left(\Phi_{m}\left(p_{m}(b)\right)\right) \cdots\right) \Phi_{n+1}\left(\cdots\left(\Phi_{m^{\prime}}\left(p_{m^{\prime}}\left(b^{\prime}\right)\right)\right) \cdots\right)\right)\right.\right.\right. \\
& =F_{n}\left(y \Xi_{n}(T) \Xi_{n}(S)\right) .
\end{aligned}
$$

Hence we get $\Xi_{n}(T S)=\Xi_{n}(T) \Xi_{n}(S)$.

For $\mu \in A_{r n}(X)^{\wedge}$ (respectively $\left.\nu \in A_{r}(X)^{\wedge}\right)$, let $\left(\rho_{\mu}, V_{\mu}\right)$ (respectively $\left(\rho_{\nu}, V_{\nu}\right)$ ) be the corresponding representation of $A_{r n}(X)$ (respectively $A_{r}(X)$ ) and $\chi_{\mu}$ (respectively $\chi_{\nu}$ ) its character. We regard $B_{r}^{n}$ as a subgroup of $B_{r n}$ as in Section 1.4. Then $V_{\mu}$ can be regarded as a $B_{r}^{n}$-module. For $\nu \in A_{r}(X)^{\wedge}$, let $V_{\mu, \nu, \cdots, \nu}$ be the $\left(\rho_{\nu} \circ p_{r}\right)^{\otimes n}$-isotypic subspace of $V_{\mu}$. Then $V_{\mu, \nu, \cdots, \nu}$ is invariant relative to the action of $\phi_{n}^{(r)}\left(R_{n}\right)$. Let $\left(\rho_{\mu, \nu}, V_{\mu, \nu, \cdots, \nu}\right)$ denote the representation $\left.\rho_{\mu} \circ \Xi_{r n} \phi_{n}^{(r)}\right|_{\nu \mu, \nu}$ of $R_{r n}$. From Proposition 1.3.4, we know that there is a representation $\pi_{\mu, \nu}$ of $R_{n}$ such that $\rho_{\mu, \nu} \simeq\left(\rho_{\nu}^{\otimes n}\right)^{\sim} \otimes \pi_{\mu, \nu}$, where $\left(\rho_{\nu}^{\otimes n}\right)^{\sim}$ is the representation of $B_{r}^{n} \searrow R_{n}$ coming from $\rho_{\nu}^{\otimes n}$ as in Proposition 1.3.4. Let $\omega_{\mu, \nu}$ be the character of $\pi_{\mu, \nu}$. Then the argument proving Theorem 1.4.10 implies the next one.

Theorem 6.1.12. Let $T$ be an n-tangle whose closure is a knot. Then we have the following.

(i) $\chi_{\mu}\left(\Xi_{r n}\left(\phi_{n}^{(r)}(T)\right)\right)=\sum_{\nu \in A_{r}(Z) \wedge} \chi_{\nu}(1) \omega_{\mu, \nu}(T)$,

(ii) $X^{(r)}\left(T^{\wedge}\right)=\sum_{\mu \in A_{r n}(X) \wedge}\left(\sum_{\nu \in A_{r}(X) \wedge} a_{\mu}(X) \chi_{\nu}(1) \omega_{\mu, \nu}(T)\right)$.

\subsection{Mutation.}

In this section, let $X$ be the one-variable Jones polynomial $V$ (the twovariable Jones polynomial $P$, the Kauffman polynomial $F$ respectively).

Definition 6.2.1. For $T$ in the $n$-tangle algebra $R_{n}$, we define three nontrivial involutions $\gamma_{1}, \gamma_{2}$ and $\gamma_{3}$ (see Figure 18). Let $\gamma_{1}$ be the involution defined by $\gamma_{1} T=g T g^{-1}$ where $g=\sigma(1, n) \sigma(1, n-2) \cdots \sigma(1,1)$ and $\sigma(i, j)=\sigma_{i} \sigma_{i-1} \cdots \sigma_{j}$ $(i \leq j)$. Let $D_{T}$ be a rectangular diagram of $T$ and $H$ the half-turn of $D_{T}$ about the center of $D_{T}$. Put $T^{\prime}=H\left(D_{T}\right)$. Let $\gamma_{2} T$ be the element of $R_{n}$ which is the isotopy class of the tangle obtained by inverting the orientations of all the strings of $T^{\prime}$. Let $\gamma_{3}$ denote the composition of $\gamma_{1}$ and $\gamma_{2}$.

The involution $\gamma_{1}$ induces an automorphism of the 2-tangle algebra $C R_{2}$, and $\gamma_{2}$ and $\gamma_{3}$ induce anti-automorphisms of $C R_{2}$. Hence they induce (anti-) automorphisms of the subalgebra $\Xi_{r n}\left(\phi_{n}^{(r)}\left(\boldsymbol{C} R_{n}\right)\right)$ of $A_{r n}(X)$, where $\Xi_{r n}: C R_{r n} \rightarrow$ $A_{r n}(X)$ is the algebra homomorphism introduced in Proposition 6.1.6. 


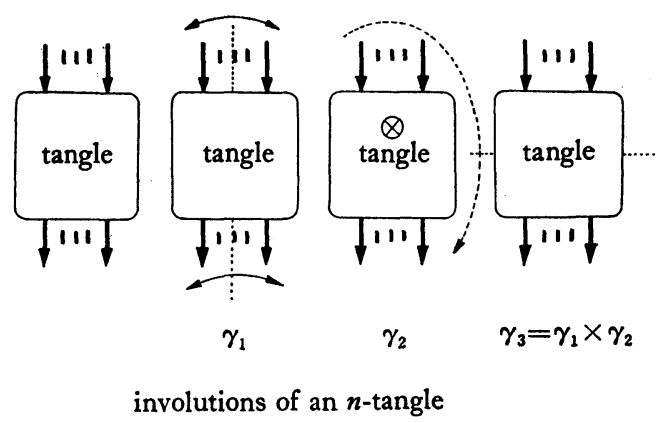

Figure 18

Proposition 6.2.2. Let $\mu \in A_{r n}(X)^{\wedge}$ and $\nu \in A_{r}(X)^{\wedge}$. If the degree (i.e. the dimension of the representation space) of the representation $\pi_{\mu, \nu}$ (introduced at the end of the last section) is equal to zero or one, then we have

$$
\pi_{\mu, \nu}(T)=\pi_{\mu, \nu}\left(\gamma_{i} T\right) \quad \text { for } \quad T \in R_{n}, 1 \leq i \leq 3 .
$$

Proof. Lemma 6.2.9, which will be gieven at the end of this section, shows that the actions of $\gamma_{i}(1 \leq i \leq 3)$ induce $C$-algebra (anti-)automorphisms of $\pi_{\mu, \nu}$ $\left(C R_{2}\right)$. But $\pi_{\mu, \nu}\left(C R_{2}\right)$ is isomorphic to $C$ or $\{0\}$ by the assumption. Hence their actions must be trivial ones.

Definition 6.2.3 (see, e.g. [8]). Two links $K$ and $K^{\prime}$ are called mutant if there are two 2-atngles $T, S$ and an involution $\gamma_{i}(i=1,2$ or 3$)$ of $S$ such that $K$ and $K^{\prime}$ are equivalent to $T S^{\wedge}$ and $\left(T \gamma_{i} S\right)^{\wedge}$ respectively.

The following theorem is the main result of this section.

Theorem 6.2.4. Let $K$ and $K^{\prime}$ be mutant knots. Then, for a positive integer $r$ and $\nu \in A_{r}(X)^{\wedge}, X^{(r, \nu)}(K)=X^{(r, \nu)}\left(K^{\prime}\right)$ if the degrees of the representations $\pi_{\mu, \nu}$ $\left(\mu \in A_{2 r}(X)\right)$ are all equal to zero or one.

Proof. Let $T$ and $S$ be the tangles and $\gamma$ an involution of $S$ such that $K$ and $K^{\prime}$ are equivalent to $T S^{\wedge}$ and $(T \gamma S)^{\wedge}$ respectively. Theorem 6.1.9 implies that

$$
\begin{aligned}
& X^{(r, \nu)}(K)=\chi_{\nu}(1) \sum_{\mu \in A_{2,}(X) \wedge} a_{n}(X) \operatorname{trace}\left(\pi_{\mu, \nu}(T) \pi_{\mu, \nu}(S)\right), \\
& X^{(r, \nu)}\left(K^{\prime}\right)=\chi_{\nu}(1) \sum_{\mu \in A_{r_{2}(x) \wedge}} a_{\mu}(X) \operatorname{trace}\left(\pi_{\mu, \nu}(T) \pi_{\mu, \nu}(\gamma S)\right) .
\end{aligned}
$$

Hence the statement of the theorem follows from Proposition 6.2.2.

Corollary 6.2.5. Let $K$ and $K^{\prime}$ be mutant knots. Then we have

$$
V^{(r, v)}(K)=V^{(r, v)}\left(K^{\prime}\right),
$$


(ii) $([23],[25]) \quad V^{(r)}(K)=V^{(r)}\left(K^{\prime}\right)$,

(iii) $\quad P^{(r, \mu)}(K)=P^{(r, \mu)}\left(K^{\prime}\right)$ if the partition $\mu$ of $r$ is equal to $(r)$ or $\left(1^{r}\right)$,

$$
\begin{aligned}
& P^{(2)}(K)=P^{(2)}\left(K^{\prime}\right), \\
& F^{(2)}(K)=F^{(2)}\left(K^{\prime}\right) .
\end{aligned}
$$

Proof. Recall that the degree of $\pi_{\mu, \nu}$ is equal to the multiplicities of $\rho_{\nu}^{\otimes 2}$ in $\rho_{\mu}$ for $\mu \in A_{2 r}(X)^{\wedge}$ and $\nu \in A_{r}(X)$. By Remark 3.8, the above multiplicities may be calculated by the Littlewood-Richardson rule in the case that $X$ is the Jones polynomial $V$ or the two-variable Jones polynomial $P$. This rule shows that the degree of the representations $\pi_{\mu, \nu}$ for the case (i)-(iv) are all equal to 1 or 0 . To prove (v), we show that the degree of $\pi_{\mu, \nu}$ is equal to 1 or 0 for $\mu \in A_{4}(F)^{\wedge}$ and $\nu \in A_{2}(F)^{\wedge}$. This can be checked by using the realizations of irreducible representations of $A_{4}(F)$ given in [2].

Theorem 6.2.6. Let $K$ and $K^{\prime}$ be mutant knots, i.e. there are tangles $T$ and $S$ and an involution $\gamma$ of $S$ such that $K$ and $K^{\prime}$ are equivalent to $T S^{\wedge}$ and $(T \gamma S)^{\wedge}$. Put $\lambda_{0}=(321) \in \Lambda(6)$ and $V_{n}=(21) \in \Lambda(3)$. Then, with the notations in Theorem 6.1.12 we have $P^{(3)}(K) \neq P^{(3)}\left(K^{\prime}\right)$ iff $a_{\lambda_{0}}(P) \neq 0$ and $\omega_{\lambda_{0}, \nu_{0}}(T S) \neq \omega_{\lambda_{0}, \nu_{0}}(T \gamma S)$.

Proof. From Theorem 1.5.1 and Corollary 6.2.5 we have $P^{(3)}(K) \neq P^{(3)}\left(K^{\prime}\right)$ iff $P^{\left(3, v_{0}\right)}(K) \neq P^{\left(3, v_{0}\right)}\left(K^{\prime}\right)$. But from Littlewood-Richardson rule (Remark 3.8), the degree of $\pi_{\lambda_{0}, v_{0}}$ is equal to 2 and those of $\pi_{\lambda, v_{0}}$ are equal to 1 or 0 if $\lambda \neq \lambda_{0}$. Hence Proposition 6.2.2 implies the statement of the theorem.

EXAmple 6.2.7. We give four mutant knots $K_{1}, K_{2}, K_{3}$ and $K_{4}$ for which the invariants $P^{(3)}\left(K_{i}\right)(1 \leq i \leq 4)$ are all distinct. Let $S_{1}, S_{2}, \sigma$ and $\sigma^{\sim}$ be the tangles as in Figure 19, $S_{3}=S_{1} \sigma S_{3}, S_{4}=S_{1} S_{2}$ and $K_{i}=\left(S_{3} \gamma_{i}\left(S_{4}\right)\right)^{\wedge}(1 \leq i \leq 4$, Figure 19). Let $q$ be an indeterminate and $H_{6}^{\cdot}$ the $\boldsymbol{Z}\left[q^{1 / 2}, q^{-1 / 2}\right]$-algebra with unit defined by the relations (3.2). Let $\Phi_{m}: H_{m}{ }^{\cdot} \rightarrow H_{m-\mathrm{I}} \cdot$ be the mapping defined as in the proof of Proposition 6.1.6. Then, from the definition of the two-variable Jones polynomial, we know that the image $\Phi_{m}\left(H_{m}{ }^{*}\right)$ is contained in $H_{m-1} \cdot \otimes_{Z} Z\left[\alpha, \alpha^{-1},\left(\alpha q^{1 / 2}-\alpha^{-1} q^{-1 / 2}\right)^{-1}\right]$. Hence $\Xi_{n}\left(R_{n}\right)(n=3,6)$ are contained in $H_{n} \otimes_{Z} Z\left[\alpha, \alpha^{-1},\left(\alpha q^{-1}-\alpha^{-1} q^{-1 / 2}\right)^{-1}\right]$ by (6.1.11). On the other hand, Naruse and Gyoja constructed $W$-graphs [18] of all the irreducible repre sentations of $H_{6}$ (Figure 8). This means that, for each irreducible representation, they give a basis of the representation space and representation matrices of generators of $H_{6}$ with respect to the basis. The entries of the above representation matrices of the generators $T_{i} \in H_{6}{ }^{\circ}(1 \leq i \unlhd 5)$ are all contained in $\boldsymbol{Z}\left[q^{1 / 2}, q^{-1 / 2}\right]$. This implies the following two facts. Let $\mathcal{R}=\boldsymbol{Z}\left[q^{1 / 2}, q^{-1 / 2}, \alpha, \alpha^{-1},\left(\alpha q^{1 / 2}-\right.\right.$ $\left.\left.\alpha^{-1} q^{-1 / 2}\right)^{-1}\right]$. The first fact is that the composition of $\Xi_{6}$ and the above representations of $H_{6}^{*}$ define irreducible representations of $R_{6}$ over $\mathscr{R}$. The second fact is that the $\nu_{0}$-isotypic subspace $V_{\lambda_{0}, \nu_{0}}$ of the representation space 

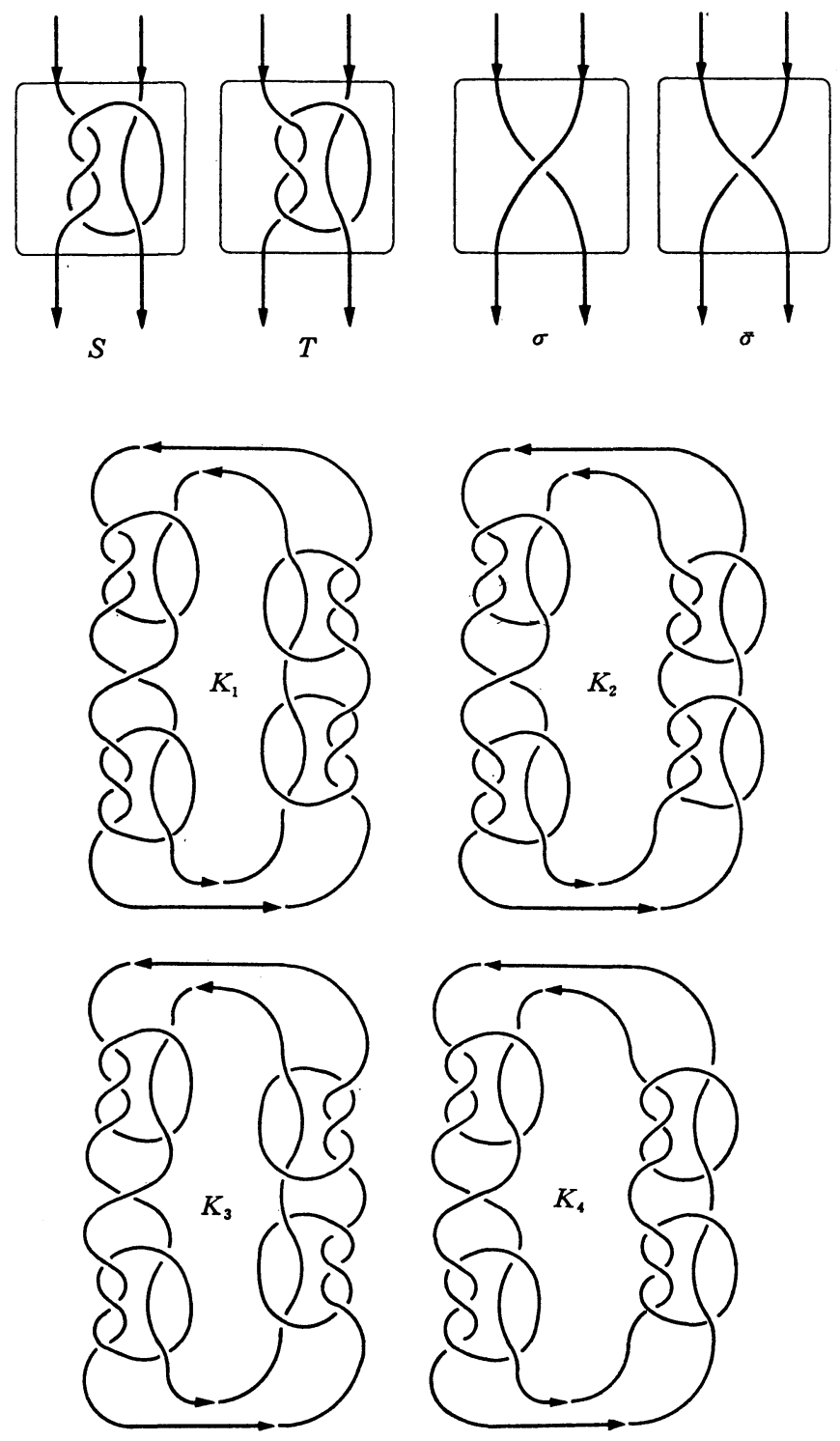

Figure 19

$V_{\lambda_{0}}$ of $\rho_{\lambda_{0}}$ is defined over $\mathcal{R}$. Hence we can define the reduction modulo $p$ of the representation $\pi_{\lambda_{0}, v_{0}}$ of $R_{2}$. This is defined over $\boldsymbol{F}_{p}\left[q^{1 / 2}, q^{-1 / 2}, \alpha, \alpha^{-1}\right.$, $\left.\left(\alpha q^{1 / 2}-\alpha^{-1} q^{-1 / 2}\right)^{-1}\right]$, where $\boldsymbol{F}_{p}$ is the finite field of prime order $p$. In the following, we put $p=23, \alpha=1$ and $q=2$. With these parameters, we know that $\alpha q^{1 / 2}-\alpha^{-1} q^{-1 / 2} \neq 0$ and $a_{\lambda_{0}}(P) \neq 0(\bmod 23)$ from the explicit formula for $a_{\lambda_{0}}(P)$ given in [10] or [15]. Therefore we may use Theorem 6.2.7. We calculate the elements $\Xi_{6}\left(\phi_{2}^{(3)}\left(S_{1}\right)\right), \Xi_{6}\left(\phi_{2}^{(3)}\left(S_{2}\right)\right), \Xi_{6}\left(\phi_{2}^{(3)}(\sigma)\right)$ and $\Xi_{6}\left(\phi_{2}^{(3)}\left(\sigma^{\sim}\right)\right)$ of $\left(H_{6}^{\cdot} \otimes_{Z}\right.$ 
$\left.\boldsymbol{F}_{23}\right)_{\alpha=1, q=2}$ with the aid of computers (Turbo PASCAL on MS-DOS for PC-9801 (NEC) and AOS/VS PASCAL on Eclipse MV-2000DC (DG)). Their images under $\pi_{\lambda_{0}, \nu_{0}}$ with respect to the explicit basis mentioned above are

$$
\begin{array}{ll}
\pi_{\lambda_{0}, \nu_{0}}\left(\phi_{2}^{(y)}\left(S_{1}\right)\right) \equiv\left[\begin{array}{cc}
9 & 1 \\
7 & 16
\end{array}\right], & \pi_{\lambda_{0}, \nu_{0}}\left(\phi_{2}^{(3)}\left(S_{2}\right)\right) \equiv\left[\begin{array}{cc}
8 & 19 \\
0 & 5
\end{array}\right], \\
\pi_{\lambda_{0}, \nu_{0}}\left(\phi_{2}^{(3)}(\sigma)\right) \equiv\left[\begin{array}{cc}
0 & 19 \\
19 & 0
\end{array}\right], & \pi_{\lambda_{0}, \nu_{0}}\left(\phi_{2}^{(3)}\left(\sigma^{\sim}\right)\right) \equiv\left[\begin{array}{cc}
0 & 21 \\
21 & 0
\end{array}\right](\bmod 23) .
\end{array}
$$

Hence we have

$$
\begin{aligned}
& \pi_{\lambda_{0}, \nu_{0}}\left(\left.\phi\right|^{(3)}\left(S_{3}\right)\right)=\pi_{\Lambda_{0}, \nu_{0}}\left(\phi_{2}^{(3)}\left(S_{1} \sigma S_{2}\right)\right) \equiv\left[\begin{array}{cc}
14 & 20 \\
17 & 1
\end{array}\right], \\
& \pi_{\lambda_{0}, \nu_{0}}\left(\phi_{2}^{(3)}\left(S_{4}\right)\right)=\pi_{\lambda_{0}, \nu_{0}}\left(\phi_{2}^{(3)}\left(S_{1} S_{2}\right)\right) \equiv\left[\begin{array}{cc}
3 & 15 \\
10 & 6
\end{array}\right], \\
& \pi_{\lambda_{0}, \nu_{0}}\left(\phi_{2}^{(3)}\left(\gamma_{1} S_{4}\right)\right)=\pi_{\lambda_{0}, \nu_{0}}\left(\phi_{2}^{(3)}\left(\sigma S_{1} S_{2} \sigma^{\sim}\right)\right) \equiv\left[\begin{array}{cc}
6 & 10 \\
15 & 3
\end{array}\right], \\
& \pi_{\lambda_{0} \nu_{0}}\left(\phi_{2}^{(3)}\left(\gamma_{2} S_{4}\right)\right)=\pi_{\lambda_{0}, \nu_{0}}\left(\phi_{2}^{(3)}\left(S_{2} S_{1}\right)\right) \equiv\left[\begin{array}{ll}
21 & 13 \\
12 & 11
\end{array}\right], \\
& \pi_{\lambda_{0}, \nu_{0}}\left(\phi_{2}^{(3)}\left(\gamma_{3} S_{4}\right)\right)=\pi_{\lambda_{0}, \nu_{0}}\left(\phi_{2}^{(3)}\left(\sigma S_{2} S_{1} \sigma^{\sim}\right)\right) \equiv\left[\begin{array}{ll}
11 & 12 \\
13 & 21
\end{array}\right](\bmod 23) .
\end{aligned}
$$

Thus we have $\omega_{\lambda_{0}, \nu_{0}}\left(\phi_{2}^{(3)}\left(S_{3} S_{4}\right)\right) \equiv 20, \omega_{\lambda_{0}, \nu_{0}}\left(\phi_{2}^{(3)}\left(S_{3} \gamma_{1} S_{4}\right)\right) \equiv 5, \omega_{\lambda_{0}, \nu_{0}}\left(\phi_{2}^{(3)}\left(S_{3} \gamma_{2} S_{4}\right)\right)$ $\equiv 7$ and $\omega_{\lambda_{0}, \nu_{0}}\left(\phi_{2}^{(3)}\left(S_{3} \gamma_{3} S_{4}\right)\right) \equiv 18(\bmod 23)$. Hence the invariants $P^{(3)}\left(K_{i}\right)$ $(1 \leq i \leq 4)$ are all distinct by Theorem 6.2 .6 and so any two of the knots $K_{1}, K_{2}, K_{3}$ and $K_{4}$ are not equivalent.

In the rest of this paper, we shall prove the remaining part of the proof of Proposition 6.2.2.

Lemma 6.2.8. Let $(\rho, U)$ be an irreducible representation of $A_{r n}(X)$. Then $\gamma_{i}(i=1,2$ or 3$)$ induces an automorphism (respectively anti-automorphism) of $\operatorname{End}(U)$ defined by ${ }^{\gamma_{i}}(\rho(y))=\rho\left(\gamma_{i} y\right)\left(y \in A_{r n}(X)\right)$.

Proof. $\left.1^{\circ}\right)$ The case $i=1$. For $y \in A_{r n}(X)$, we have $\gamma_{1} y=p_{r n}\left(\phi_{n}^{(r)}(g)\right) y p_{r n}$ $\left(\phi_{n}^{(r)}(g)\right)^{-1}$ where $g=\sigma(1, n) \sigma(1, n-2) \cdots \sigma(1,1), \sigma(i, j)=\sigma_{i} \sigma_{i+1} \cdots \sigma_{j}(i \leq j)$ and $p_{r n}$ be the projection $\boldsymbol{C} B_{r n} \rightarrow A_{r n}(X)$. Hence $\gamma_{1}$ acts on $\operatorname{End}(U)$ by ${ }^{\gamma_{1}}(\rho(y))=\rho\left(p_{r n}\right.$ $\left(\phi_{n}^{(r)}(g)\right) \rho(y) \rho\left(p_{r n}\left(\phi_{n}^{(r)}(g)\right)\right)^{-1}$.

$2^{\circ}$ ) The case $\gamma=\gamma_{2}$. In this case, the statement of the lemma follows from $1^{\circ}$ ) and $3^{\circ}$ ) since $\gamma_{2}=\gamma_{1}^{\circ} \circ \gamma_{3}$.

$3^{\circ}$ ) The case $\gamma=\gamma_{3}$. We know realizations of every irreducible represen- 
tations of $A_{r n}(X)$ such that $p_{r n}\left(\sigma_{i}\right)(1 \leq i \leq r n-1)$ are represented by symmetric matrices. When $X=V(P, F$ respectively), such constructions are given in [31] ([31], [28] respectively). For a matrix $M$, let ${ }^{t} M$ denote the transposed matrix of $M$. We may assume that ${ }^{t} \rho\left(p_{r n}\left(\sigma_{i}\right)\right)=\rho\left(p_{r n}\left(\sigma_{i}\right)\right)$. Since $\gamma_{3}$ satisfies $\gamma_{3}\left(p_{r n}\left(\sigma_{i}\right)\right)=p_{r n}\left(\sigma_{i}\right)(1 \leq i \leq r n-1)$ and $\gamma_{3}\left(h h^{\prime}\right)=\gamma_{3}\left(h^{\prime}\right) \gamma_{3}(h)$ for $h, h^{\prime} \in A_{r n}$, we have ${ }^{\gamma_{3}} \rho(h)={ }^{t} \rho(h)\left(h \in A_{r n}(X)\right)$. Hence $\gamma_{3}$ acts on $\operatorname{End}(U)$ by the matrix transposition.

Lemma 6.2.9. Let $\left(\pi_{\mu, \nu}, W_{\mu, \nu}\right)\left(\mu \in A_{2 r}(X)^{\wedge}, \nu \in A_{r}(X)^{\wedge}\right)$ be the representation of $C R_{2}$ given at the end of Section 6.1. Then $\gamma_{i}(i=1,2,3)$ induces an (anti-) autooautomorphism of $\pi_{\mu, \nu}\left(\boldsymbol{C R}_{2}\right)$ defined by $\left.{ }^{\gamma_{i}}\left(\pi_{\mu, \nu}(y)\right)=\pi_{\mu, \nu}\left(\gamma_{i} y\right)\right)\left(y \in A_{2 r}(X)\right)$.

Proof. $1^{\circ}$ ) The case $\gamma=\gamma_{1}$. The statement of the lemma follows from $\gamma_{1}\left(\pi_{\mu, \nu}(y)\right)=\pi_{\mu, \nu}(g) \pi_{\mu, \nu}(y) \pi_{\mu, \nu}(g)^{-1}$ where $g=\sigma(1, n-1) \sigma(1, n-2) \cdots \sigma(1,1)$ and $\sigma(i, j)=\sigma_{i} \sigma_{i+1} \cdots \sigma_{j}(i \leq j)$.

$2^{\circ}$ ) The case $\gamma=\gamma_{2}$. In this case, the statement of the lemma follows from $1^{\circ}$ ) and $3^{\circ}$ ) since $\gamma_{2}=\gamma_{1}^{\circ} \circ \gamma_{3}$.

$\left.3^{\circ}\right)$ The case $\gamma=\gamma_{3}$. Let $\left(\rho_{\mu}, V_{\mu}\right)$ be the irreducible representation of $A_{r n}(X)$ parametrized by $\mu$. We shall show that $\pi_{\mu, \nu}\left(\gamma_{3} y\right)={ }^{t}\left(\pi_{\mu, \nu}(y)\right)$ with respect to a basis of $W_{\mu_{\nu}}$. In this proof, we use the following fact.

Lemma 6.2.10. Let $s$ be an indeterminate. Let $y_{1}(s), y_{2}(s) \in M_{m}(\mathcal{K})$ with ${ }^{t} y_{1}(s)=y_{2}(s)$ where $\mathcal{K}$ is the algebraic closure of $\boldsymbol{C}(s)$. Let $U(s)$ be a subspace of $\mathcal{K}^{m}$ such that $y_{1}(s) U(s) \subseteq U(s)$ and ${ }^{t} y_{1}(s) U(s) \subseteq U(s)$. We assume that there is a open set $\mathcal{U}$ of $\boldsymbol{R}$ (the set of real numbers) and a basis $\left\{u_{1}(s), \cdots, u_{m}(s)\right\}$ of $\mathcal{K}^{m}$ such that $\left\{u_{1}(s), \cdots, u_{k}(s)\right\}$ is a basis of $U(s)$ which satisfy the following conditions.

(i) The coordinates of $u_{i}(s)(1 \leq i \leq m)$ have no branch point in $\mathcal{U}$ and they are contained in $\boldsymbol{R}$ for $s \in \mathcal{U}$.

(ii) The vectors $u_{i}(s)(1 \leq i \leq m)$ are linearly independent for $s \in \mathcal{U}$.

(iii) The elements of the matrix of $y_{1}(s)$ with respect to the above basis have no branch point in $\mathcal{U}$.

Then there is a basis of $U(s)$ such that the transpose of the matrix of $\left.y_{1}(s)\right|_{U(s)}$ with respect to this basis is equal to the matrix of $\left.y_{2}(s)\right|_{U(s)}$.

Proof. At first, fix $s \in \mathcal{U}$. By applying the Schmidt's method to $\left\{u_{1}(s), \cdots\right.$, $\left.u_{m}(s)\right\}$, we get an orthonormal basis $\left\{v_{1}(s), \cdots, v_{m}(s)\right\}$ of $\boldsymbol{R}^{m}$ with respect to the standard bilinear form such that $\left\{v_{1}(s), \cdots, v_{k}(s)\right\}$ is an orthonormal basis of $U(s)$. With respect to the basis $\left\{v_{1}(s), \cdots, v_{k}(s)\right\}$, the transpose of the matrix of $y_{1}(s)$ is equal to $y_{2}(s)$. Now we discuss for general $s$. From the above argument, we know that $\left(y_{1}(s)\right)_{i j}=\left(y_{2}(s)\right)_{j i}(1 \leq i, j \leq m)$ for $s \in \mathcal{U}$. But the assumption of the lemma implies that the elements of the matrix of $y_{1}(s)$ with respect to the basis $\left\{v_{1}(s), \cdots, v_{k}(s)\right\}$ are algebraic functions in $s$. Hence the equalities $\left(y_{1}(s)\right)_{i j}=$ $\left(y_{2}(s)\right)_{j i}(1 \leq i, j \leq m)$ also hold when we regards as an indeterminate. 
Now, we return to the proof of Lemma 6.2.9. Let $\left(\rho_{\mu, \nu}, V_{\mu, \nu}\right)$ and $\left(\left(\rho_{\nu}^{\otimes n}\right)^{\sim}\right.$, $\left.V_{\nu}^{\otimes_{n}}\right)$ be the representations of $B_{r}^{n} \backslash R_{n}$ given at the end of Section 6.1. From the definition of $\phi_{n}^{(r)}$, we have

$$
\phi_{n}^{(r)}\left(\gamma_{3} \sigma_{i}\right)=\left(\sigma_{r i-r+1} \sigma_{r i-r+2} \cdots \sigma_{r i-1}\right)^{-r}\left(\sigma_{r i+1} \sigma_{r i+2} \cdots \sigma_{r i+r-1}\right)^{r} \gamma_{3} \phi_{n}^{(r)}\left(\sigma_{i}\right) .
$$

We also have $\phi_{n}^{(r)}\left(\gamma_{3} y\right)=c_{y} \gamma_{3} \phi_{n}^{(r)}(y)$ where $c_{y}$ is a product of some $\left(\sigma_{r i+1} \sigma_{r i+2} \cdots\right.$ $\left.\sigma_{r i+r-1}\right)^{ \pm r}(1 \leq i \leq n-1)$. Note that the exponent sum of $c_{y}$ is equal to zero. The elements $\rho_{\mu}\left(\left(\sigma_{r i+1} \sigma_{r i+2} \cdots \sigma_{r i+r-1}\right)^{-r}\right)(1 \leq i \leq n-1)$ act on $V_{\mu, \nu}$ by the same scalar $\rho_{\nu}\left(\left(\sigma_{1} \sigma_{2} \cdots \sigma_{r-1}\right)^{-r}\right)$ since $\left(\sigma_{1} \sigma_{2} \cdots \sigma_{r-1}\right)^{-r}$ are contained in the center of the subgroup $B_{r}$. Since the exponent sum of $c_{y}$ is equal to zero, we have $\rho_{\mu, \nu}\left(c_{y}\right)=1$. Recall that $V_{\mu, \nu}$ is the $\rho_{\nu}^{\otimes n}$-isotypic subspace of $V_{\mu}$ as a $\boldsymbol{C} B_{r}^{n}$-module and the representation $\rho_{\mu, \nu}$ of $R_{n}$ is given by restricting $\rho_{\mu} \circ \Xi_{r n}^{\circ} \phi_{n}^{(r)}$ to $V_{\mu, \nu}$. The constructions of the irreducible representations of $A_{k}(X)(k \in N)$ refered in the proof of Lemma 6.2.8 imply that $V_{\mu}, V_{\mu, \nu}$ and $\rho_{\mu, \nu}(y)\left(y \in R_{n}\right)$ satisfy the assumption of Lemma 6.2 .10 for $s=q^{1 / 2}$. Therefore we have $\rho_{\mu, \nu}\left(\gamma_{3} y\right)={ }^{t}\left(\rho_{\mu, \nu}(y)\right)(1 \leq i \leq$ $n-1)$ with respect to some basis of $V_{\mu, \nu}$. Let $\theta: R_{n} \rightarrow S_{n}$ be the mapping defined just before Lemma 6.1.5. The representation $\left(\rho_{\nu}^{\otimes n}\right)^{\sim}(y)\left(y \in R_{n}\right)$ gives a parmutation of a basis of $V_{\nu}^{\otimes_{n}}$. Hence $\left(\rho_{\nu}^{\otimes_{n}}\right)^{\sim}$ containes the trivial representation of $R_{n}$ with positive multiplicities. This implies that $\rho_{\mu, \nu}$ containes the representation $\pi_{\mu, \nu}$ with positive multiplicities since $\rho_{\mu, \nu}=\left(\rho_{\nu}^{\otimes_{n}}\right)^{\sim} \otimes \pi_{\mu, \nu}$. Let $U_{\mu, \nu}$ be a $R_{n}$-invariant subspace of $V_{\mu}$ where the representation $\pi_{\mu, \nu} \cdot$ of $R_{n}$ on $U_{\mu, \nu}$ is equivalent to $\pi_{\mu, \nu}$. We identify $\left(\pi_{\mu, \nu}, U_{\mu, \nu}\right)$ and $\left(\pi_{\mu, \nu}, W_{\mu, \nu}\right)$. As in the case of $\rho_{\mu, \nu}$, we can show that there is a basis of $W_{\mu, \nu}$ such that $\pi_{\mu, \nu}\left(\gamma_{3} y\right)={ }^{t}\left(\pi_{\mu, \nu}(y)\right)$ for $y \in C R_{n}$. This is what we wanted to show.

\section{References}

[1] J.S. Birman: Braids, Links, and Mapping Class Groups, Annals of Math. Studies 82, Princeton, 1974.

[2] J.S. Birman and H. Wenzl: Braids, link polynomials and a new algebra, preprint.

[3] N. Bourbaki: Groupes et algebre de Lie, Chapt. IV, V, VI, Hermann, Paris, 1968.

[4] G. Burde and H. Zieschang: Knots, Walter de Gruyter, 1985.

[5] C.W. Curtis, N. Iwahori and R. Kilmoyer: Hecke algebras and characters of parabolic type of finite groups with (B,N)-pairs, I.H.E.S. Publ. Math. 40 (1972), 81-116.

[6] C.W. Curtis and I. Reiner: Representation theory of finite groups and associative algebras, Interscience Publishers, New York 1962.

[7] P. Freyd, D. Yetter, J. Hoste, W.B.R. Lickorish, K. Millet and A. Ocneanu: $A$ new polynomial invariant of knots and links, Bull. Amer. Math. Soc. 12 (1985), 239-246.

[8] C.A. Giller: A family of links and the Conway calculus, Trans. Amer. Math. Soc. 270 (1982), 75-109. 
[9] A. Gyoja: On the existence of a W-graph for an irreducible representation of a Coxeter group, Journal of Algebra 86 (1984), 422-438.

[10] A. Gyoja: Topological invariants of links and representations of Hecke algebras II, preprint, Osaka University.

[11] A. Gyoja and K. Uno: On the semisimplicity of Hecke algebras, J. Math. Soc. Japan 41 (1989), 75-79.

[12] P.N. Hoefsmit: Representations of Hecke algebras of finite groups with BN-pairs of classical type, thesis, The University of British Columbia.

[13] G. James and A. Kerber: The representation theory of the symmetric group, Encyclopedia of math. and its applications 16, Addison-Wesley, 1981.

[14] V.F.R. Jones: A polynomial invariant for knots via von Neumann algebras, Bull. Amer. Math. Soc. 12 (1985), 103-111.

[15] V.F.R. Jones: Hecke algebra representations of braid groups and link polynomials, Ann. Math. 126 (1987), 335-388.

[16] L.H. Kauffman: State models and the Jones polynomial, Topology 26 (1987), 395-407.

[17] L.H. Kauffman: An invariant of regular isotopy, to appear.

[18] D. Kazhdan and G. Lusztig: Representations of Coxeter groups and Hecke algebras, Invent. Math. 53 (1979), 165-184.

[19] A. Kerber: Representations of permutation groups I, Lecture Note in Math. 240, Springer, Berlin, 1971.

[20] D.E. Knuth: The Art of Computer Programming (2nd ed.), Vol. 1, Fundamental Algorithms, Addison-Wesley, 1973.

[21] A. Lascoux and M.P. Schutzenberger: Polynomes de Kazhdan \& Lusztig pour les grassmanniennes, Young tableaux and Schur functions in algebra and geometry (Torun, 1980), pp. 249-266, Asterisque, 87-88, Soc. Math. France, Paris, 1981.

[22] W.B.R. Lickorish: A relationship between link polynomials, Math. Proc. Camb. Philos. Soc. 100 (1986), 109-112.

[23] W.B.R. Lickorish and A.S. Lipson: Polynomials of 2-cable-like links, preprint, Camblidge University, 1986.

[24] H.R. Morton and H.B. Short: The 2-variable polynomial of cable knots, Math. Proc. Camb. Philos. Soc., to appear.

[25] H.R. Morton and P. Traczyk: The Jones polynomial of satellite links around mutants, preprint.

[26] J. Murakami: The Kauffman polynomial of links and representation theory, Osaka J. Math. 24 (1987), 745-758.

[27] J. Murakami: On the Jones invariant of parallel links and linear representations of braid groups, unpublished manuscript.

[28] J. Murakami: The representations of Brauer's centralizer algebra and the Kauffman polynomial of links, in preparation.

[29] J.H. Przytycki and P. Traczyk: Invariants of links of Conway type, Kobe J. Math. 4 (1988), 115-139.

[30] D. Rolfsen: Knots and links. Publish or Perish Inc., 1976.

[31] H. Wenzl: Representations of Hecke algebras and subfactors, Ph.D. Thesis, University of Pensylvania.

[32] H. Wenzl: On the structure of Brauer's centralizer algebras, preprint, University of Calfornia, Berkeley. 
[33] J.H.C. Whitehead: On doubled knots, J. London Math. Soc. 12 (1937), 63-71.

[34] S. Yamada: On the 2-variable Jones polynomial of satellite links, Topology and Computer Science, 295-300, Kinokuniya, Tokyo 1987.

[35] S. Yamada: Some regular isotopy invariants and a link invariant operator, to appear in Topology.

Department of Mathematics Osaka University

Toyonaka, Osaka 560

Japan 
